\title{
Fenômeno de bifurcação no problema de Yamabe sobre variedades Riemannianas com bordo
}

\author{
Elkin Dario Cardenas Diaz
}

TESE APRESENTADA

$\mathrm{AO}$

Instituto De Matemática e Estatística

DA

Universidade DE SÃo PaUlo

PARA

OBTENÇÃO DO TÍTULO

$\mathrm{DE}$

DOUTOR EM CIÊNCIAS

Programa: Doutorado em matemáticas

Orientador: Prof. Dr. Paolo Piccione

Durante o desenvolvimento deste trabalho o autor recebeu auxílio financeiro da CAPES

São Paulo, agosto de 2016 


\section{Fenômeno de bifurcação no problema de Yamabe sobre variedades Riemannianas com bordo}

Esta versão da tese contém as correções e alterações sugeridas pela Comissão Julgadora durante a defesa da versão original do trabalho, realizada em 16/08/2016. Uma cópia da versão original está disponível no

Instituto de Matemática e Estatística da Universidade de São Paulo.

Comissão Julgadora:

- Prof. Dr. Paolo Piccione (orientadora) - IME-USP

- Prof. Dr. Gaetano Siciliano - IME-USP

- Prof. Dr. Francesco Mercuri - UNICAMP

- Prof. Dr. Fernando Manfio - ICMC-US

- Prof. Dr. Marcelo Montenegro - UNICAMP 


\section{Agradecimentos}

Quando o caminho foi percorrido, uma olhada para trás permite perceber que são muitas as pessoas e acontecimentos que ajudaram chegar até onde você está. Na minha vida são muitas pessoas e acontecimentos que é impossível nomear cada uma de elas.

Mas, gostaria aproveitar o momento para agradecer algumas de elas e pedir desculpas para aqueles que não mencione.

Primeiramente agradeço a Deus, pois sem sua ajuda este objetivo não teria sido possível.

Minha gratidão para minha mulher Danela del Carmen Durango López, quem me motivou para continuar no curso de Doutorado e durante os últimos quatro anos assumiu o cuidado e educação de nossos filhos.

Não tem sido fácil para mi, mas sem duvida nenhuma tem sido mais árduo para ela. Meus filhos, Elias Daniel e Marcelo, também têm contribuído na minha consecução de esta meta e para eles minha gratitude. Agora espero não ter que ficar mais longe de vocês por tanto tempo.

Sou grato ao meu orientador de doutorado, professor Paolo Piccione, por ter me dado a oportunidade de trabalhar com ele. Sempre é bom ficar perto de pessoas com qualidades acadêmicas e pessoais como as de ele.

Agradeço a todas as pessoas maravilhosas que tenho conhecido no IME-USP, elas fizeram de meu tempo neste instituto mais agradável. Especialmente sou grato de minha colega Ana Claudia Moreira da Silva pela sua ajuda com algumas correções neste documento.

Finalmente agradeço meus pais, irmãos, colegas e todos aqueles que de uma ou outra forma me motivaram para chegar até aqui. 


\section{Resumo}

Cárdenas-Diaz, Elkin D. Fenômeno de bifurcação no problema de Yamabe sobre variedades Riemannianas com bordo. 2016. 120 f. Tese (Doutorado) - Instituto de Matemática e Estatística, Universidade de São Paulo, São Paulo, 2016.

No presente trabalho consideramos o produto de uma variedade Riemanniana compacta sem bordo de curvatura escalar zero e uma variedade Riemanniana compacta com bordo, curvatura escalar zero e curvatura media constante no bordo, e fazemos uso da teoria de bifurcação para provar a existência de um numero infinito de classes conforme com, pelo menos, duas métricas Riemannianas não homotéticas de curvatura escalar zero e curvatura média constante no bordo, sobre a variedade produto.

Palavras-chave: Variedades produto, problema de Yamabe, bifurcação, métricas Riemannianas, classes conforme. 


\section{Abstract}

Cárdenas-Díaz, Elkin D. Phenomenon of bifurcation in Yamabe problem on Riemannian manifolds with boundary. 2016. 120 f. Tese (Doutorado) - Instituto de Matematítica e Estatística, Universidade de São Paulo, São Paulo, 2016.

In this work, we consider the product of a compact Riemannian manifold without boundary, null scalar curvature and a compact Riemannian manifold with boundary, null scalar curvature and constant mean curvature on the boundary and we use the bifurcation theory to prove the existence of a infinite number of conformal classes with at least two non homothetic Riemannian metrics of null scalar curvature and constant mean curvature of the boundary on the product manifold.

Keywords: Product manifolds, Yamabe problem, bifurcation, Riemannian metrics, conformal class. 


\section{Sumário}

1 Introdução 1

2 Preliminares $\quad 5$

2.1 Geometria Riemanniana . . . . . . . . . . . . . . . . . . . 5

2.1 .1 Tensores sobre variedades $\ldots \ldots \ldots \ldots \ldots \ldots$

2.1 .2 Métricas e conexão Riemanniana . . . . . . . . . . . . . . . . . . . 7

2.1 .3 Curvatura . . . . . . . . . . . . . . . . . . . . . . 10

2.1.4 Integração sobre variedades Riemannianas . . . . . . . . . . . . . . . . 10

2.1 .5 Variedades com bordo . . . . . . . . . . . . . . . . . . 11

2.1 .6 Mudanças conforme . . . . . . . . . . . . . . . . . . 13

2.2 Análise Funcional . . . . . . . . . . . . . . . . . . . . . . . . . . . 16

2.2 .1 Teorema da função implícita . . . . . . . . . . . . . . . . . . 16

2.2 .2 Operadores Fredholm . . . . . . . . . . . . . . . . . 17

2.2 .3 Espaços de Sobolev sobre variedades . . . . . . . . . . . . . . . . . 18

2.2 .4 Variedades de Banach . . . . . . . . . . . . . . . . . . . . . 20

3 Teoremas de Bifurcação $\quad 23$

3.1 Aspectos gerais . . . . . . . . . . . . . . . . . . . . . . . . 23

3.1 .1 Metodo de Lyapunov-Schmidt . . . . . . . . . . . . . . . . . . 24

3.1 .2 Operadores Gradiente . . . . . . . . . . . . . . . . . 25

3.2 O teorema de Smoller-Wasserman . . . . . . . . . . . . . . . . . . . 26

3.3 Teorema de bifurcação sobre domínio variável . . . . . . . . . . . . . . . . . . 26

4 Fundamento variacional $\quad 29$

4.1 O operador de Jacobi . . . . . . . . . . . . . . . . . . . . . . . . 32

5 Critério de bifurcação no problema de Yamabe 35

5.1 Convergência num ramo de bifurcação . . . . . . . . . . . . . . 36

5.2 Existencia de instantes de bifurcação . . . . . . . . . . . . . . . . . . . . 39

6 Bifurcação das soluções do problema de Yamabe sobre variedades produto 41

$\begin{array}{ll}\text { A Um problema de autovalor } & 47\end{array}$

$\begin{array}{ll}\text { B Direchelt-Neumann } & 57\end{array}$ 
viii SUMÁRIO

Referências Bibliográficas 


\section{Capítulo 1}

\section{Introdução}

Hilbert em [Hilbert, 1915] provou que uma métrica $g$ sobre uma variedade compacta $M$ é Einstein se e só se é um ponto crítico do funcional normalizado de Hilbert-Einstein

$$
g \longrightarrow \mathcal{E}(g)=\frac{\int_{M} R_{g} v_{g}}{\operatorname{Vol}_{g}(M)^{\frac{m-2}{m}}},
$$

onde $R_{g}$ denota a curvatura escalar da métrica $g, v_{g}$ é a forma de volume induzida por $g, m$ é a dimensão da variedade $M$ e $\operatorname{Vol}_{g}(M)=\int_{M} v_{g}$. Agora, quando dito funcional é restrito a classe conforme $[g]$ da métrica $g$ obtemos que os pontos críticos são as métricas conforme à $g$ com curvatura escalar constante. A existência desses pontos críticos é o celebre problema de Yamabe.

Yamabe em [Yamabe , 1960] apresentou uma prova da seguinte afirmação:

Dada uma variedade Riemanniana $m$-dimensional $(M, g)$, compacta com $m \geq 3$, existe uma métrica conforme à $g$ com curvatura escalar constante.

Mas, infortunadamente, a demostração dada por Yamabe tinha um erro que foi descoberto por Trudinger [Trudinger , 1968]. Trudinger conseguiu refazer a prova de Yamabe com a imposição de algumas restriçõe sobre a variedade. Mais justamente, sobre a classe conforme $[g]$ o funcional $\mathcal{E}$ pode se escrever como

$$
\mathcal{E}(\tilde{g})=\mathcal{E}\left(\varphi^{\frac{4}{m-2}} g\right)=\mathcal{E}_{g}(\varphi)=\frac{E_{g}(\varphi)}{\|\varphi\|_{\frac{2 m}{m-2}}^{2}}
$$

onde

$$
E_{g}(\varphi)=\frac{4(m-1)}{m-2} \int_{M}\left(|\nabla \varphi|^{2}+\frac{m-2}{4(m-1)} R_{g} \varphi^{2}\right) v_{g} \quad \text { e }\|\varphi\|_{\frac{2 m}{m-2}}=\left(\int_{M} \varphi^{\frac{2 m}{m-2}} v_{g}\right)^{\frac{m-2}{2 m}} .
$$

A constante de Yamabe $Y(M,[g])$ relativa a classe conforme $[g]$ é definida como

$$
Y(M,[g])=\inf _{\varphi \in C_{+}^{\infty}(M)} \mathcal{E}_{g}(\varphi),
$$

onde $C_{+}^{\infty}(M)$ denota o subconjunto das funções positivas de $C^{\infty}(M)$. Trudinger provou em [Trudinger , 1968] que o argumento do Yamabe funciona quando $Y(M,[g]) \leq 0$.

A esfera $\mathbb{S}^{m}$ com a métrica padrão $g_{0}$ desempenha um papel importante no desenvolvimento do problema de Yamabe. Aubin em [Aubin , 1976] mostrou que se a constante de Yamabe $Y(M,[g])$ é menor do que a constante de Yamabe $Y\left(\mathbb{S}^{m},\left[g_{0}\right]\right)$ da esfera $\mathbb{S}^{m}$ relativa a classe conforme $\left[g_{0}\right]$ da métrica padrão $g_{0}$ então existe uma métrica conforme à $g$ com curvatura escalar constante. Aubin também demonstrou em [Aubin , 1976] que se $(M, g)$ não é localmente conformemente plana e $\operatorname{dim} M \geq 6$ então $Y(M,[g])<Y\left(\mathbb{S}^{m},\left[g_{0}\right]\right)$.

Finalmente, Schoen em [Schoen , 1984] completou a solução do problema de Yamabe. Exatamente, ele provou que se $(M, g)$ não é conformemente equivalente à esfera redonda e à dimensão de $M$ é 3,4 ou 5 ou se $M$ é localmente conformemente plana, então $Y(M,[g])<Y\left(\mathbb{S}^{m},\left[g_{0}\right]\right)$. 
O fato de que a constante de Yamabe $Y(M,[g])$ é um invariante da classe conforme $[g]$ garante que não existem duas métricas de curvatura escalar constante com sinais diferentes na classe conforme $[g]$. Além disso, não é difícil provar que se $Y(M,[g]) \leq 0$ então existe uma única métrica conforme à $g$ de curvatura escalar constante, exceto homotetias. Agora, quando $Y(M,[g])>0$ a situação é muito mais complicada. Por exemplo, Obata em [Obata, 1971] obteve resultados de unicidade para métricas de Einstein. Especificamente, ele provou que se a métrica $g$ é Einstein e $(M, g)$ não é isométrica à esfera redonda $\left(\mathbb{S}^{m}, g_{0}\right)$ então as métricas conforme $\tilde{g}$ à $g$ com curvatura escalar constante são proporcionais à $g$. Schoen em [Schoen , 1989] considerou o produto $\mathbb{S}^{1}(\tau) \times \mathbb{S}^{n-1}(1)$ da circunferência de comprimento $\tau, \mathbb{S}^{1}(\tau)$, com a esfera de raio $1, \mathbb{S}^{m-1}(1)$, e apresentou resultados de unicidade. Mais precisamente, ele mostrou que se $\tau \leq 2 \pi(m-2)^{-1 / 2}$ então a métrica produto é a única métrica, salvo homotetias, de curvatura escalar constante na sua classe conforme. Mas, em geral não temos unicidade quando a constante de Yamabe é positiva. Por exemplo, Pollack em [Pollack, 1993] provou que qualquer classe conforme com constante de Yamabe positiva pode se aproximar na $C^{0}$-topologia por uma classe conforme com um número arbitrariamente grande de métricas, não isométricas, de curvatura escalar constante. Schoen em [Schoen , 1989] também demostrou que se $\tau>2 \pi(m-2)^{-1 / 2}$, então existem múltiplas métricas, não homotéticas, de curvatura escalar constante na classe conforme da métrica produto sobre $\mathbb{S}^{1}(\tau) \times \mathbb{S}^{n-1}(1)$.

Recentemente diversas técnicas têm sido usadas por diferentes autores para garantir a multiplicidade de métricas de curvatura escalar constante na classe conforme de uma métrica dada. A título de exemplo, J. Petean em [Petean , 2010] usando técnicas de comparação de Sturm obteve resultados de multiplicidade para certo tipo de variadedes produto. Mais precisamente, Petean provou que dada uma variedade Riemanniana fechada $(M, g)$ de curvatura escalar constante $R>0$ o número de métricas, não isométricas, de volume unitário e curvatura escalar constante na classe conforme $\left[g_{0}+g\right]$ da métrica produto sobre $\mathbb{S}^{m} \times M$ cresce pelo menos linearmente com $\sqrt{R}$. De Lima, Piccione e Zedda em [Lima et al. , 2012] usaram a teoria da bifurcação para obter resultados de rigidez local e multiplicidade sobre produtos Riemanniananos. Eles consideraram o produto de duas variedades Riemannianas compactas sem bordo $\left(M_{i}, g^{(i)}\right), i=0,1$, de curvatura escalar constante e definiram a família de métricas $\left.g_{\lambda}, \lambda \in\right] 0,+\infty$ [, de curvatura escalar constante sobre a variedade produto $M=M_{0} \times M_{1}$, por $g_{\lambda}=g^{(0)} \oplus \lambda g^{(1)}$. Então eles provaram que existe um conjunto enumerável $\Lambda \subset] 0, \infty[$ que se acumulam se unicamente em 0 e $+\infty$ tal que:

- A família $\left(g_{\lambda}\right)$ é localmente rígida em todo ponto de $] 0,+\infty[\backslash \Lambda$, isto é, para cada $\bar{\lambda} \in] 0,+\infty[\backslash \Lambda$, qualquer métrica de curvatura escalar constante sobre $M$ o suficientemente $C^{2, \alpha}$-próxima à $g_{\bar{\lambda}}$ é homotética à algum elemento da família $\left(g_{\lambda}\right)$;

- Em cada $\lambda_{*} \in \Lambda$, exceto para um subconjunto finito, existe um ramo de bifurcação de métricas de curvatura escalar constante, emergindo de $g_{*}$, o qual é constituído por métricas que não pertencem à família $\left(g_{\lambda}\right)$.

Eles também obtêm resultados de rigidez local e bifurcação quando a curvatura escalar de $g^{(i)}, i=0,1$, não são, ambas positivas. Outros resultados de multiplicidade são obtidos em [Hebey e Vaugon , 1992], [Brendle, 2008] e [Khuri et al. , 2009].

Análogos ao problema de Yamabe para variedades Riemannianas compactas com bordo têm sido estudados, entre outros autores, por Cherrier [Cherrier , 1984], Escobar [Escobar , 1992a,b, 1996], Han e Li [Han e Li , 1999] e Marques [Marques , 2005, 2007].

Em particular, dada uma variedade Riemanniana compacta $(M, g)$ com bordo e dimensão $m \geq 3$, Escobar estudou em [Escobar , 1992b] o problema de encontrar uma métrica conforme $\tilde{g}=\varphi^{\frac{4}{m-2}} g$ à $g$ de curvatura escalar zero tal que o bordo $\partial M$ têm curvatura média $H_{\tilde{g}}$ constante. Este problema é equivalente à provar a existência de pontos críticos do funcional

$$
E(\varphi)=\frac{\int_{M}\left(g(\nabla \varphi, \nabla \varphi)+\frac{m-2}{4(m-1)} R_{g} \varphi^{2}\right) v_{g}+\frac{m-2}{2} \int_{\partial M} H_{g} \varphi^{2} \sigma_{g}}{\left(\int_{\partial M} \varphi^{\frac{2(m-1)}{m-2}} \sigma_{g}\right)^{\frac{m-2}{m-1}}},
$$


onde $v_{g}$ e $\sigma_{g}$ denotam as formas de volume sobre $M$ e $\partial M$ induzidas por $g$, respectivamente.

Escobar em [Escobar , 1992b] define o quociente de Sobolev $Q(M, \partial M)$ como sendo:

$$
Q(M, \partial M)=\inf \left\{E(\varphi) \mid \varphi \in C^{1}(\bar{M}), \varphi \neq 0 \text { sobre } \partial M\right\},
$$

e provou que $Q(M, \partial M)$ é um invariante conforme com

$$
Q(M, \partial M) \leq Q\left(B^{n}, \partial B^{n}\right)
$$

onde $B^{n}$ denota a bola unitária em $\mathbb{R}^{n}$ munida com a métrica Euclidiana. Além disso, ele mostrou que se o quociente de Sobolev $Q(M, \partial M)$ é finito e $Q(M, \partial M)<Q\left(B^{n}, \partial B^{n}\right)$, então existe uma métrica de curvatura escalar zero e curvatura média constante no bordo na classe conforme $[g]$ da métrica $g$. No mesmo artigo, Escobar garante a existência de uma classe suficientemente grande de variedades Riemannianas compactas com bordo que são conformalmente equivalentes à uma com curvatura escalar zero e curvatura média constante sobre o bordo. Escobar provou que toda variedade Riemanniana compacta $(M, g)$ com bordo e dimensão $m \geq 3$ que atende uma das seguintes condições

(i) $m>6$, e $M$ têm um ponto não umbílico sobre $\partial M$;

(ii) $m \geq 6$ com $M$ localmente conformalmente plana e $\partial M$ umbílica;

(iii) $m=4$ ou 5 , e $\partial M$ umbílica;

(iv) $m=3$;

é conformalmente equivalente à uma de curvatura escalar zero e curvatura média constante sobre o bordo.

Marques em [Marques, 2005, 2007] estabeleceu resultados de existência no caso de variedades com bordo umbílico e dimensão $m \geq 8$ e para variedades de dimensão $m=4$ ou 5 com bordo $\partial M$ não umbílico. Ele provou que dada uma variedade Riemanniana compacta $(M, g)$ com bordo $\partial M$ não vazio, se uma das seguintes condições é verdade:

(i) $m \geq 8, \bar{W}(x) \neq 0$ para algum $x \in \partial M$ e $\partial M$ é umbílico;

(ii) $m \geq 9, W(x) \neq 0$ para algum $x \in \partial M$ e $\partial M$ é umbílico;

(iii) $m=4$ ou 5 , e $\partial M$ é não umbílica;

então $Q(M, \partial M)<Q\left(B^{m}, \partial B^{m}\right)$. Além disso, se $Q(M, \partial M)$ é finito exite uma métrica $\tilde{g}$ conformalmente relacionada à $g$ com curvatura escalar zero e curvatura média constante sobre o bordo. Aqui $W$ e $\bar{W}$ denotam o tensor de Weyl sobre $M$ e $\partial M$, respectivamente.

Almaraz em [Almaraz, 2010] obteve um resultado de existência no caso de variedades com bordo umbílico e dimensão $m=6,7$ ou 8 . Mais exatamente, ele provou que se $m=6,7$ ou $8, \partial M$ é umbílico e $W(x) \neq 0$ para algum $x \in \partial M$ então $Q(M, \partial M)<Q\left(B^{m}, \partial B^{m}\right)$.

Da mesma forma que no caso de variedades fechadas, quando o quociente de Sobolev $Q(M, \partial M)$ é não positivo existe uma única métrica $\tilde{g}$, exceto homotetia, de curvatura escalar zero e curvatura media constante no bordo conformalmente relacionada com $g$. Mas no caso $Q(M, \partial M)>0$, em geral, não se tem unicidade. Alguns resultados de multiplicidade são obtidos em [Escobar , 2003], [García e Munoz, 2012] .

Nesta tese consideramos o produto de uma variedade Riemanniana compacta, sem bordo, e curvatura escalar zero com uma variedade Riemanniana compacta, com bordo, curvatura escalar zero e curvatura média constante no bordo e estabelecemos resultados de multiplicidades de métricas de curvatura escalar zero e curvatura média constante no bordo num número infinito de classes conforme. Para provar nosso resultado principal usamos a teoria da bifurcação seguindo a estratégia desenvolvida por Lima, Piccione e Zedda em [Lima et al., 2012]. A seguir, descrevemos nosso resultado principal mais precisamente. Dadas as variedades Riemannianas compactas $\left(M_{1}, g^{(1)}\right)$, 
sem bordo e curvatura escalar zero e $\left(M_{2}, g^{(2)}\right)$, com bordo, curvatura escalar zero e curvatura média constante no bordo. Suponha que $M_{i}, i=1,2$, é orientada e consideramos a família de métricas $\left(g_{t}\right)_{t \in] 0,+\infty}$, de curvatura escalar zero e curvatura média constante no bordo, sobre a variedade produto $M=M_{1} \times M_{2}$, definidas por $g_{t}=g^{(1)} \oplus t g^{(2)}$. Nosso resultado principal diz que existe uma sequência convergindo a zero consistindo de instantes de bifurcação para a família $\left(g_{t}\right)_{t \in] 0,+\infty[} \cdot$ 


\section{Capítulo 2}

\section{Preliminares}

O objetivo deste capítulo é apresentar, de forma breve, alguns conceitos da geometria Riemanniana e da análise funcional que estão misturados no desenvolvimento deste trabalho.

Iniciamos o capítulo apontando os conceitos da geometria diferencial e Riemanniana de nosso interesse. Na subseção 2.1.6 apontamos as fórmulas de transformação da conexão Riemanniana e do tensor de curvatura de Ricci associados a uma variedade Riemanniana quando consideramos uma mudança conforme da métrica. A partir dessas fórmulas de transformação obtemos fórmulas de mudança para a curvatura escalar e a curvatura média do bordo.

Na seção 2.2 expomos os fundamentos da análise funcional que precisaremos nos capítulos seguintes. Particularmente, falamos sobre os operadores de Fredholm, os espaços de Sobolev sobre variedades Riemannianas e finalizamos o capítulo falando sobre as variedades de Banach.

As principais referências deste capítulo são [Druet et al. , 2004], [Petersen, 1998], [Carmo , 2005], [Lee , 1997], [Besse , 2008], [Brezis , 2011], [Abramovich e Aliprantis , 2002], [Ambrosetti e Prodi , 1993], [Hebey , 2000], [Lions e Magenes , 1972], [Taylor , 2011a], [Leoni , 2009], [Kesavan , 2003].

\subsection{Geometria Riemanniana}

Começamos a seção com alguns conceitos da geometria diferencial. Um espaço topológico Haussdorff, que satisfaz o segundo axioma de enumerabilidade $M$ é dito uma variedade topológica de dimensão ${ }^{1} m$ se cada ponto de $M$ possui uma vizinhançã aberta que é homeomorfo à um aberto do espaço euclidiano $\mathbb{R}^{m}$. Uma carta coordenada ou simplesmente carta de $M$ é um par $(\Omega, \varphi)$ onde $\Omega$ denota um subconjunto aberto de $M$ e $\varphi$ num homeomorfismo de $\Omega$ sobre um subconjunto aberto de $\mathbb{R}^{m}$. O aberto $\Omega$ é chamado uma vizinhança coordenada (de $M$ ), e $\varphi$ uma função coordenada ou sistema coordenado sobre $\Omega$. Para $p \in \Omega$ as coordenadas de $\varphi(p)$ em $\mathbb{R}^{m}$ são chamadas coordenadas de $p$ na carta $(\Omega, \varphi)$. Um atlas de $M$ é uma coleção de cartas $\mathcal{U}=\left\{\left(\Omega_{i}, \varphi_{i}\right)\right\}, i \in I$, tal que $M=\cup_{i \in I} \Omega_{i}$. Dadas duas cartas $\left(\Omega_{i}, \varphi_{i}\right)$ e $\left(\Omega_{j}, \varphi_{j}\right)$ de um atlas $\left\{\left(\Omega_{i}, \varphi_{i}\right)\right\}$, com $\Omega_{i} \cap \Omega_{j} \neq \emptyset$ as funções

$$
\varphi_{i j}=\varphi_{i} \circ \varphi_{j}^{-1}: \varphi_{j}\left(\Omega_{j} \cap \Omega_{i}\right) \longrightarrow \varphi_{i}\left(\Omega_{j} \cap \Omega_{i}\right), \quad \varphi_{j i}=\varphi_{j} \circ \varphi_{i}^{-1}: \varphi_{i}\left(\Omega_{j} \cap \Omega_{i}\right) \longrightarrow \varphi_{j}\left(\Omega_{j} \cap \Omega_{i}\right)
$$

são chamadas funções de transição entre as ditas cartas. Se as funções de transição num atlas são de classe $C^{k}$ o atlas é dito de classe $C^{k}$. O atlas é dito $C^{k}$-completo se não está contido (estritamente) num atlas de classe $C^{k}$ maior. Não é difícil provar que todo atlas de classe $C^{k}$ está contido num único atlas $C^{k}$-completo. Apontamos agora a definição de variedade de classe $C^{k}$. Uma variedade $m$-dimensional de classe $C^{k}$ é uma variedade topológica de dimensão $m$ munida com um atlas $C^{k}$ completo. No decorrer desta tese vamos supor $k=+\infty, M$ conexa e nos referiremos a $M$ como uma variedade $m$-dimensional suave.

\footnotetext{
${ }^{1}$ Para garantir que a dimensão de uma variedade topológica está bem definida, precisamos mostrar que um aberto de $\mathbb{R}^{m}$ não pode ser homeomorfo à um aberto de $\mathbb{R}^{n}$, se $m \neq n$. Este fato é consequência de um teorema mais geral, mas não fácil de provar, conhecido como teorema de invariância do domínio e provado pelo J. Brouwer em 1912.
} 
Dada uma função $f: M \longrightarrow N$ entre duas variedades suaves, $M$ e $N$, dizemos que $f$ é diferenciável (ou de classe $C^{r}$ ) se para quaisquer duas cartas $(\Omega, \varphi),(\Lambda, \psi)$ de $M$ e $N$, respectivamente, tal que $f(\Omega) \subset \Lambda$, a função

$$
\psi \circ f \circ \varphi^{-1}: \varphi(\Omega) \longrightarrow \psi(\Lambda),
$$

é diferenciável (ou de classe $C^{r}$ ). Uma função suave entre variedades é simplesmente uma função de classe $C^{\infty}$. Denotaremos por $C^{\infty}(M)$ o espaço das funções suaves de $M$ em $\mathbb{R}$. Uma função $f: M \longrightarrow N$ é um difeomorfismo se ela é diferenciável, biunívoca, sobrejetiva e sua inversa $f^{-1}$ é diferenciável. Duas variedades suaves $M$ e $N$ são difeomorfas se existe um difeomorfismo entre elas.

Dadas $f, g \in C^{\infty}(M)$ e $p \in M$, dizemos que $f$ é $p$-equivalente à $g$ se existe uma vizinhança aberta $U_{p}$ de $p$ tal que $f=g$ sobre $U_{p}$. É fácil ver que a $p$-equivalência define uma relação de equivalência $\sim$ sobre $C^{\infty}(M)$. O germe de $f$ em $p$ é a classe de equivalência de todas as funções $p$-equivalentes com $f$. Denotamos por $C_{p}^{\infty}(M)=C^{\infty}(M) / \sim$. Um vetor tangente no ponto $p \in M$ é uma função $\mathbb{R}$-linear ${ }^{2} X_{p}: C_{p}^{\infty}(M) \longrightarrow \mathbb{R}$ tal que

$$
X_{p}(f g)=X_{p}(f) g(p)+f(p) X_{p}(g), \quad f, g \in C_{p}^{\infty}(M) .
$$

O espaço vetorial de todos os vetores tangentes de $M$ em $p$, denotado por $T_{p} M$, é chamado o espaço tangente de $M$ em $p$. Dada uma carta coordenada $(\Omega, \varphi)$, com $p \in \Omega$, definimos $\left.\partial_{i}\right|_{p} \in T_{p} M$ como sendo

$$
\left.\partial_{i}\right|_{p}(f)=D_{i}\left(f \circ \varphi^{-1}\right)_{\varphi(p)} .
$$

Aqui $D_{i}$ denota a $i$-ésima derivada parcial. Os vetores $\left.\partial_{i}\right|_{p}, i=1, \ldots, m$, formam uma base para o espaço tangente $T_{p} M$. Dada duas variedades suaves, $M$ e $N$, uma função diferenciável $f: M \longrightarrow N$ e um ponto $p \in M$, a diferencial de $f$ em $p$ é a função $\mathbb{R}$-linear $d f_{p}$ do espaço tangente $T_{p} M$ no espaço tangente $T_{f(p)} N$ dada por

$$
d f_{p}\left(X_{p}\right)(g)=X_{p}(g \circ f), \quad X_{p} \in T_{p} M, g \in C_{f(p)}^{\infty}(N) .
$$

O fibrado tangente $T M$ se define como a união disjunta dos $T_{p} M, p \in M$. Se $M$ é $m$-dimensional, não é difícil provar que $T M$ tem estrutura de variedade $2 m$-dimensional suave. Um campo vetorial sobre $M$ é uma função $X: M \longrightarrow T M$ tal que $X(p) \in T_{p} M$, para todo $p \in M$. Dada uma carta $(\Omega, \varphi)$ de $M$, para cada $i=1, \ldots, m$, seja $\partial_{i}: \Omega \subset M \longrightarrow T M$ definido por $\partial_{i}(p)=\left.\partial_{i}\right|_{p}, p \in \Omega$, é um campo vetorial sobre $\Omega$. O conjunto $\left\{\partial_{i}\right\}_{i=1}^{m}$ é chamado um referencial para $T M$ sobre $\Omega$. O espaço de todos os campos vetoriais suaves sobre $M$ será denotado por $\mathfrak{X}(M)$. Uma função diferenciável $f: M \longrightarrow N$ é dita uma imersão em $p \in M$ se a sua diferencial $d f_{p}: T_{p} M \longrightarrow T_{f(p)} N$ é injetora, e uma submersão em $p$ se $d f_{p}$ é sobrejetora. Diremos que $f$ é uma imersão se é uma imersão em todo ponto $p \in M$ e uma submersão se é uma submersão em todo ponto $p \in M$.

\subsubsection{Tensores sobre variedades}

Dado que alguns dos objetos importantes sobre uma variedade suave têm um carácter tensorial, diremos alguns palavras acerca dos tensores sobre uma variedade suave. O fibrado cotangente $T M^{*}$ se define como a união disjunta $\operatorname{dos} T_{p} M^{*}, p \in M$. Analogamente ao fibrado tangente, o fibrado cotangente tem estrutura de variedade $2 m$-dimensional suave, quando $M$ é uma variedade $m$-dimensional suave. Uma 1-forma sobre $M$ é uma função $w: M \longrightarrow T M^{*}$ tal que $w(p) \in T_{p} M^{*}$, para todo $p \in M$. Por exemplo, seja $f: M \longrightarrow \mathbb{R}$ uma função diferenciável, então $d f: M \longrightarrow T M^{*}$ é a 1-forma definida por $d f(p)=d f_{p}$. Dada uma carta $(\Omega, \varphi)$, seja $\left\{\left.\sigma^{i}\right|_{p}\right\}_{i=1}^{m}$ a base dual de $\left\{\left.\partial_{i}\right|_{p}\right\}_{i=1}^{m}, p \in \Omega$. Então $\sigma^{i}: \Omega \longrightarrow T M^{*}$ definida por $\sigma^{i}(p)=\left.\sigma^{i}\right|_{p}, i=1, \ldots, m$, define uma 1 -forma sobre $\Omega$. Chamaremos à $\left\{\sigma^{i}\right\}_{i=1}^{m}$ o referencial dual do referencial $\left\{\partial_{i}\right\}_{i=1}^{m}$ para $T M$ sobre $\Omega$. Denotaremos por $\mathfrak{X}(M)^{*}$ o espaço das 1 -forma suaves sobre $M$. Dada a variedade $m$-dimensional

\footnotetext{
${ }^{2} \mathbb{R}$-linear quer dizer que $X_{p}(\alpha f+\beta g)=\alpha X_{p}(f)+\beta X_{p}(g)$ para todo $\alpha, \beta \in \mathbb{R}$ e $f, g \in C_{p}^{\infty}(M)$.
} 
suave $M$ e $p \in M$, um (s,l)-tensor em $p$ é uma função multilinear

$$
F_{p}: \underbrace{T_{p} M \times \ldots \times T_{p} M}_{s \text { vezes }} \times \underbrace{T_{p} M^{*} \times \ldots \times T_{p} M^{*}}_{l \text { vezes }} \longrightarrow \mathbb{R} .
$$

O espaço dos $(s, l)$ - tensores em $p$ será denotado por $T_{l}^{s}\left(T_{p} M\right)$. Definimos o fibrado dos $(s, l)$-tensores sobre $M, T_{l}^{s} M$ como sendo a união disjunta dos $T_{l}^{s}\left(T_{p} M\right)$, com $p$ percorrendo $M$. $T_{l}^{s} M$ também tem estrutura de variedade $m\left(1+m^{s+l-1}\right)$-dimensional suave. Um $(s, l)$-campo tensorial sobre $M$ é uma função $F: M \longrightarrow T_{l}^{s} M$ tal que $F(p) \in T_{l}^{s}\left(T_{p} M\right)$, para todo $p \in M$. Denotaremos por $\Gamma_{l}^{s}(M)$ o espaço dos $(s, l)$-campos tensoriais suaves sobre $M$.

Dado um espaço vetorial finito dimensional $V$, sabemos pela álgebra linear que existe um isomorfismo natural (independente de base) entre $T_{1}^{1}(V)$, o espaço dos $(1,1)$-tensores sobre $V$, e $\operatorname{End}(V)$, o espaço dos endomorfismos de $V$. Este fato, nos permite definir o traço de $F \in T_{1}^{1}(V)$ como o traço de $F$ quando é considerado como um endomorfismo de $V$. Mais geral, dado $F \in T_{l+1}^{s+1}(V)$ definimos $\operatorname{tr}(F) \in T_{l}^{s}(V)$ como

$$
\operatorname{tr}(F)\left(X_{1}, \ldots, X_{s}, w^{1}, \ldots, w^{l}\right)=\operatorname{traço~} F\left(X_{1}, \ldots, X_{s}, \cdot, w^{1}, \ldots, w^{l}, \cdot\right) \in \operatorname{End}(V),
$$

onde $X_{i} \in V, i=1, \ldots, s$ e $w^{j} \in V^{*}, j=1, \ldots, l$. Dada uma variedade $m$-dimensional suave $M$ e $F \in \Gamma_{l+1}^{s+1}(M)$ definimos $\operatorname{tr}(F) \in \Gamma_{l}^{s}(M) \operatorname{como} \operatorname{tr}(F)(p)=\operatorname{tr}(F(p))$. Dadas duas variedades suaves $M$ e $N$, uma função suave $f: M \longrightarrow N$, e um $(s, 0)$-campo tensorial $T$ sobre $N$, definimos o pullback $f^{*} T$ de $T$ por $f$, como o $(s, 0)$-campo tensorial sobre $M$ definido para $p \in M$ e $X_{1}, \ldots, X_{s} \in T_{p} M$ por

$$
f^{*} T(p)\left(X_{1}, \ldots, X_{s}\right)=T(f(p))\left(d f(p)\left(X_{1}\right), \ldots, d f(p)\left(X_{s}\right)\right) .
$$

O seguinte lema fornece uma caracterização, muito útil, dos campos tensoriais.

Lema 2.1. (Caracterição dos campos tensoriais) Uma função

$$
\tau: \underbrace{\mathfrak{X}(M) \times \ldots \times \mathfrak{X}(M)}_{\text {s vezes }} \times \underbrace{\mathfrak{X}(M)^{*} \times \ldots \times \mathfrak{X}(M)^{*}}_{\text {l vezes }} \longrightarrow C^{\infty}(M),
$$

é induzida por um $(s, l)$-campo tensorial se e só se é multilinear sobre $C^{\infty}(M)$. Da mesma forma, a função

$$
\tau: \underbrace{\mathfrak{X}(M) \times \ldots \times \mathfrak{X}(M)}_{\text {s vezes }} \times \underbrace{\mathfrak{X}(M)^{*} \times \ldots \times \mathfrak{X}(M)^{*}}_{\text {l vezes }} \longrightarrow \mathfrak{X}(M),
$$

é induzida por um $(s, l+1)$-campo tensorial se e só se é multilinear sobre $C^{\infty}(M)$.

\subsubsection{Métricas e conexão Riemanniana}

Agora abordamos a geometria Riemanniana. Dada uma variedade $m$-dimensional suave $M$, uma métrica Riemanniana $g$ de classe $C^{k}$ sobre $M$ é um $(2,0)$-campo tensorial de classe $C^{k}$ sobre $M$ tal que para todo $p \in M, g(p)$ é um produto escalar sobre $T_{p} M$. Uma variedade Riemanniana $m$-dimensional é um par $(M, g)$ onde $M$ é uma variedade $m$-dimensional suave e $g$ uma métrica Riemanniana sobre $M$. A seguinte proposição garante a existência de métricas Riemannianas.

Proposição 2.1. Toda variedade suave $M$ admite uma métrica Riemanniana suave.

Duas variedades Riemannianas suaves $(M, g)$ e $(N, h)$ são ditas isométricas se existe um difeomorfismo $\varphi: M \longrightarrow N$ tal que $g=\varphi^{*} h$.

Seja $(M, g)$ uma variedade Riemanniana $m$-dimensional suave. Fazendo uso da estrutura Riemanniana podemos mudar o tipo de qualquer campo tensorial sobre $M$. Com efeito, a estrutura Riemanniana permite definir um isomorfismo natural entre $T M$ e $T M^{*}$ por

$$
T M \ni X \longrightarrow X^{b} \in T M^{*}
$$


onde

$$
X^{b}(p)(Y)=g(p)(X(p), Y), \quad p \in M, Y \in T_{p} M .
$$

Seja $w \in \mathfrak{X}(M)^{*}$, denotaremo por $w^{\#}$ o campo vectorial definido pela propriedade

$$
w(p)(X)=g(p)\left(w^{\#}(p), X\right), \quad p \in M, X \in T_{p} M .
$$

Usando este isomorfismo podemos câmbiar $T M$ por $T M^{*}$, ou vice-versa, para mudar o tipo de qualquer tensor. Mais especificamente, Dada uma carta $(\Omega, \varphi)$ qualquer $(s, l)$-campo tensorial $F$ sobre $M$, pode se expressar (em $\Omega$ ) como

$$
F=F_{j_{1} \ldots j_{s}}^{i_{1} \ldots i_{l}} \sigma^{j_{i}} \otimes \ldots \otimes \sigma^{j_{s}} \otimes \partial_{i_{l}} \otimes \ldots \otimes \partial_{i_{1}}
$$

Na equação (2.1) estamos usado a notação conhecida como soma de Einstein, a qual estabelece que: numa expressão um índice que aparece dua vezes, sobrescrito e subscrito, é somado sobre todos os possíveis valores do mesmo. Agora a 1 -forma dual $\partial_{i}^{b}$ de $\partial_{i}$ pode se escrever (em $\Omega$ ) como

$$
\partial_{i}^{b}(V)=g\left(\partial_{i}, V^{j} \partial_{j}\right)=g\left(\partial_{i}, \partial_{j}\right) \sigma^{j}(V)=g_{i j} \sigma^{j}(V),
$$

onde $V=V^{j} \partial_{j}$ e $g_{i j}=g\left(\partial_{i}, \partial_{j}\right)$. Portanto $\partial_{i}^{b}=g_{i j} \sigma^{j}$. Também temos que o campo de vetores dual, de vetores, $\sigma^{i \#}$ da 1 -forma $\sigma^{i}$ é dado por

$$
\delta_{j}^{i}=\sigma^{i}\left(\partial_{j}\right)=g\left(\sigma^{i \#}, \partial_{j}\right),
$$

onde $\delta_{j}^{i}$ denota a delta de Kronecker. Escrevendo $\sigma^{i \#}=w^{r} \partial_{r}$ obtemos

$$
\delta_{j}^{i}=w^{r} g_{r j} .
$$

Denotando por $g^{i j}$ a $(i, j)$-componente da inversa da matriz $\left(g_{i j}\right)$ podemos escrever

$$
\sigma^{i \#}=g^{i r} \partial_{r}
$$

Logo $F$ pode se considerado como o $(s+1, l-1)$-campo tensorial

$F=F_{i_{1} \ldots i_{s}}^{j_{1} \ldots j_{l}} g_{j_{l} i_{s+1}} \sigma^{i_{1}} \otimes \ldots \otimes \sigma^{i_{s+1}} \otimes \partial_{j_{l-1}} \otimes \partial_{j_{l}} \otimes \ldots \otimes \partial_{j_{1}}=\hat{F}_{i_{1} \ldots i_{s+1}}^{j_{1} \ldots j_{l-1}} \sigma^{i_{1}} \otimes \ldots \otimes \sigma^{i_{s+1}} \otimes \partial_{j_{l-1}} \otimes \partial_{j_{l}} \otimes \ldots \otimes \partial_{j_{1}}$,

onde

$$
\hat{F}_{i_{1} \ldots i_{s+1}}^{j_{1} \ldots j_{l-1}}=F_{i_{1} \ldots i_{s}}^{j_{1} \ldots j_{l}} g_{j_{l} i_{s+1}} .
$$

De maneira análoga, podemos considerar $F$ o $(s-1, l+1)$-campo tensorial dado por

$$
F=F_{i_{1} \ldots i_{s-1}}^{j_{1} \ldots j_{l+1}} \sigma^{i_{1}} \otimes \ldots \otimes \sigma^{i_{s-1}} \otimes \partial_{j_{l+1}} \otimes \partial_{j_{l}} \otimes \ldots \otimes \partial_{j_{1}},
$$

onde $F_{i_{1} \ldots i_{s-1}}^{j_{1} \ldots j_{l+1}}=F_{i_{1} \ldots i_{s}}^{j_{1} \ldots j_{l}} g^{i_{s} j_{l+1}}$. Por indução conseguimos escrever $F$ como um $(s+r, l-r)$-campo tensorial, para qualquer $r \in \mathbb{Z}$ tal que $s+r$ e $l-r$ são ambos não negativos. Como uma aplicação deste fato, dada uma variedade Riemanniana $(M, g)$ e um $(2,0)$-campo tensorial simétrico ${ }^{3} F$ sobre $M$, usando a estrutura Riemanniana podemos conceber $F$ como um $(1,1)$-campo tensorial $F^{\#}$ sobre $M$, e portanto definimos o traço de $F$ com respeito à $g$, denotado por $\operatorname{tr}_{g} F$, como o traço de $F^{\#}$.

Outro fato importante é que também podemos usar a estrutura Riemanniana para induzir um produto interno $\langle\cdot, \cdot\rangle_{g}$ sobre cada fibra $T_{l}^{s}\left(T_{p} M\right)$ do fibrado $T_{l}^{s}(M)$, o fibrado dos $(s, l)$-tensores sobre $M$, o qual varia suavemente no sentido que para qualquer dois campos tensoriais $F, G \in \Gamma_{l}^{s}(M)$

\footnotetext{
${ }^{3} \mathrm{Um}(2,0)$-campo tensorial $F$ sobre $M$ é dito simétrico se para todo $p \in M$,
}

$$
F(p)(X, Y)=F(p)(Y, X), X, Y \in T_{p} M .
$$


o produto interno $\langle F, G\rangle_{g}$ é uma função suave sobre $M$. Com efeito, numa carta $(\Omega, \varphi)$ de $M$ temos que $\langle,\rangle_{g}$ é dado por

$$
\langle F, G\rangle_{g}=g^{i_{1} r_{1}} \cdots g^{i_{s} r_{s}} g_{j_{1} t_{1}} \cdots g_{j_{l} t_{l}} F_{i_{1} \cdots i_{s}}^{j_{1} \cdots j_{l}} G_{r_{1} \cdots r_{s}}^{t_{1} \cdots t_{l}} .
$$

Não é difícil provar que para 1-formas $\omega$ e $\theta$ temos

$$
\langle\omega, \theta\rangle_{g}=g\left(\omega^{\#}, \theta^{\#}\right) .
$$

Quando não houver risco de confusão escreveremos simplesmente $\langle\cdot, \cdot\rangle$ no lugar de $\langle\cdot, \cdot\rangle_{g}$.

Definimos agora a noção de conexão Riemanniana. Seja $(M, g)$ uma variedade Riemanniana. Uma conexão Riemanniana sobre $(M, g)$ é uma aplicação

$$
\nabla: \mathfrak{X}(M) \times \mathfrak{X}(M) \longrightarrow \mathfrak{X}(M),
$$

que se indica por $(X, Y) \longrightarrow \nabla_{X} Y$ e que satisfaz as seguintes propriedades:

a) $\nabla_{f X+g Y} Z=f \nabla_{X} Z+g \nabla_{Y} Z$,

b) $\nabla_{X}(Y+Z)=\nabla_{X} Y+\nabla_{X} Z$,

c) $\nabla_{X} f Y=f \nabla_{X} Y+X(f) Y$,

d) $X g(Y, Z)=g\left(\nabla_{X} Y, Z\right)+g\left(Y, \nabla_{X} Z\right)$,

e) $\nabla_{X} Y-\nabla_{Y} Z=[X, Y]$

onde $X, Y, Z \in \mathfrak{X}(M)$ e $f, g \in C^{\infty}(M)$. Uma aplicação que satisfaz (a)-(c) é chamada uma conexão linear. O seguinte teorema é um dos resultados fundamentais da geometria Riemanniana

Teorema 2.1. Sobre uma variedade Riemanniana $(M, g)$ existe uma e só uma conexão Riemanniana.

Dada uma variedade Riemanniana $(M, g)$ denotaremos por $\nabla^{g}$ sua conexã Riemanniana. Algumas vezes, quando não houver risco de confusão, usaremos $\nabla$ no lugar de $\nabla^{g}$.

Dada uma carta $(\Omega, \varphi)$ podemos expressar

$$
\nabla_{\partial_{i}} \partial_{j}=\Gamma_{i j}^{s} \partial_{s}
$$

onde

$$
\Gamma_{i j}^{s}=\frac{1}{2} g^{s l}\left(\partial_{i} g_{l j}+\partial_{j} g_{i l}-\partial_{l} g_{i j}\right) .
$$

As funções $\Gamma_{i j}^{s}: \Omega \longrightarrow \mathbb{R}$ são chamados os símbolos de Christoffel da conexão $\nabla$ na carta $(\Omega, \varphi)$.

A conexão $\nabla$ pode se estender sobre campos tensoriais como segue: Dado um $(s, l)$-campo tensorial de classe $C^{k}$ sobre $M, F$ e uma carta $(\Omega, \varphi)$, denotamos por $\nabla_{i} F$ o $(s, l)$-campo tensorial de classe $C^{k-1}$ sobre M, cujas componentes $\left(\nabla_{i} F\right)_{i_{1} \cdots i_{s}}^{j_{1} \cdots j_{l}}(\operatorname{em}(\Omega, \varphi))$ são dadas por

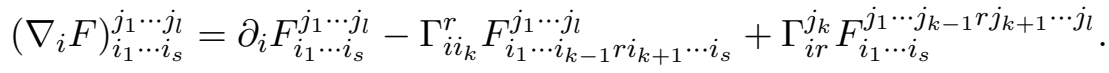

Finalmente, dado o $(s, l)$-campo tensorial $F$, a derivada covariante de $F$, denotada por $\nabla F$, é o $(s+1, l)$-campo tensorial cujas componentes na carta $(\Omega, \varphi)$ são dadas por

$$
(\nabla F)_{i_{1} \cdots i_{s+1}}^{j_{1} \cdots j_{l}}=\left(\nabla_{i_{1}} F\right)_{i_{2} \cdots i_{s+1}}^{j_{1} \cdots j_{l}}
$$

Por indução, podemos definir $\nabla^{2}, \nabla^{3}, \ldots$ Chamaremos à $\nabla^{k}, k \in \mathbb{N}, k$-ésima derivada covariante. Em particular, dada uma função suave $f: M \longrightarrow \mathbb{R}$, temos que $\nabla f$ é a 1 -forma $d f$. Com efeito,

$$
(\nabla f)(X)=\nabla_{X} f=X(f)=d f(X), \quad X \in \mathfrak{X}(M) .
$$

O Hessiano de $f$ é sua segunda derivada covariante, isto é, o $(2,0)$-campo tensorial $\nabla^{2} f$. Muitas vezes usamos $\operatorname{Hess}_{f}$ para denotar $\nabla^{2} f$. 


\subsubsection{Curvatura}

Passamos a definir agora o conceito de curvatura sobre uma variedade Riemanniana $(M, g)$. O tensor de curvatura é o $(3,1)$-campo tensorial dado por

$$
R(X, Y) Z=\nabla_{X} \nabla_{Y} Z-\nabla_{Y} \nabla_{X} Z-\nabla_{[X ; Y]} Z, \quad X, Y, Z \in \mathfrak{X}(M) .
$$

A curvatura Riemanniana ou curvatura de Riemann da métrica $g$ é o $(4,0)$-tensor definido por

$$
\operatorname{Riem}_{g}(X, Y, Z, W)=g(R(X, Y) Z, W)=g\left(\nabla_{X} \nabla_{Y} Z-\nabla_{Y} \nabla_{X} Z-\nabla_{[X ; Y]} Z, W\right),
$$

$X, Y, Z, W \in \mathfrak{X}(M)$. O tensor de Ricci é o $(2,0)$-tensor simétrico definido por

$$
\operatorname{Ric}_{g}(X, Y)=\operatorname{traço}(V \longrightarrow R(X, V) Y) .
$$

A curvatura escalar $R_{g}$ da métrica $g$ é definida como o traço com respeito à $g$ do tensor de Ricci. Isto é,

$$
R_{g}=t r_{g} \operatorname{Ric}_{g}
$$

Numa carta coordenada $(\Omega, \varphi)$ temos

$$
\operatorname{Riem}_{g}=R_{i j k l}\left(d x^{i} \wedge d x^{j}\right) \otimes\left(d x^{k} \wedge d x^{l}\right) \quad \text { e } \quad \operatorname{Ric}_{g}=R_{i j} d x^{i} \otimes d x^{j}
$$

onde

Além disso,

$$
R_{i j k l}=\operatorname{Riem}_{g}\left(\partial_{i}, \partial_{j}, \partial_{k}, \partial_{l}\right) \quad \text { e } \quad R_{i j}=g^{k s} R_{i k j s} .
$$

$$
R_{g}=g^{i j} R_{i j}
$$

\subsubsection{Integração sobre variedades Riemannianas}

Agora abordaremos a integração sobre uma variedade Riemanniana. Para isto, faremos algumas definições prévias. Um atlas de uma variedade suave $M$ é dito orientado se para quaisquer duas cartas $\left(\Omega_{i}, \varphi_{i},\right) i=1,2$, com $\Omega_{1} \cap \Omega_{2} \neq \emptyset$, a função de transição $\varphi_{2} \circ \varphi_{1}^{-1}$ tem determinante jacobiano positivo em todo ponto de $\varphi_{1}\left(\Omega_{1} \cap \Omega_{2}\right)$. A variedade suave $M$ é orientável se possui um atlas orientado. Dois atlas orientados $\left\{\left(\Omega_{i}, \varphi_{i}\right)\right\}$ e $\left\{\left(\Lambda_{j}, \psi_{j}\right)\right\}$ de $M$ são equivalentes se para quaisquer duas cartas $\left(\Omega_{i}, \varphi_{i}\right)$ e $\left(\Lambda_{j}, \psi_{j}\right)$ com $\Omega_{i} \cap \Lambda_{j} \neq \emptyset$ a função de transição $\psi_{j} \circ \varphi_{i}^{-1}$ tem determinante jacobiano positivo. Não é difícil provar que isto define uma relação de equivalência sobre o conjunto dos atlas orientados sobre uma variedade $M$. Existem duas dessas classes de equivalência. Escolhendo uma dessa duas classes de equivalência definimos uma orientação sobre $M$, neste caso $M$ é chamada orientada. Diremos que a variedade Riemanniana $(M, g)$ está orientada se $M$ é orientada.

Seja $(M, g)$ uma variedade Riemanniana $m$-dimensional orientada, e $\mathcal{A}=\left\{\left(\Omega_{i}, \varphi_{i}\right)\right\}_{i \in I}$ um atlas de $M$ compatível com a orientação. A $m$-forma de volume $v_{g}$ induzida por $g$ é definida na $\operatorname{carta}(\Omega, \varphi) \in \mathcal{A}$ por

$$
v_{g}=\sqrt{\operatorname{det}\left(g_{i j}\right)} d x^{1} \wedge d x^{2} \wedge \cdots \wedge d x^{m},
$$

onde

$$
\left(d x^{1} \wedge d x^{2} \wedge \cdots \wedge d x^{m}\right)\left(X_{1}, \ldots, X_{m}\right)=\operatorname{det}\left(a_{i j}\right), \quad a_{i j}=d x^{i}\left(X_{j}\right), X_{j} \in \mathfrak{X}(M) .
$$

$v_{g}$ é independente da carta e portanto globalmente definida. Com efeito, dada outra carta qualquer $(\Lambda, \psi) \in \mathcal{A}$, tal que $\Omega \cap \Lambda \neq \emptyset$, denotaremos por $\left\{\tilde{\partial}_{i}\right\}_{i=1}^{m}$ o referencial de $T M$ sobre $\Lambda$ e por $\left\{d y^{i}\right\}_{i=1}^{m}$ seu referencial dual. Seja $\tilde{v}_{g}=\sqrt{\operatorname{det}\left(\tilde{g}_{i j}\right)} d y^{1} \wedge d y^{2} \wedge \cdots \wedge d y^{m}$, onde $\tilde{g}_{i j}$ denota as componentes da métrica $g$ na carta $(\Lambda, \psi)$. Observe se que

$$
\tilde{\partial}_{j}=\tilde{\partial}_{j}\left(\pi^{s} \circ \varphi\right) \partial_{s} \quad \text { e } \quad d y^{j}=\partial_{s}\left(\pi^{j} \circ \psi\right) d x^{s},
$$


onde $\pi^{j}, j=1, \ldots, m$, denota a $j$-ésima projeção sobre $\mathbb{R}^{m}$. Sejam $A=\left(A_{i}^{j}\right), B=\left(B_{i}^{j}\right)$ com

$$
A_{i}^{j}=\tilde{\partial}_{i}\left(\pi^{j} \circ \varphi\right) \quad \text { e } \quad B_{i}^{j}=\partial_{i}\left(\pi^{j} \circ \psi\right), \quad i, j=1, \ldots, m .
$$

Então

$$
\tilde{g}_{i j}=A_{i}^{s} A_{j}^{r} g_{r s} .
$$

Assim $|\tilde{g}|=|A|^{2}|g|$. Portanto, sobre $\Omega \cap \Lambda$

$$
\tilde{v}_{g}=\sqrt{\operatorname{det}\left(\tilde{g}_{i j}\right)} d y^{1} \wedge d y^{2} \wedge \cdots \wedge d y^{m}=|A| \sqrt{\operatorname{det}\left(g_{i j}\right)}|B| d x^{1} \wedge d x^{2} \wedge \cdots \wedge d x^{m}=v_{g},
$$

pois $|A|>0$ e $|A||B|=1$.

Uma família de abertos $\left\{\Omega_{i}\right\}_{i \in I}$ com $M=\cup_{i \in I} \Omega_{i}$ é localmente finita se todo ponto $p \in M$ possui uma vizinhança $\Omega$ tal que $\Omega \cap \Omega_{i} \neq \emptyset, i \in I$, apenas para um número finito de índices. $\mathrm{O}$ suporte de uma função $f: M \longrightarrow \mathbb{R}$, denotado por supp $f$, é o fecho do conjunto dos pontos onde $f$ é diferente de zero. Dado um atlas de $M,\left\{\left(\Omega_{i}, \varphi_{i}\right)_{i \in I}\right\}$, diremos que a família $\left(\Omega_{j}, \varphi_{j}, \alpha_{j}\right)_{j \in J}$ é uma partição da unidade subordinada ao atlas $\left\{\left(\Omega_{i}, \varphi_{i}\right)_{i \in I}\right\}$ se

a) Para todo $j, \alpha_{j} \geq 0$ e $\operatorname{supp} \alpha_{j} \subset \Omega_{j}$.

b) A família $\left\{\Omega_{j}\right\}_{j \in J}$ é localmente finita.

c) $\sum_{j \in J} \alpha_{j}(p)=1$, para todo $p \in M$.

d) $\left(\Omega_{j}, \varphi_{j}\right)_{j \in J}$ é um atlas de $M$.

Pode se demostrar que dado um atlas qualquer $\left\{\left(\Omega_{i}, \varphi_{i}\right)\right\}_{i \in I}$ de $M$, existe uma partição da unidade $\left(\Omega_{j}, \varphi_{j}, \alpha_{j}\right)_{j \in J}$ subordinada à $\left(\Omega_{i}, \varphi_{i}\right)_{i \in I}$. Seja $(M, g)$ uma variedade Riemanniana orientada, $f: M \longrightarrow \mathbb{R}$ uma função contínua com suporte compacto, e $\left\{\left(\Omega_{i}, \varphi_{i}\right)\right\}_{i \in I}$ um atlas de $M$ compatível com a orietação. Definimos a integral (integral de Lebesgue) de $f$ como

$$
\int_{M} f v_{g}=\sum_{j \in J} \int_{\varphi\left(\Omega_{j}\right)}\left(\alpha_{j} \sqrt{|g|} f\right) \circ \varphi^{-1} d x,
$$

onde $\left(\Omega_{j}, \varphi_{j}, \alpha_{j}\right)_{j \in J}$ é uma partição da unidade subordinada à $\left\{\left(\Omega_{i}, \varphi_{i}\right)\right\}_{i \in I}$, e $d x$ denota o elemento de volume de Lebesgue em $\mathbb{R}^{m}$. Não é difícil provar que tal definição não depende da escolha do atlas $\left\{\left(\Omega_{i}, \varphi_{i}\right)\right\}_{i \in I}$ e a partição da unidade $\left(\Omega_{j}, \varphi_{j}, \alpha_{j}\right)_{j \in J}$. Assim, a aplicação $f \longrightarrow \int_{M} f v_{g}$ define uma medida de Radon positiva e a teoria da integração de Lebesgue pode se aplicar.

\subsubsection{Variedades com bordo}

Agora exprimiremos uma primeira generalização do conceito de variedades, as variedades com bordo. Denotaremos por $\mathcal{H}^{m}$ o semi-espaço superior fechado

$$
\mathcal{H}^{m}=\left\{\left(x^{1}, \ldots, x^{m}\right) \in \mathbb{R}^{m} \mid x^{m} \geq 0\right\},
$$

com a topologia induzida do $\mathbb{R}^{m}$. Os pontos $\left(x^{1}, \ldots, x^{m}\right) \in \mathcal{H}^{m}$ com $x^{m}>0$ são chamados pontos interiores de $\mathcal{H}^{m}$, e os pontos com $x^{m}=0$ são chamados pontos do bordo de $\mathcal{H}^{m}$. Estes dois conjuntos serão denotados por $\mathcal{H}^{m}$ e $\partial \mathcal{H}^{m}$, respectivamente.

Uma variedade topológica $m$-dimensional $M$ com bordo é um espaço topológico Haussdorff, segundo contável tal que cada ponto possui uma vizinhança aberta homeomorfa à um subconjunto aberto de $\mathcal{H}^{m}$. O conceito de atlas de classe $C^{k}$ se define de forma análoga ao caso das variedades $m$-dimensionais. Uma variedade $m$-dimensional com bordo de classe $C^{k}, k \in \mathbb{N}$, é uma variedade topológica $m$-dimensional munida de um atlas $C^{k}$-completo. Um ponto $p \in M$ é chamado um ponto interior se para alguma carta $(\Omega, \varphi)$, o ponto $\varphi(p)$ é um ponto interior de $\mathcal{H}^{m}$. Similarmente, 
$p$ é um ponto do bordo de $M$ se $\varphi(p)$ é um ponto do bordo de $\mathcal{H}^{m}$. Pode se mostrar que os conceitos de ponto interior e ponto do bordo de $M$ são bem definidos. Detoremos o conjunto dos pontos do bordo de $M$ por $\partial M$ e por $\stackrel{\circ}{M}$ o conjunto dos pontos interiores. Note que $\stackrel{\circ}{M}=M-\partial M$.

Proposição 2.2. Seja $M$ uma variedade $m$-dimensional com bordo de classe $C^{k}$. Então $\partial M$ é uma variedade (sem bordo) $(m-1)$-dimensional de classe $C^{k}$. Além disso, $\partial M$ é orientável se $M$ é orientável.

Dada uma variedade com bordo $M$ e $p \in \partial M$. Dizemos que um vetor tangente $X \in T_{p} M$ é um vetor interior se $X_{p} \notin T_{p} \partial M$ e existe um número real positivo $\epsilon$ e uma curva $c:[0, \epsilon[\longrightarrow M$ tal que $c(0)=p, c(] 0, \epsilon[) \subset \stackrel{\circ}{ }$, e $c^{\prime}(0)=X_{p}$. Um vetor $X_{p} \in T_{p} M$ é dito exterior se $-X_{p}$ é interior. Um campo de vetores ao longo do bordo $\partial M$ é uma função $X: \partial M \longrightarrow T M$ tal que $X(p) \in T_{p} M$, para todo $p \in \partial M$. Um campo de vetores ao longo do bordo é dito tangente se $X_{p} \in T_{p} \partial M \subset T_{p} M$, para todo $p \in \partial M$. Um campo de vetores ao longo do bordo é chamado exterior (interior) se para todo $p \in \partial M, X_{p}$ é um vetor exterior (interior).

Seja $(M, g)$ uma variedade Riemanniana orientável com bordo. A restrição da métrica $g$ ao bordo $\partial M$ induz uma métrica Riemanniana sobre $\partial M$, vamos denotar tal métrica também por $g$. Um campo de vetores $X$ ao longo do bordo é dito normal, com respeito à $g$, se $g(X(p), V)=0$ para todo $p \in \partial M$ e todo $V \in T_{p} \partial M$. Um campo de vetores $X$ é chamado unitário se $X(p)$ tem comprimento um, para todo $p$.

Proposição 2.3. Seja $(M, g)$ uma variedade Riemanniana orientada, com bordo. Então existe um único campo vetorial normal unitário exterior ao longo do bordo.

Dada a variedade Riemanniana orientada $(M, g)$, denotaremos por $\eta^{g}$ o campo normal unitário exterior à $\partial M$, com respeito à $g$. Quando não houver risco de confusão usaremos $\eta$ no lugar de $\eta^{g}$.

Seja $(M, g)$ uma variedade Riemanniana compacta orientada com bordo, e $f: M \longrightarrow \mathbb{R}$ uma função de classe $C^{2}$. O Laplaciano $\Delta_{g} f$ de $f$ é definido por

$$
\Delta_{g} f=-t r_{g} \nabla^{2} f=-t r_{g} \operatorname{Hess}_{f} .
$$

Numa carta coordenada $(\Omega, \varphi)$ temos

$$
\Delta_{g} f=-g^{i j}\left(\partial_{i j} f-\Gamma_{i j}^{s} \partial_{s} f\right)
$$

Dadas $u, v \in C^{2}(M)$, temos a seguinte fórmula de integração por partes

$$
\int_{M} v \Delta_{g} u v_{g}=\int_{M}\langle\nabla u, \nabla v\rangle v_{g}-\int_{\partial M} v \frac{\partial u}{\partial \eta} \sigma_{g}
$$

onde $\sigma_{g}$ denota a $(m-1)$-forma de volume sobre $\partial M$ induzida por $g,\langle$,$\rangle é o produto interno para$ 1 -formas induzido por $g$, e a derivada normal exterior de $u, \frac{\partial u}{\partial \eta}$, é definida por

$$
\frac{\partial u}{\partial \eta}(p)=d u_{p}(\eta(p)), \quad \text { para cada } p \in \partial M .
$$

A segunda forma é o $(2,0)$-tensor simétrico definido por

$$
I I_{g}(X, Y)=g\left(\eta, \nabla_{X} Y\right), \quad X, Y \in \mathfrak{X}(\partial M) .
$$

A curvatura média $H_{g}$ do bordo $\partial M$, calculada respeito à normal interior, é definida como sendo

$$
H_{g}=-\frac{1}{m-1} \operatorname{tr}_{g} I I_{g}
$$

Em coordenadas locais,

$$
H_{g}=-\frac{1}{m-1} g^{\alpha \beta} I I_{\alpha \beta}, \quad I I_{\alpha \beta}=I I\left(\partial_{\alpha}, \partial_{\beta}\right) \quad \text { e } \quad \alpha, \beta=1, \ldots, m-1 .
$$




\subsubsection{Mudanças conforme}

Nesta seção vamos estabelecer a relação entre as curvaturas de duas métricas conformes. Relembremos que:

Definição 2.1. Duas métricas Riemannianas sobre $M, \tilde{g}$ e $g$, são ditas conforme se existe uma função positiva $\varphi \in C^{\infty}(M)$ tal que

$$
\tilde{g}=\varphi^{\frac{4}{m-2}} g .
$$

A classe conforme $[g]$ de $g$ é a classe de todas as métricas conformes à $g$.

Seja $M$ uma variedade $m$-dimensional, no restante desta seção $g$ denotará uma métrica Riemanniana sobre $M$ e $\tilde{g}$ uma métrica conforme à $g$, como em (2.12). A seguinte proposição estabelece a relação entre as conexões Riemannianas de $\tilde{g}$ e $g$.

Proposição 2.4. Dados $X, Y \in \mathfrak{X}(M)$ temos

$$
\nabla_{X}^{\tilde{g}} Y=\nabla_{X}^{g} Y+\frac{2}{m-2} \varphi^{-1}\left(d \varphi(X) Y+d \varphi(Y) X-g(X, Y) \operatorname{grad}_{g} \varphi\right),
$$

onde $\operatorname{grad}_{g} \varphi$ denota o campo de vetores dual, com respeito à $g$, da 1 -forma $d \varphi$. Isto é, $\operatorname{grad}_{g} \varphi$ é o campo de vetores definido por

$$
d \varphi_{p}(X)=g\left(\operatorname{grad}_{g} \varphi(p), X\right), \quad p \in M, X \in T_{p} M .
$$

$\operatorname{grad}_{g} \varphi$ é chamado o gradiente com respeito à $g$ de $\varphi$.

Demonstração. Denotaremos por $\tilde{\Gamma}_{i j}^{l}$ e $\Gamma_{i j}^{l}$ os símbolos de Christoffel das conexões $\nabla^{\tilde{g}}$ e $\nabla^{g}$, respectivamente. Então, num sistema coordenado, da equação (2.3) temos

$$
\begin{aligned}
2 \tilde{\Gamma}_{i j}^{l}= & \tilde{g}^{l s}\left(\partial_{j} \tilde{g}_{i s}+\partial_{i} \tilde{g}_{s j}-\partial_{s} \tilde{g}_{i j}\right) \\
= & \varphi^{-\frac{4}{m-2}} g^{l s}\left(\partial_{j} \varphi^{\frac{4}{m-2}} g_{i s}+\partial_{i} \varphi^{\frac{4}{m-2}} g_{s j}-\partial_{s} \varphi^{\frac{4}{m-2}} g_{i j}\right) \\
= & \varphi^{-\frac{4}{m-2}} g^{l s}\left\{\frac{4}{m-2} \varphi^{\frac{4}{m-2}-1}\left(\left(\partial_{j} \varphi\right) g_{i s}+\left(\partial_{i} \varphi\right) g_{s j}-\left(\partial_{s} \varphi\right) g_{i j}\right)\right. \\
& \left.\quad+\varphi^{\frac{4}{m-2}}\left(\partial_{j} g_{i s}+\partial_{i} g_{s j}-\partial_{s} g_{i j}\right)\right\} \\
= & 2 \Gamma_{i j}^{l}+\frac{4}{m-2} \varphi^{-1}\left(\delta_{i}^{l} \partial_{j} \varphi+\delta_{j}^{l} \partial_{i} \varphi-g_{i j}\left(\operatorname{grad}_{g}\right)^{l} \varphi\right),
\end{aligned}
$$

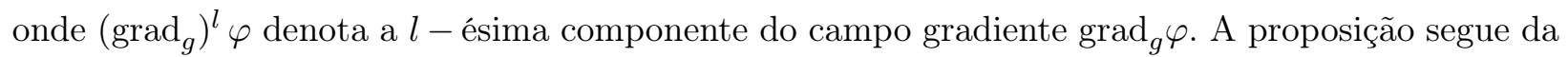
equação acima.

A proposição seguinte fornece as fórmulas para calcular as componentes do tensor de Ricci, $\operatorname{Ric}_{\tilde{g}}$, em termos das componentes do tensor $\operatorname{Ric}_{g}$.

Proposição 2.5. Sejam $1 \leq i, j \leq m$. As componentes $\tilde{R}_{i j}$ do tensor de Ricci, $\operatorname{Ric}_{\tilde{g}}$, são dadas por

$$
\tilde{R}_{i j}=R_{i j}+\frac{2}{m-2} \varphi^{-2}\left((m-4) \partial_{i} \varphi \partial_{j} \varphi-(m-2) \varphi \partial_{i} \partial_{j} \varphi+\left(\varphi \Delta_{g} \varphi+\left|\operatorname{grad}_{g} \varphi\right|^{2}\right) g_{i j}\right),
$$

onde as $R_{i j}$ denotam as componentes do tensor $\mathrm{Ric}_{g}$.

Demonstração. dado um ponto $p \in M$ e um sistema de coordenadas normais, com respeito à métrica $\tilde{g}$, de $M$ em $p$, temos que

$$
\tilde{R}_{i j}=\partial_{k} \tilde{\Gamma}_{i j}^{k}-\partial_{i} \tilde{\Gamma}_{j k}^{k},
$$


Da equação (2.14) segue

$$
\begin{aligned}
\partial_{k} \tilde{\Gamma}_{i j}^{k}= & \partial_{k}\left(\Gamma_{i j}^{k}+\frac{2}{m-2} \varphi^{-1}\left(\delta_{j}^{k} \partial_{i} \varphi+\delta_{i}^{k} \partial_{j} \varphi-g_{i j} \operatorname{grad}^{k} \varphi\right)\right) \\
= & \partial_{k} \Gamma_{i j}^{k}+\frac{2}{m-2} \partial_{k} \varphi^{-1}\left(\delta_{j}^{k} \partial_{i} \varphi+\delta_{i}^{k} \partial_{j} \varphi-g_{i j} \operatorname{grad}^{k} \varphi\right) \\
= & \partial_{k} \Gamma_{i j}^{k}-\frac{2}{m-2} \varphi^{-2}\left(\partial_{k} \varphi\right)\left(\delta_{j}^{k} \partial_{i} \varphi+\delta_{i}^{k} \partial_{j} \varphi-g_{i j} \operatorname{grad}^{k} \varphi\right) \\
& \left.\quad+\frac{2}{m-2} \varphi^{-1}\left(\delta_{i}^{k} \partial_{k} \partial_{j} \varphi+\delta_{j}^{k} \partial_{k} \partial_{i} \varphi+\frac{4}{m-2} \varphi^{-1} g_{i j}\left(\partial_{k} \varphi\right) g^{k s} \partial_{k} \partial_{s} \varphi\right)\right) \\
= & \partial_{k} \Gamma_{i j}^{k}-\frac{2}{m-2} \varphi^{-2}\left(2 \partial_{j} \varphi \partial_{i} \varphi-\partial_{k} \varphi\left(\operatorname{grad}^{k} \varphi\right)\right)+\frac{2}{m-2} \varphi^{-1}\left(2 \partial_{j} \partial_{i} \varphi-g_{i j} g^{k k} \partial_{k} \partial_{k} \varphi\right)
\end{aligned}
$$

onde na terceira e quarta igualdade temos usado que (em $p)$

$$
\partial_{\gamma} \tilde{g}_{i j}=0, \quad \partial_{\gamma} g_{i j}=-\frac{4}{m-2} \varphi^{-1} g_{i j} \partial_{\gamma} \varphi, \quad \text { e } \quad \partial_{\gamma} g^{i j}=-g^{i r} g^{j s} \partial_{\gamma} g_{s r}=\frac{4}{m-2} \varphi^{-1} g^{i j} \partial_{\gamma} \varphi,
$$

para $i, j, \gamma=1, \ldots, m$. Além disso,

$$
g^{i j}=g_{i j}=0, j \neq i \text {. }
$$

Também (em $p)$

$$
\begin{aligned}
\partial_{i} \tilde{\Gamma}_{j k}^{k} & =\partial_{i}\left(\Gamma_{j k}^{k}+\frac{2}{m-2}\left(\delta k^{k} \partial_{j} \varphi+\delta k^{j} \partial_{k} \varphi-g_{k j}\left(\operatorname{grad}^{k} \varphi\right)\right)\right) \\
& =\partial_{i} \Gamma_{j k}^{k}+\frac{2 m}{m-2} \partial_{i}\left(\varphi^{-1} \partial_{j} \varphi\right) \\
& =\partial_{i} \Gamma_{j k}^{k}-\frac{2 m}{m-2} \varphi^{-2} \partial_{i} \varphi \partial_{j} \varphi+\frac{2 m}{m-2} \varphi^{-1} \partial_{i} \partial_{j} \varphi
\end{aligned}
$$

Logo

$$
\begin{aligned}
\left(\partial_{k} \tilde{\Gamma}_{i j}^{k}-\partial_{i} \tilde{\Gamma}_{j k}^{k}\right)=\left(\partial_{k} \Gamma_{j i}^{k}-\partial_{i} \Gamma_{j k}^{k}\right)+2 \varphi^{-2}\left(\partial_{i} \varphi \partial_{j} \varphi+\frac{1}{m-2} g_{i j}\left|\operatorname{grad}_{g} \varphi\right|^{2}\right) \\
-2 \varphi^{-1}\left(\partial_{i} \partial_{j} \varphi+\frac{1}{m-2} g_{i j} g^{k k} \partial_{k} \partial_{k} \varphi\right) .
\end{aligned}
$$

Agora, como $\tilde{\Gamma}_{s t}^{r}=0($ em $p)$, então

$$
\Gamma_{s t}^{r}=-\frac{2}{m-2} \varphi^{-1}\left(\delta_{t}^{r} \partial_{s} \varphi+\delta_{s}^{r} \partial_{t} \varphi-g_{s t} \operatorname{grad}^{r} \varphi\right) .
$$

Logo,

$$
\Gamma_{k l}^{k} \Gamma_{i j}^{l}=\frac{4 m}{(m-2)^{2}} \varphi^{-2}\left(2 \partial_{i} \varphi \partial_{j} \varphi-g_{i j}\left|\operatorname{grad}_{g} \varphi\right|^{2}\right)
$$

$\mathrm{e}$

$$
\Gamma_{i l}^{k} \Gamma_{k j}^{l}=\frac{4}{(m-2)^{2}} \varphi^{-2}\left((m+2) \partial_{i} \varphi \partial_{j} \varphi-2 g_{i j}\left|\operatorname{grad}_{g} \varphi\right|^{2}\right) .
$$

Portanto,

$$
\left(\Gamma_{k l}^{k} \Gamma_{i j}^{l}-\Gamma_{i l}^{k} \Gamma_{k j}^{l}\right)=\frac{4}{m-2} \varphi^{-2}\left(\partial_{i} \varphi \partial_{j} \varphi-g_{i j}\left|\operatorname{grad}_{g} \varphi\right|^{2}\right)
$$


Assim, das equações (2.16), (2.17) e (2.18) temos que (em $p)$

$$
\begin{aligned}
\tilde{R}_{i j}= & R_{i j}+\frac{2}{m-2} \varphi^{-2}\left((m-4) \partial_{i} \varphi \partial_{j} \varphi+g_{i j}\left|\operatorname{grad}_{g} \varphi\right|^{2}\right)-\frac{2}{m-2} \varphi^{-1}\left(\partial_{i} \partial_{j} \varphi+g_{i j} g^{k k} \partial_{k} \partial_{k} \varphi\right. \\
& \left.-2 g_{i j} \varphi^{-1}\left|\operatorname{grad}_{g} \varphi\right|^{2}\right) \\
& =R_{i j}+\frac{2}{m-2} \varphi^{-2}\left((m-4) \partial_{i} \varphi \partial_{j} \varphi-(m-2) \varphi \partial_{i} \partial_{j} \varphi+\left(\varphi \Delta_{g} \varphi+\left|\operatorname{grad}_{g} \varphi\right|^{2}\right) g_{i j}\right) .
\end{aligned}
$$

Uma consequência de (2.15) é o seguinte corolário

Corolario 2.1. Sejam $M$ uma variedade $m$-dimensional suave, $\tilde{g}$ e $g$ duas métricas conforme sobre $M, \operatorname{com} \tilde{g}=\varphi^{\frac{4}{m-2}} g$. Então

$$
R_{\tilde{g}}=\frac{4(m-1)}{(m-2)} \varphi^{-\frac{(m+2)}{(m-2)}}\left(\Delta_{g} \varphi+\frac{(m-2)}{4(m-1)} \varphi R_{g}\right) .
$$

Demonstração. De (2.8) e (2.15) temos

$$
\begin{aligned}
R_{\tilde{g}} & =\tilde{g}^{i j} \tilde{R}_{i j} \\
& =\varphi^{-\frac{4}{m-2}} g^{i j}\left(R_{i j}+\frac{2}{m-2} \varphi^{-1} g_{i j} \Delta_{g} \varphi+\frac{2}{m-2} \varphi^{-2}\left((m-4) \partial_{i} \varphi \partial_{j} \varphi-(m-2) \varphi \partial_{j} \partial_{i} \varphi\right.\right. \\
& \left.\left.+g_{i j}\left|\operatorname{grad}_{g} \varphi\right|^{2}\right)\right) \\
& =\varphi^{-\frac{4}{m-2}}\left(R_{g}+\frac{2 m}{m-2} \varphi^{-1} \Delta_{g} \varphi+2 \varphi^{-1} \Delta_{g} \varphi\right) \\
& =\varphi^{-\frac{4}{m-2}}\left(R_{g}+\frac{4(m-1)}{(m-2)} \varphi^{-1} \Delta_{g} \varphi\right) \\
& =\frac{4(m-1)}{(m-2)} \varphi^{-\frac{(m+2)}{(m-2)}}\left(\Delta_{g} \varphi+\frac{(m-2)}{4(m-1)} \varphi R_{g}\right) .
\end{aligned}
$$

Finalmente se $M$ é uma variedade $m$-dimensional com bordo, então podemos expressar a curvatura média do bordo $\partial M$, calculada com respeito à normal interior induzida por $\tilde{g}$ em termos da curvatura media do bordo calculada com respeito à normal induzida por $g$. Com efeito,

Proposição 2.6. Sejam $M$ uma variedade $m$-dimensional suave com bordo, $\tilde{g}$ e $g$ duas métricas conforme sobre $M$, com $\tilde{g}=\varphi^{\frac{4}{m-2}} g$. Então

$$
H_{\tilde{g}}=\frac{2}{(m-2)} \varphi^{\frac{-m}{m-2}}\left(\frac{\partial \varphi}{\partial \eta^{g}}+\frac{(m-2)}{2} \varphi H_{g}\right) .
$$

Demonstração. De (2.11) segue se

$$
H_{\tilde{g}}=-\frac{1}{(m-1)} \operatorname{tr}_{\tilde{g}}\left((X, Y) \longrightarrow \tilde{g}\left(\eta^{\tilde{g}}, \nabla_{X}^{\tilde{g}} Y\right)\right), \quad X, Y \in T \partial M .
$$

Agora, como $1=\tilde{g}\left(\eta^{\tilde{g}}, \eta^{\tilde{g}}\right)=g\left(\varphi^{\frac{2}{m-2}} \eta^{\tilde{g}}, \varphi^{\frac{2}{m-2}} \eta^{\tilde{g}}\right)$, então $\eta^{g}=\varphi^{\frac{2}{m-2}} \eta^{\tilde{g}}$. Logo da equação (2.13) 
temos

$$
\begin{aligned}
H_{\tilde{g}} & =-\frac{1}{m-1} \operatorname{tr}_{\tilde{g}}\left((X, Y) \longrightarrow \tilde{g}\left(\eta^{\tilde{g}}, \nabla_{X}^{\tilde{g}} Y\right)\right) \\
& =-\frac{1}{m-1} \varphi^{\frac{-4}{m-2}} \operatorname{tr}_{g}\left((X, Y) \longrightarrow \varphi^{\frac{2}{m-2}}\left(g\left(\eta^{g}, \nabla_{X}^{g} Y\right)-\frac{2}{m-2} \varphi^{-1} g(X, Y) \frac{\partial \varphi}{\partial \eta^{g}}\right)\right) \\
& =\varphi^{\frac{-2}{m-2}} H_{g}+\frac{2}{(m-2)} \varphi^{\frac{-m}{m-2}} \frac{\partial \varphi}{\partial \eta^{g}} \\
& =\frac{2}{(m-2)} \varphi^{\frac{-m}{m-2}}\left(\frac{\partial \varphi}{\partial \eta^{g}}+\frac{(m-2)}{2} \varphi H_{g}\right) .
\end{aligned}
$$

\subsection{Análise Funcional}

No decorrer desta tese consideraremos espaços vetoriais reais. Sejam E, F espaços de Banach, isto é, espaços vetoriais normados completos. Relembramos que $\mathrm{T} \in \mathcal{L}(\mathrm{E}, \mathrm{F})$ se $\mathrm{T}$ é linear e limitado no seguinte sentido

$$
\sup \left\{\frac{\|\mathrm{T} x\|}{\|x\|} \mid x \in \mathrm{E}, x \neq 0\right\}<\infty .
$$

Note que a norma do numerador é a norma de $\mathrm{F}$ e a normado do denominador é a norma de $\mathrm{E}$. Equivalentemente, $\mathrm{T} \in \mathcal{L}(\mathrm{E}, \mathrm{F})$ se e só se $\mathrm{T}$ é linear e contínuo. Dado $\mathrm{T} \in \mathcal{L}(\mathrm{E}, \mathrm{F})$, denotamos por $\|\mathrm{T}\|$ o número

$$
\|\mathrm{T}\|=\sup \left\{\frac{\|\mathrm{T} x\|}{\|x\|}: x \in \mathrm{E}, x \neq 0\right\} .
$$

Não é difícil provar que (2.21) define uma norma sobre $\mathcal{L}(\mathrm{E}, \mathrm{F})$, chamada norma uniforme ou norma do supremo. Além disso, $\mathcal{L}(\mathrm{E}, \mathrm{F})$ com a norma uniforme é um espaço de Banach. No caso $\mathrm{F}=\mathbb{R}$, chamamos ao espaço $\mathrm{E}^{*}=\mathcal{L}(\mathrm{E}, \mathbb{R})$ espaço dual de $\mathrm{E}$.

Uma função $f: \mathrm{E} \longrightarrow \mathrm{F}$ é diferenciável no ponto $e \in \mathrm{E}$ se existe $\mathrm{T}_{e} \in \mathcal{L}(\mathrm{E}, \mathrm{F})$ tal que

$$
\lim _{h \rightarrow 0} \frac{\left\|f(e+h)-f(e)-\mathrm{T}_{e} h\right\|}{\|h\|}=0 .
$$

Neste caso denotaremos $d f_{e}:=\mathrm{T}_{e}$. Dado um subconjunto aberto $U \subset \mathrm{E}$, diremos que $f$ é diferenciável em $U$ se é diferenciável para todo $e \in U . f \in C^{1}(U, \mathrm{~F})$ se a função $d f: U \longrightarrow \mathcal{L}(\mathrm{E}, \mathrm{F})$ dada por

$$
d f(e)=d f_{e},
$$

é contínua. Se a função $d f: \mathrm{E} \longrightarrow \mathrm{F}$ é diferenciável em $U$, denotaremos por $d^{2} f$ sua diferencial. Isto é, $d^{2} f(e)=d(d f)_{e}, e \in U$. Em geral, dado $k \in \mathbb{N}$, definimos

$$
d^{k+1} f(e):=d\left(d^{k} f\right)_{e}, \quad e \in U,
$$

se $d^{k} f$ é diferenciável em $U$. Dizemos que $f \in C^{k}(U, \mathrm{~F})$ se $d^{k} f$ é contínua. Finalmente, $f \in C^{\infty}(U, \mathrm{~F})$ se $f \in C^{k}(U, \mathrm{~F})$ para todo $k \in \mathbb{N}$.

Se $f \in C^{2}(U, \mathrm{~F})$, então $d^{2} f_{e}, e \in U$, é um operador bilinear simétrico. Isto é,

$$
d^{2} f_{e}(u, w)=d^{2} f_{e}(w, u) \quad \text { para todo } \quad u, w \in \mathrm{E} .
$$

\subsubsection{Teorema da função implícita}

Umas das ferramentas analíticas mais importantes para o estudo das soluções de um problema não linear do tipo

$$
F(x, y)=0
$$


onde $F$ é uma função $F: U \times V \longrightarrow Z$ com $\mathrm{X}, \mathrm{Y}, \mathrm{Z}$ espaços da Banach, $U$ e $V$ subconjuntos abertos de $\mathrm{X}$ e $\mathrm{Y}$, respectivamente, é o seguinte teorema da função implícita

Teorema 2.2. Seja $F \in C^{k}(U \times V, \mathrm{Z}), k \geq 1$, onde $\mathrm{Z}$ é um espaço de Banach, $U$ e $V$ são subconjuntos abertos dos espaços de Banach $\mathrm{X}$ e $\mathrm{Y}$, respectivamente. Suponha que $F\left(x_{0}, y_{0}\right)=0$ e a derivada parcial $D_{y} F\left(x_{0}, y_{0}\right)$ é invertível. Então existem vizinhanças abertas $\Theta$ de $x_{0}$ em $\mathrm{X}$ e $\Lambda$ de $y_{0}$ em $Y$ e uma função $g \in C^{k}(\Theta, \Lambda)$ tal que

i) $F(x, g(x))=0$ para todo $x \in \Theta$,

ii) $F(x, y)=0, \operatorname{com}(x, y) \in \Theta \times \Lambda$, implica $y=g(x)$,

iii) $d g_{x}=-\left[D_{y} F(x, g(x))\right]^{-1} \circ D_{x} F(x, g(x))$ para todo $x \in \Theta$.

\subsubsection{Operadores de Fredholm}

O propósito desta seção é apresentar alguns aspectos básicos dos operadores de Fredholm. Os operadores de Fredholm são uma classe especial de operadores entre espaços de Banach que desempenham um papel importante no nosso trabalho. Dado $\mathrm{T} \in \mathcal{L}(\mathrm{E}, \mathrm{F})$, com $\mathrm{E}, \mathrm{F}$ espaços de Banach, denotamos por KerT e ImT o espaço nulo e a imagem de T. Isto é,

$$
\operatorname{KerT}=\{e \in \mathrm{E}: \mathrm{T} e=0\} \text { e } \operatorname{Im} \mathrm{T}=\{\mathrm{T} e: e \in \mathrm{E}\} .
$$

Se ImT é um subespaço fechado de $F$ então definimos a condimensão de $\operatorname{Im} T$, denotada por Codim $\operatorname{Im} T$, como a dimensão do espaço quociente $\mathrm{F} / \mathrm{ImT}$. Dizemos que o operador $\mathrm{T} \in \mathcal{L}(\mathrm{E}, \mathrm{F})$ é Fredholm se

a) KerT tem dimensão finita,

b) ImT é um subespaço fechado de $T$, de codimensão finita.

Se $\mathrm{T} \in \mathcal{L}(\mathrm{E}, \mathrm{F})$ é Fredholm definimos o índice de $\mathrm{T}$ por

$$
\text { Ind } \mathbf{T}=\operatorname{dim} \operatorname{Ker} T-\operatorname{Codim} \operatorname{Im} T .
$$

Proposição 2.7. Sejam E, F espaços de Banach e $T \in \mathcal{L}(E, F)$ um operador de Fredholm. Então existem um subespaço fechado $V$ de $\mathrm{E}$ e um subespaço finito dimensional $W$ de $\mathrm{F}$ tais que

$$
\mathrm{E}=\operatorname{KerT} \oplus V \quad \text { e } \quad \mathrm{F}=\operatorname{Im} \mathrm{I} \oplus W .
$$

Em particular, o operador sobrejetor $\mathrm{T}: V \longrightarrow \operatorname{Im} \mathrm{T}$ é um isomorfismo.

A composição de operadores Fredholm também é um operador de Fredholm e o índice da composição é a soma dos índices dos operadores. Isto é conhecido como o teorema do índice.

Teorema 2.3 (Teorema do Índice). Sejam E, F, Z espaços de Banach, $T_{1} \in \mathcal{L}(E, F)$ e $T_{2} \in \mathcal{L}(F, Z)$. Então

i) Se $T_{1}$ e $T_{2}$ são operadores de Fredholm então $T_{2} \circ T_{1}$ também é um operador de Fredholm e Ind $T_{2} \circ T_{1}=$ Ind $T_{1}+$ Ind $T_{2}$.

ii) $\mathrm{Se}_{2} \circ \mathrm{T}_{1}$ é um operador de Fredholm, então $T_{1}$ é um operador de Fredholm se e só se $T_{2}$ é um operador de Fredholm.

A seguinte proposição estabelece que uma perturbação compacta de um operador Fredholm segue sendo Fredholm e seu índice não muda.

Proposição 2.8. Seja $T \in \mathcal{L}(E, F)$ um operador de Fredholm. Então para cada operador compacto ${ }^{4}$ $\mathrm{K}: \mathrm{E} \longrightarrow \mathrm{F}$ o operador $\mathrm{T}+\mathrm{K}$ é Fredholm e Ind $(\mathrm{T}+\mathrm{K})=\operatorname{Ind} \mathrm{T}$.

\footnotetext{
${ }^{4} \mathrm{O}$ operador $\mathrm{K}: \mathrm{E} \longrightarrow \mathrm{F}$ é compacto se a imagem de qualquer conjunto limitado de $\mathrm{E}$, por $\mathrm{K}$, tem fecho compacto em $\mathrm{F}$
} 


\subsubsection{Espaços de Sobolev sobre variedades Riemannianas}

Nesta seção introduzimos os espaços de Sobolev sobre uma variedade Riemanniana e apresentaremos alguns fatos elementares sobre estes espaços.

Seja $(M, g)$ uma variedade Riemanniana. Para $s$ inteiro não negativo, e $u: M \longrightarrow \mathbb{R}$ suave, denotaremos por $\left|\nabla^{s} u\right|$ a norma da sua $s$-ésima derivada covariante $\nabla^{s} u$, induzida pelo produto interno sobre $\Gamma^{s}(M)=\Gamma_{0}^{s}(M)$. Numa carta de $M$, de (2.2) temos

$$
\left|\nabla^{s} u\right|^{2}=g^{i_{1} j_{1}} \cdots g^{i_{s} j_{s}}\left(\nabla^{s} u\right)_{i_{1} \ldots i_{s}}\left(\nabla^{s} u\right)_{j_{1} \ldots j_{s}} .
$$

Dado um inteiro não negativo $s$, e $p \geq 1$ real, seja

$$
\mathfrak{C}_{s}^{p}(M)=\left\{\left.u \in C^{\infty}(M)\left|\int_{M}\right| \nabla^{j} u\right|^{p} v_{g}<\infty, j=0, \ldots, s\right\} .
$$

Note que quando $M$ é compacta, então $\mathfrak{C}_{s}^{p}(M)=C^{\infty}(M)$, para qualquer $s$ e qualquer $p \geq 1$. Para $u \in \mathfrak{C}_{s}^{p}(M)$ definimos

$$
\|u\|_{\mathrm{H}_{p}^{s}}=\sum_{j=0}^{s}\left(\int_{M}\left|\nabla^{j} u\right|^{p} v_{g}\right)^{1 / p} .
$$

Dada uma variedade Riemanniana $(M, g)$, um inteiro não negativo $s$, e $p \geq 1$ real, o espaço de Sobolev $\mathrm{H}_{p}^{s}(M)$ é a completação de $\mathfrak{C}_{p}^{s}(M)$ com respeito à $\|\cdot\|_{\mathrm{H}_{p}^{s}}$. Observa que $\mathrm{H}_{p}^{0}(M)=\mathrm{L}_{p}(M)$.

$\mathrm{Na}$ definição do espaço $\mathrm{H}_{p}^{s}(M)$, de fato, podemos usar outras normas equivalentes. Por exemplo, poderíamos usar

$$
\left(\sum_{j=0}^{s} \int_{M}\left|\nabla^{j} u\right|^{p} v_{g}\right)^{1 / p} .
$$

Quando $p=2, \mathrm{H}_{2}^{s}(M)$ é um espaço de Hilbert ${ }^{5}$, e a norma (2.23) é induzida pelo produto interno

$$
\langle u, v\rangle=\sum_{j=0}^{s} \int_{M}\left\langle\nabla^{j} u, \nabla^{j} v\right\rangle v_{g} .
$$

Por simplicidade escreveremos $\mathrm{H}^{s}(M)$ para o espaço de Hilbert $\mathrm{H}_{2}^{s}(M)$.

$\mathrm{Na}$ definição do espạ $\mathrm{H}_{p}^{s}(M)$ fazemos uso da estrutura Riemanniana sobre $M$, consequentemente, espera-se que uma mudança de métrica altere este espaço. Mas, para variedades compactas temos

Proposição 2.9. Seja $M$ uma variedade compacta. Então $\mathrm{H}_{p}^{s}(M)$ independe da métrica.

Seja $\mathfrak{D}(M)$ o espaço das funções $u \in C^{\infty}(M)$ com suporte compacto em $M$.

Teorema 2.4. Seja $(M, g)$ uma variedade Riemanniana completa. Então $\mathfrak{D}(M)$ é denso em $\mathrm{H}_{p}^{1}(M)$ para qualquer $p \geq 1$.

Agora exporemos os teoremas de inclusão de Sobolev para variedades compactas.

Teorema 2.5 (Teorema de inclusão de Sobolev). Seja $(M, g)$ uma variedade Riemanniana $m$-dimensional, compacta. Então, para quaisquer dois números reais $1 \leq q<p$ e quaisquer dois inteiros $0 \leq r<s$

i) Se $\frac{1}{p}=\frac{1}{q}-\frac{(s-r)}{m}$, então $\mathrm{H}_{q}^{s}(M)$ é incluído continuamente ${ }^{6}$ em $\mathrm{H}_{p}^{r}(M)$. Em particular, para qualquer real $q \in\left[0, m\left[, \mathrm{H}_{q}^{1}(M)\right.\right.$ é imerso continuamente em $\mathrm{L}_{p}(M)$, com $\frac{1}{p}=\frac{1}{q}-\frac{1}{m}$.

\footnotetext{
${ }^{5} \mathrm{Um}$ espaço de Hilbert $\mathrm{H}$ é um espaço vetorial munido de um produto interno $\langle\cdot, \cdot\rangle$, tal que $\mathrm{H}$ é completo com respeito à norma $|h|^{2}=\langle h, h\rangle, h \in \mathrm{H}$.

${ }^{6}$ Dizemos que o espaço vetorial normado $\left(\mathrm{E},|\cdot|_{\mathrm{E}}\right)$ é incluído continuamente no espaço vetorial normado $\left(\mathrm{F},|\cdot|_{\mathrm{F}}\right)$ se $\mathrm{E} \subset \mathrm{F}$ e existe uma constante $C>0$ tal que $|x|_{\mathrm{F}} \leq C|x|_{\mathrm{E}}$ para todo $x \in \mathrm{E}$.
} 
ii) Se $\frac{1}{q}<\frac{(s-r)}{m}$, então $\mathrm{H}_{q}^{s}(M)$ é incluído continuamente em $C^{r}(M)$.

iii) Se $\frac{1}{q} \leq \frac{1-\alpha}{m}, \alpha \in(0,1)$, então $\mathrm{H}_{q}^{1}(M)$ é incluído continuamente em $C^{\alpha}(M)$, onde $C^{\alpha}(M)$ denota o espaço das funções contínuas $u: M \longrightarrow \mathbb{R}$ para as quais a norma

$$
\|u\|_{C^{\alpha}}=\max _{x \in M}|u(x)|+\max _{x \neq y \in M} \frac{|u(y)-u(x)|}{d_{g}(x, y)^{\alpha}},
$$

é finita. Aqui $d_{g}$ denota a distância associada a $g$.

Agora discutimos a propriedade de compacidade das inclusões de Sobolev. Dados dois espaços vetoriais normados $\left(E,|\cdot|_{E}\right)$ e $\left(F,|\cdot|_{F}\right)$, com $E \subset F$, relembramos que a inclusão de $E$ em $F$ é dita compacta se subconjuntos limitados de $\left(\mathrm{E},|\cdot|_{\mathrm{E}}\right)$ têm fecho compacto em $\left(\mathrm{F},|\cdot|_{\mathrm{F}}\right)$. Isto implica que sequências limitadas em $(E,|\cdot| E)$ têm subsequências convergentes em $\left(F,|\cdot|_{F}\right)$. Claramente, se a inclusão de $\mathrm{E}$ em $\mathrm{F}$ é compacta, então também é contínua.

Teorema 2.6 (Teorema de Rellich-Kondrakov). Seja $(M, g)$ uma variedade Riemanniana $m$-dimensional compacta.

i) Para quaisquer inteiros $r \geq 0$ e $s \geq 1$, e quaisquer números reais $q \geq 1$ e $1 \leq p<\frac{m q}{m-s q}$, a inclusão de $\mathrm{H}_{q}^{r+s}(M)$ em $\mathrm{H}_{p}^{r}(M)$ é compacta. Em particular, para qualquer real $q \in[1, m[\mathrm{e}$ qualquer $p \geq 1$ tal que $\frac{1}{p}>\frac{1}{q}-\frac{1}{m}$, a inclusão de $\mathrm{H}_{q}^{1}(M)$ em $\mathrm{L}_{p}(M)$ é compacta.

ii) Para qualquer $q>m$, e qualquer $\alpha \in] 0,1\left[\right.$ tal que $(1-\alpha) q>m$, a inclusão de $\mathrm{H}_{q}^{1}(M)$ em $C^{\alpha}(M)$ é compacta. Em particular, a inclusão de $\mathrm{H}_{q}^{1}(M)$ em $C^{0}(M)$ é compacta.

Existe uma versão dos teoremas (2.5) e (2.6) para variedade com bordo. Mais precisamente;

Teorema 2.7. Seja $M$ uma variedade $m$-dimesnional compacta com bordo. Então

i) Se $\frac{1}{p} \geq \frac{1}{q}-\frac{(s-r)}{m}>0$, então $\mathrm{H}_{q}^{s}(M)$ é incluído continuamente em $\mathrm{H}_{p}^{r}(M)$. Em particular, se $\frac{1}{p} \geq \frac{1}{q}-\frac{s}{m}>0$, então $\mathrm{H}_{q}^{s}(M)$ é incluído continuamente em $\mathrm{L}_{p}(M)$.

ii) Se $\frac{1}{t} \geq \frac{1}{m-1}\left(\frac{m}{q}-s\right)$, então $\mathrm{H}_{q}^{s}(M)$ é incluído continuamente em $\mathrm{L}_{t}(\partial M)$.

iii) Suponha que é certa a desigualdade estrita em (i) e (ii). Então as inclusões de $\mathrm{H}_{q}^{s}(M)$ em $\mathrm{H}_{p}^{r}(M)$ e $\mathrm{L}_{t}(\partial M)$, respectivamente, são compactas. Além disso, as seguintes inclusões são contínuas:

iv) $\mathrm{H}_{q}^{s}(M) \subset C^{r}(\bar{M})$, se $s-\frac{m}{q}>r, r$ inteiro. Aqui $C^{r}(\bar{M})$ denota as funções de classe $C^{r}$ até o bordo.

v) $\mathrm{H}_{p}^{s}(M) \subset C^{\alpha}(\bar{M})$, se $0<\alpha<1$, e $m \leq p(s-\alpha)$.

Finalmente queremos exprimir que os espaços de Sobolev $\mathrm{H}_{p}^{s}(M)$ podem ser generalizados para o caso em que $s$ é um real qualquer. Para uma definição e propriedades dos espaços $\mathrm{H}^{s}(M)$ com $s$ real, pode se consultar o capítulo 4 em [Taylor , 2011a] ou capítulo 1 em [Lions e Magenes , 1972]. Para o caso do espaço $\mathrm{H}_{p}^{s}(M)$, com $p$ inteiro positivo e $s$ real sugerimos ao leitor as referências citadas em [Lions e Magenes, 1972].

Seja $(M, g)$ uma variedade Riemanniana compacta com bordo. Dada $u \in C^{1}(\bar{M})$ podemos definir

$$
\gamma u=\left.u\right|_{\partial M}
$$

Teorema 2.8 (Teorema do traço). Para todo $s>\frac{1}{2}$, existe um único operador contínuo

$$
\gamma: \mathrm{H}^{s}(M) \longrightarrow \mathrm{H}^{s-\frac{1}{2}}(\partial M)
$$

tal que $\gamma u=\left.u\right|_{\partial M}$ para toda $u \in C^{1}(\bar{M})$. 


\subsubsection{Variedades de Banach}

Falamos das variedades com bordo como uma generalização do conceito de variedade, agora exporemos outro tipo de generalização: as variedades de Banach.

Sejam E um espaço de Banach, $M$ um espaço topológico. Dizemos que $M$ é uma variedade de Banach de classe $C^{k}$, modelada por E, se existe um recobrimento aberto $\left\{U_{\alpha}\right\}$ de $M$ e uma família de funções $\left\{\varphi_{\alpha}\right\}$ com as seguintes propriedades:

i) $\varphi_{\alpha}\left(U_{\alpha}\right)$ é aberto em E e $\varphi_{\alpha}: U_{\alpha} \longrightarrow \varphi_{\alpha}\left(U_{\alpha}\right)$ é um homeomorfismo.

ii) Se $U_{\alpha} \cap U_{\beta} \neq \emptyset$, então a função de transição

$$
\varphi_{\alpha} \circ \varphi_{\beta}^{-1}: \varphi_{\beta}\left(U_{\alpha} \cap U_{\beta}\right) \longrightarrow \varphi_{\alpha}\left(U_{\alpha} \cap U_{\beta}\right)
$$

é de classe $C^{k}$.

Os pares $\left(U_{\alpha}, \varphi_{\alpha}\right)$ são chamados cartas de $M$, e a família $\left\{\left(U_{\alpha}, \varphi_{\alpha}\right)\right\}$ um atlas de classe $C^{k}$. Diremos que $M$ é uma variedade de Banach suave ou simplesmente uma variedade de Banach se as função de transição são de classe $C^{\infty}$. Os conceitos de diferenciabilidade, vetor tangente, e espaço tangente, definidos anteriormente, se definem análogamente no caso de variedades de Banach. Uma atenção especial deve ser dada à definição de subvariedades, devido ao fato que subespaços fechados de um espaço de Banach de dimensão infinita podem não ter complementar fechado no espaço.

Sejam $M$ uma variedade de Banach modelada no espaço de Banach $\mathrm{E}_{\text {e }} \mathrm{E}_{i}, i=1,2$, subespaços de Banach de $\mathrm{E}$ tais que $\mathrm{E}=\mathrm{E}_{1} \oplus \mathrm{E}_{2}$. Um subconjunto $N \subset M$ é chamado uma subvariedade de Banach de $M$, se para todo $p \in N$, existe uma carta $(U, \varphi)$ tal que $\varphi$ dá um isomorfismo de $U$ com um produto $U_{1} \times U_{2} \subset \mathrm{E}_{1} \times \mathrm{E}_{2}$, com $U_{i}$ aberto em $\mathrm{E}_{i}, i=1$, 2 , de tal forma que

$$
\varphi(U \cap N)=U_{1} \times\left\{e_{2}\right\},
$$

para algum $e_{2} \in \mathrm{E}_{2}$. Claramente, se $\pi_{1}: \mathrm{E}_{1} \times \mathrm{E}_{2} \longrightarrow \mathrm{E}_{1}$ denota a projeção sobre o primeiro fator, então $\left(U \cap N, \pi_{1} \circ \varphi\right)$ é uma carta de $N$. Isto permite dar à $N$ uma estrutura de variedade de Banach modelada por $E_{1}$. Com esta estrutura temos:

Proposição 2.10. Seja $f: M \longrightarrow Z$ uma função entre variedades de Banach suaves. Se $N \subset Z$ é uma subvariedade de $Z$ tal que $f(M) \subset N$, então $f$ é diferenciável se e só se a função $\tilde{f}: M \longrightarrow N$, dada por $\tilde{f}(p)=f(p), p \in M$, é diferenciável.

Exporemos agora dois critérios para construir subvariedades. Precisaremos de algumas definições prévias. Uma função $f: M \longrightarrow Z$ de classe $C^{k}$ entre variedades de Banach é dita uma submersão se para todo $p \in M$ a diferencial $d f_{p}: T_{p} M \longrightarrow T_{f(p)} Z$ é sobrejetora e seu kernel, Ker $d f_{p}$, é complementado em $T_{p} M . f$ é chamada uma imersão se para todo $p \in M$ a diferencial $d f_{p}$ é injetora e $\operatorname{Im} d f_{p}$ é um subespaço fechado e complementado de $T_{f(p)} Z$.

Proposição 2.11. Seja $f: M \longrightarrow Z$ uma função de classe $C^{k}$ sobre variedades de Banach suaves.

i) Se $f$ é uma submersão, então para cada $z \in Z, N=f^{-1}(z)$ é uma subvariedade de $M$ de classe $C^{k}$. Além disso, para todo $p \in N, T_{p} N \simeq \operatorname{Ker} d f_{p}$.

ii) Se $f$ é uma imersão, então para todo $p \in M$ existe um aberto $U \subset M$, com $p \in U$, tal que $f(U) \subset Z$ é uma subvariedade de $Z$ de classe $C^{k}$.

Sejam $M$ e $Z$ variedades de Banach suaves, um fibrado sobre $M$ com fibra (típica) $Z$ é uma variedade de Banach suave $W$ junto com uma função suave $\pi: W \longrightarrow M$, satisfazendo a condição de trivialização local: Para cada $p \in M$ existe um aberto $U \subset M$, com $p \in U$, e um difeomorfismo $\phi: \pi^{-1}(U) \longrightarrow U \times Z$ (chamado uma trivialização local) tal que $\pi=\pi_{1} \circ \phi$, onde $\pi_{1}: U \times Z \longrightarrow U$ é a projeção sobre o primeiro fator, isto é, $\pi_{1}(x, z)=x,(x, z) \in U \times Z$. A fibra em $p$, denotada por $W_{p}$, é o conjunto $\pi^{-1}(p)$ o qual é difeomorfo à $Z$ para cada $p \in M$. 
Embora, localmente, um fibrado $W$ seja um produto, $U \times Z$, isto pode não ser verdade globalmente. O espaço $W$ é chamado espaço total, $M$ o espaço base e $\pi$ a projeção. Às vezes podemos nos referir a um fibrado dizendo: Seja $\pi: W \longrightarrow M$ um fibrado suave. Um tipo especial de fibrados são os fibrados vetoriais no qual a fibra $Z$ é um espaço de Banach e as trivializações locais induzem uma estrutura linear bem definida sobre $W_{p}$ para cada $p \in M$. Mais precisamente temos: Sejam $M$ uma variedade de Banach suave e $\mathrm{E}$ um espaço de Banach, um fibrado vetorial sobre $M$ é um fibrado suave $\pi: W \longrightarrow M$ com fibra $\mathrm{E}$ tal que:

a) Cada fibra $W_{p}$ tem estrutura de espaço de Banach.

b) As trivializações locais $\phi: \pi^{-1}(U) \longrightarrow U \times \mathrm{E}$ são tais que $\left.\pi_{2} \circ \phi\right|_{W_{p}}$ é um isomorfismo linear para cada $p \in M$, onde $\pi_{2}(x, e)=e,(x, e) \in U \times \mathrm{E}$.

c) Dadas duas trivializações $\phi_{i}: \pi^{-1}\left(U_{i}\right) \longrightarrow U_{i} \times \mathrm{E}, i=1,2$, tais que $U_{1} \cap U_{2} \neq \emptyset$, então para cada $p \in U_{1} \cap U_{2}$ a função $\left.\left.\phi_{1}\right|_{W_{p}} \circ \phi_{2}\right|_{W_{p}} ^{-1}: \mathrm{E} \longrightarrow \mathrm{E}$ é um isomorfismos de espaços de Banach, isto é, linear, contínuo e bijetivo. Além disso, a função $\left.\left.U_{1} \cap U_{2} \ni p \longrightarrow \phi_{1}\right|_{W_{p}} \circ \phi_{2}\right|_{W_{p}} ^{-1} \in$ $\mathcal{L}(\mathrm{E}):=\mathcal{L}(\mathrm{E}, \mathrm{E})$ é suave.

O exemplo mais importante de fibrado vetorial é o fibrado tangente $T M=\cup_{p \in M} T_{p} M$. Se $W$ é um fibrado vetorial sobre $M$ o fibrado dual $W^{*}$ é o fibrado cujas fibras são os espaços duais das fibras de $W$ :

$$
W^{*}=\left\{(p, f) \mid f \in W_{p}^{*}\right\} .
$$

Sejam $\pi_{i}: W_{i} \longrightarrow M_{i}$ fibrados suaves sobre $M_{i}, i=1,2$. Um morfismo de fibrados de classe $C^{k}$ é um par de funções $(F, f)$, onde $F: W_{1} \longrightarrow W_{2}$ e $f: M_{1} \longrightarrow M_{2}$ são funções de classe $C^{k}$ tais que $\pi_{2} \circ F=f \circ \pi_{1}$. Quando $M_{1}=M=M_{2}$, diremos que $F: W_{1} \longrightarrow W_{2}$ é um morfismo de fibrados se $\left(F, I d_{M}\right)$ é um morfismo de fibrado. Aqui $I d_{M}$ denota a função identidade sobre $M$.

Sejam $\pi_{1}: W_{1} \longrightarrow M_{1}$ e $\pi_{2}: W_{2} \longrightarrow M_{2}$ fibrados suaves. Dizemos que $\pi_{1}: W_{1} \longrightarrow M_{1}$ é um subfibrado de $\pi_{2}: W_{2} \longrightarrow M_{2}$ se existe um morfismo de fibrados suave $(F, f)$ tal que $F: W_{1} \longrightarrow W_{2}$ e $f: M_{1} \longrightarrow M_{2}$ são imersões.

Finalizamos esta seção expondo un teorema da função implícita sobre fibrados formulado por Lima, Piccione e Zedda em [Lima et al. , 2012]. Dados os fibrados $\pi_{i}: W_{i} \longrightarrow M_{i}, i=1,2$, e um morfismo de fibrados de classe $C^{1}, F: W_{1} \longrightarrow W_{2}$, a derivada vertical de $F$ no ponto $e \in W_{1}$ é a função linear

$$
d_{v e r} F(e): T_{e} W_{1, e} \longrightarrow T_{F(e) W_{2, F(e)}}
$$

dada por a diferencial da restrição $\left.F\right|_{W_{1, e}}: W_{1, e} \longrightarrow W_{2, F(e)}$, onde $W_{1, e}=\pi_{1}^{-1}\left(\pi_{1}(e)\right) \subset W_{1}$ denota a fibra de $W_{1}$ que passa por o ponto $e$, e $W_{2, F(e)}=\pi_{2}^{-1}\left(\pi_{2}(F(e))\right) \subset W_{2}$ é a fibra de $W_{2}$ que passa por $F(e)$.

Proposição 2.12. Sejam $\pi_{i}: W_{i} \longrightarrow M, i=1,2$, fibrados suaves, $F: W_{1} \longrightarrow W_{2}$ um morfismo de fibrados de classe $C^{k}, k \geq 1, s: U \subset M \longrightarrow W_{2}$ uma seção local de classe $C^{k}$, onde $U$ é um subconjunto aberto de $M$ tal que $x_{0} \in U, s\left(x_{0}\right)=e_{2}$, e seja $e_{1} \in F^{-1}\left(e_{2}\right)$. Assuma que a derivada vertical $d_{v e r} F\left(e_{1}\right)$ é um isomorfismo. Então, existem um aberto $V$ em $W_{1} \operatorname{com} e_{1} \in V$ tal que $U^{\prime}=\pi_{1}(V) \subset U$, e uma seção $\tilde{s}: U^{\prime} \longrightarrow W_{1} \operatorname{com} \tilde{s}\left(x_{0}\right)=e_{1}$, tal que $e \in V \cap F^{-1}(s(U))$ se e só se $e \in \tilde{s}\left(U^{\prime}\right)$. 


\section{Capítulo 3}

\section{Teoremas de Bifurcação}

Neste capítulo vamos expor, brevemente, alguns teoremas gerais sobre bifurcação que desempenharam um papel importante na prova do teorema 5.1 o qual é uma das ferramentas principais na obtenção dos objetivos desta tese. As referências básicas deste capítulo são [Lima et al. , 2012], [Smoller e Wasserman , 1990], [Ambrosetti e Prodi , 1993] e [Kielhöfer , 2004].

\subsection{Bifurcação: Aspectos gerais}

A estrutura do conjunto solução de uma equação funcional não linear, em geral, é muito complicada, portanto, torna-se conveniente observar quando é possível obter novas soluções, numa vizinhança de uma dada solução, através de pequenas perturbações. Uma forma de fazer isto, é encontrando ou introduzindo um parâmetro $\tau$ e estudando uma equação da forma

$$
F(\tau, u)=0
$$

a qual possui uma solução fixa para todos os valores do parâmetro. Um fato interessante é quando existe um ramo de novas soluções da equação (3.1) em correspondência com algum valor do parâmetro. Isto é o objeto da teoria da bifurcação. Mais precisamente; dados os espaços de Banach E, Y temos interesse em estudar equações do tipo (3.1), onde $F: \mathbb{R} \times \mathrm{E} \longrightarrow \mathrm{Y}$ é uma função de classe $C^{2}$ tal que

$$
F\left(\tau, u_{0}\right)=0
$$

para todo $\tau \in \mathbb{R}$ e algum $u_{0} \in \mathrm{E}$. Se vale (3.2) temos que para todo $\tau, u=u_{0}$ é uma solução de (3.1). Chamaremos esta de solução trivial. Por simplicidade vamos supor $u_{0}=0$.

Pode acontecer que para algum valor do parâmetro $\tau$ exista uma ou mais soluções de (3.1) as quais ramificam se da solução trivial. Este valor do parâmetro $\tau$ é chamado um instante de bifurcação de (3.1). Mais propriamente, temos a seguinte definição:

Definição 3.1. Dizemos que $\tau^{*}$ é um instante de bifurcação para $F$ (ou da família trivial $\{(\tau, 0)\}_{\tau \in \mathbb{R}}$ ) se existe uma sequência $\left(\tau_{j}, u_{j}\right) \in \mathbb{R} \times \mathrm{E}$, com $u_{j} \neq 0$ e $F\left(\tau_{j}, u_{j}\right)=0$, tal que

$$
\left(\tau_{j}, u_{j}\right) \longrightarrow\left(\tau^{*}, 0\right)
$$

A seguinte proposição é consequência direta do teorema da função implícita

Proposição 3.1. Se $\tau^{*}$ é um instante de bifurcação para $F$, então a derivada parcial $D_{u} F\left(\tau^{*}, 0\right)$ é não invertível.

Quando $E=Y$ e

$$
F(\tau, u)=\tau u-G(u),
$$

então

$$
D_{u} F\left(\tau^{*}, 0\right)=\tau^{*} I-d G_{0} .
$$


Logo da proposição 3.1 temos:

Proposição 3.2. Seja $F$ da forma (3.3). Se $\tau^{*}$ é um instante de bifurcação para $F$, então $\tau^{*}$ pertence ao espectro $\Sigma\left(d G_{0}\right)$ de $d G_{0}$.

Uma pergunta que surge de forma natural é se a recíproca da proposição 3.2 é verdade. Isto é,

Se $\tau^{*} \in \Sigma\left(d G_{0}\right)$, então $\tau^{*}$ é um instante de bifurcação para $F$ ?

A resposta a pergunta acima é negativa. Por exemplo, sejam $\mathrm{E}=\mathbb{R}^{2}=\mathrm{Y}, G: \mathrm{E} \longrightarrow \mathrm{E}$ definida por

$$
G(x, y)=\left(x+y^{3}, y-x^{3}\right) .
$$

Observe que $\tau^{*}=1$ é um autovalor de $d G_{0}=I d_{\mathrm{E}}$, mas não é um instante de bifurcação para

$$
F(x, y)=\tau(x, y)-G(x, y)=\left(\tau x-x-y^{3}, \tau y-y+x^{3}\right) .
$$

Em resumo, o fato de $D_{u} F\left(\tau^{*}, 0\right)$ ser não invertível não garante que $\tau^{*}$ seja um instante de bifurcação para $F$. Diferentes critérios para garantir a existência de instantes de bifurcação encontram se na literatura, mais adiante apresentaremos alguns deles.

\subsubsection{O método de Lyapunov-Schmidt}

A teoria dos operadores de Fredholm pode ser usada para reduzir o problema de dimensão infinita (3.1) à um problema de dimensão finita.

Sejam X,Y,Z espaços de Banach, $U \subset \mathrm{X}, V \subset \mathrm{Y}$ subconjuntos abertos e $F: U \times V \longrightarrow \mathrm{Z}$ uma função tal que $F \in C(U \times V, Z), F\left(x_{0}, y_{0}\right)=0,\left(x_{0}, y_{0}\right) \in U \times V$. Suponha que a função

$$
U \times V \ni(x, y) \longrightarrow D_{x} F(x, y) \in \mathcal{L}(\mathbf{X}, \mathbf{Z}),
$$

é contínua e $D_{x} F\left(\cdot, y_{0}\right)$ é um operador de Fredholm. Então existem subespaços fechados $\mathrm{X}_{0}$ e $\mathrm{Z}_{0}$ em $\mathrm{X}$ e $Z$, respectivamente, tais que

$$
\mathbf{X}=\operatorname{Ker} D_{x} F\left(x_{0}, y_{0}\right) \oplus \mathbf{X}_{0} \quad \text { e } \quad \mathbf{Z}=\operatorname{Im} D_{x} F\left(x_{0}, y_{0}\right) \oplus \mathbf{Z}_{0} .
$$

Esta descomposição dos espaços X e Z permite definir, naturalmente, as projeções

$$
P: \mathrm{X} \longrightarrow \mathrm{N} \quad \text { e } \quad Q: \mathrm{Z} \longrightarrow \mathrm{Z}_{0}
$$

onde $\mathrm{N}=\operatorname{Ker} D_{x} F\left(x_{0}, y_{0}\right)$. O teorema do gráfico fechado garante que as mencionadas projeções são contínuas. Então o seguinte método de redução de Lyapunov-Schmidt é verdade

Teorema 3.1. Existe uma vizinhança aberta $U_{1} \times V_{1}$ de $\left(x_{0}, y_{0}\right)$ em $U \times V \subset \mathrm{X} \times \mathrm{Y}$ tal que o problema

$$
F(x, y)=0 \quad \text { para }(x, y) \in U_{1} \times V_{1},
$$

é equivalente ao problema de dimensão finita

$$
\Phi(v, y)=0 \quad \text { para }(v, y) \in \tilde{U}_{1} \times V_{1} \subset \mathrm{N} \times \mathrm{Y},
$$

onde

$$
\tilde{U}_{1}=P\left(U_{1}\right), \quad v=P(x), \quad x \in U_{1},
$$

e $\Phi: \tilde{U}_{1} \times V_{1} \longrightarrow Z_{0}$ é contínua tal que

$$
\Phi\left(v_{0}, y_{0}\right)=0, \quad v_{0}=P x_{0} .
$$

A função $\Phi$ é chamada uma função de bifurcação. 


\subsubsection{Operadores gradiente}

Os teoremas de bifurcação que usaremos neste trabalho são aplicados sobre operadores gradientes, cuja definição e propriedades básicas exprimimos nesta seção.

Sejam X, Z espaços da Banach, tais que $X$ é continuamente imerso em Z e Z é munido de um produto interno $\langle\cdot, \cdot\rangle$. Uma função contínua $G: U \subset \mathrm{X} \longrightarrow \mathrm{Z}$, onde $U$ é um subconjunto aberto de $\mathrm{X}$, é chamada um operador gradiente, com respeito à produto interno $\langle\cdot, \cdot\rangle$, se existe uma função $g \in C^{1}(U, \mathbb{R})$ tal que

$$
\langle G(x), h\rangle=d g_{x}(h), \quad \text { para todo } x \in U, h \in \mathbf{X} .
$$

A função $g$ é chamada o potencial de $G$. Usaremos a notação $G=\operatorname{grad} g$, alguns autores usam a notação $G=\nabla g$.

Se o operador gradiente $G: U \subset \mathrm{X} \longrightarrow \mathrm{Z}$ é diferenciável, então o potencial $g$ é de classe $C^{2}$. Logo da simetria de $d^{2} g$ segue

Proposição 3.3. Se o operador gradiente $G: U \subset \mathrm{X} \longrightarrow \mathrm{Z}$ é diferenciável, então a sua diferencial $d G_{x}, x \in U$, é simétrica com respeito ao produto interno $\langle\cdot, \cdot\rangle$. Isto é,

$$
\left\langle d G_{x}\left(h_{1}\right), h_{2}\right\rangle=\left\langle h_{1}, d G_{x}\left(h_{2}\right)\right\rangle, \quad \text { para todo } x \in U, h_{1}, h_{2} \in \mathbf{X} .
$$

Impondo certa restrição geométrica ao aberto $U$, pode-se mostrar que a propriedade descrita na proposição acima caracteriza os operadores gradiente. Mais exatamente,

Proposição 3.4. Seja $G: U \longrightarrow \mathrm{Z}$ uma função de classe $C^{1}$ e suponha que o aberto $U \subset \mathrm{X}$ é estrelado $^{1}$ com respeito à origem $0 \in \mathrm{X}$. Se $d G_{x}$ é simétrica com respeito ao produto interno $\langle\cdot, \cdot\rangle$ para todo $x \in U$, então $G$ é um operador gradiente com respeito à produto interno $\langle\cdot, \cdot\rangle$.

Consideremos $F: U \times V \longrightarrow \mathrm{Z}$, com $U \subset \mathrm{X} \subset \mathrm{Z}, V \subset \mathrm{Y}$, tal que as inclusões são contínuas. Suponha que $F$ atende as condições do teorema 3.1. Além disso, vamos supor que $F$ é um operador gradiente e para todo $y \in V$ a derivada parcial $D_{x} F(\cdot, y)$ é um operador de Fredholm não linear de índice zero. Finalmente, assumimos a decomposição

$$
\mathbf{Z}=\operatorname{Im} D_{x} F\left(x_{0}, y_{0}\right) \oplus \operatorname{Ker} D_{x} F\left(x_{0}, y_{0}\right) .
$$

Esta decomposição define uma projeção

$$
Q: \mathrm{Z} \longrightarrow \mathrm{N}
$$

onde $\mathbf{N}=\operatorname{Ker} D_{x} F\left(x_{0}, y_{0}\right) \cdot Q$ é contínua sobre Z . Pela continuidade da inclusão $\mathbf{X} \subset \mathbf{Z}$, a restrição de $Q$

$$
\left.Q\right|_{\mathrm{X}}: \mathrm{X} \longrightarrow \mathrm{N} \subset \mathrm{X}
$$

também é contínua. Denotaremos por $P=\left.Q\right|_{\mathrm{x}}$. Esta projeção, por sua vez, induz a decomposição

$$
\mathrm{X}=\mathrm{N} \oplus\left(\operatorname{Im} D_{x} F\left(x_{0}, y_{0}\right) \cap \mathbf{X}\right),
$$

onde $\operatorname{Im} D_{x} F\left(x_{0}, y_{0}\right) \cap \mathrm{X}$ é fechado em $\mathrm{X}$.

Usando estas projeções, a redução de Lyapunov-Schmidt do teorema 3.1 tem a seguinte propriedade adicional:

Proposição 3.5. Seja $F: U \times V \longrightarrow Z$ com as propriedades dadas acima. Então a função de dimensão finita $\Phi$ dada pela redução de Lyapunov-Schmidt do teorema (3.1) também é um operador gradiente com respeito à $v$.(O produto interno sobre $\mathbf{Z}$ induz produtos internos sobre $\mathrm{N} \subset \mathrm{X} \subset \mathrm{Z} \mathrm{e}$ estes produtos internos são empregados na definição de um operador gradiente em ambos casos).

\footnotetext{
${ }^{1} \mathrm{O}$ subconjuto $U \subset \mathrm{X}$ é estrelado com respeito à origem $0 \in \mathrm{X}$ se $t x \in U$ para todo $x \in U$ e $t \in[0,1]$.
} 


\subsection{O teorema de bifurcação abstrato de Smoller-Wasserman}

O resultado principal de nosso trabalho é obtido por meio de uma aplicação do teorema de bifurcação sobre domínios variáveis proposto por Lima, Piccione e Zedda em [Lima et al. , 2012, Teo. A.2]. O teorema proposto por Lima, Piccione e Zedda é motivado pelo renomado teorema abstrato de bifurcação apresentado por Smoller e Wasserman em [Smoller e Wasserman , 1990].

Smoller e Wasserman em [Smoller e Wasserman , 1990] consideram uma família de operadores gradiente $\lambda \longrightarrow M_{\lambda}$ de um espaço de Banach fixo $B_{2}$ em outro espaço de Banach fixo $B_{0}$, tais que $B_{2} \subset B_{0}$ continuamente, e uma curva $\lambda \longrightarrow u_{\lambda} \in B_{2}$ que antende à condição $M_{\lambda}\left(u_{\lambda}\right)=0$, para todo $\lambda$. Então o seu resultado principal em [Smoller e Wasserman , 1990] estabelece condições suficientes para que num instante $\lambda^{*}$ exista um ramo de bifurcação, geral ou equivariante, da curva $u_{\lambda}$. Nas seguintes linhas vamos apresentar, de maneira precisa, este resultado.

Sejam $B_{2}, B_{0}$ espaços de Banach e $\mathrm{H}$ um espaço de Hilbert fixado tal que $B_{2} \subset B_{0} \subset \mathrm{H}$, com inclusões contínuas. Seja $\Lambda \subset \mathbb{R}$ um intervalo e $M: \Lambda \times B_{2} \longrightarrow B_{0}$ um operador de classe $C^{1}$. Para cada $\lambda \in \Lambda$, denotamos por $M_{\lambda}$ o operador $M_{\lambda}: B_{2} \longrightarrow B_{0}$, definido por $M_{\lambda}(w)=M(\lambda, w), w \in$ $B_{2}$. Sejam $\left\{u_{\lambda}\right\} \subset B_{2}$ uma família 1-paramétrica contínua, satisfazendo $M\left(\lambda, u_{\lambda}\right)=0$ e $P_{\lambda}$ o subespaço gerado por

$$
\left\{v \in B_{2} \mid\left(d M_{\lambda}\right)_{u_{\lambda}}(v)=\mu v \quad \text { para algum } \mu \geq 0, v \neq 0\right\} .
$$

Teorema 3.2 (Teorema de bifurcação abstrato de Smoller-Wasserman). Seja $M$ o operador definido acima. Suponha que para cada $\lambda \in \Lambda, M_{\lambda}$ é um operador gradiente, tal que $M_{\lambda}(0)=0$. Além disso, assuma que para todo $\lambda \in \Lambda$, existe $\epsilon>0$, tal que

$$
\operatorname{dim} P_{\lambda, \epsilon}=\operatorname{dim}\left\{v \in B_{2} \mid\left(\left(d M_{\lambda}\right)_{0}+\epsilon I\right)(v)=\mu v \text {, para algum } \mu \geq 0, v \neq 0\right\}<\infty .
$$

Se existir $\lambda_{1}<\lambda_{2}$ em $\Lambda$, tais que

i) $\left(d M_{\lambda_{i}}\right)_{0}$ é não singular, $i=1,2$.

ii) $P_{\lambda_{1}}$ não é isomorfo a $P_{\lambda_{2}}$.

Então existe um $\lambda_{0} \in\left(\lambda_{1}, \lambda_{2}\right)$ tal que $\left(\lambda_{0}, 0\right)$ é um instante de bifurcação para a família $M(\lambda, u)=0$.

Como já mencionamos acima, Smoller e Wasserman em [Smoller e Wasserman , 1990] também provam uma versão equivariante do teorma de bifurcação abstrato, mas não precisaremos dela, portanto não discutiremos a dita versão aqui.

\subsection{Teorema de bifurcação sobre domínio variável}

Como já vimos, o teorema de bifurcação abstrato provado por Smoller e Wasserman se aplica para operadores gradiente $M_{\lambda}$ definidos sobre um espaço de Banach fixo $B_{2}$ e tomando valores em outro espaço de Banach fixo $B_{0}$.

Uma configuração um pouco mais geral foi proposta por Lima, Piccione e Zedda em [Lima et al. , 2012]. Basicamente os autores consideram operadores gradiente $F_{\lambda}$ definidos sobre subvariedades de Banach $\mathcal{D}_{\lambda}$ de um espaço de Banach fixo, as quais variam suavemente, e tomando valores numa família de subespaços fechados $\mathcal{E}_{\lambda}$ de um espaço de Banach, que variam suavemente $\operatorname{com} \lambda$. Mais exatamente,

Definição 3.2. Seja $B$ um espaço de Banach. Uma família de subvariedades de Banach de $B$, $[a, b] \ni t \longrightarrow \mathcal{D}_{t} \subset B$, é chamada de família $C^{1}$ de subvariedades de $B$, se o conjunto

$$
\mathcal{D}=\left\{(v, t) \in B \times[a, b] \mid v \in \mathcal{D}_{t}\right\},
$$


tem estrutura de um subfibrado $C^{1}$ do fibrado trivial $B \times[a, b]$. Analogamente, podemos definir uma família $C^{1}$ de subespaços fechados de $B$ como uma família $[a, b] \ni t \longrightarrow \mathcal{E}_{t} \subset B$ de subespaços de Banach de $B$ tal que o conjunto

$$
\mathcal{E}=\left\{(v, t) \in B \times[a, b] \mid v \in \mathcal{E}_{t}\right\},
$$

é um subfibrado do fibrado trivial $B \times[a, b]$.

Por exemplo, se $f: B \times[a, b] \longrightarrow \mathbb{R}$ é uma função de classe $C^{1}$ tal que $D_{x} f(u) \neq 0$ para todo $u \in f^{-1}(0)$, então a família $\mathcal{D}_{\lambda}=\{(u, \lambda) \mid f(u, \lambda)=0\}$ é uma família $C^{1}$ de subvariedades de $B$. Além disso, se $\lambda \longrightarrow x_{\lambda} \in B$ é uma curva $C^{1}$ e $\mathcal{D}=\bigcup_{\lambda}\left(\mathcal{D}_{\lambda} \times\{\lambda\}\right)$ é uma família $C^{1}$ de subvariedades de $B$, com $x_{\lambda} \in \mathcal{D}_{\lambda}$, então a família $\lambda \longrightarrow T_{x_{\lambda}} B_{\lambda}$ é uma família $C^{1}$ de subespaços fechados de $B$.

Teorema 3.3. Dados $B_{0}, B_{2}$ espaços de Banach e $H$ um espaço Hilbertizável. Sejam $[a, b] \ni \lambda \longrightarrow$ $\mathcal{D}_{\lambda} \subset B_{2}$ uma família $C^{1}$ de subvariedades de $B_{2},[a, b] \ni \lambda \longrightarrow \mathcal{E}_{\lambda} \subset B_{0}$ e $[a, b] \ni \lambda \longrightarrow H_{\lambda} \subset H$ famílias $C^{1}$ de subespaços fechados de $B_{0}$ e $H$, respectivamente. Seja $F: \mathcal{D} \longrightarrow \mathcal{E}$ um morfismo de fibrados de classe $C^{1}$ e assuma que as seguintes hipóteses são satisfeitas:

i) $\lambda \longrightarrow e_{\lambda} \in \mathcal{E}_{\lambda}$ é uma seção $C^{1}$ do fibrado $\mathcal{E}$.

ii) $\lambda \longrightarrow d_{\lambda} \in \mathcal{D}_{\lambda}$ é uma seção $C^{1}$ do fibrado $\mathcal{D}$, tal que

$$
F\left(d_{\lambda}, \lambda\right)=\left(e_{\lambda}, \lambda\right) \quad \text { para todo } \lambda \in[a, b]
$$

iii) $\lambda \longrightarrow\langle\cdot, \cdot\rangle_{\lambda}$ é uma família $C^{1}$ de produtos internos completos em $H_{\lambda}$.

iv) Existem inclusões contínuas $B_{2} \subset B_{0} \subset H$ que induz inclusões $T_{d_{\lambda}} \mathcal{D}_{\lambda} \subset \mathcal{E}_{\lambda} \subset H_{\lambda}$ para todo $\lambda \in[a, b]$.

v) Para todo $\lambda$, a função $F_{\lambda}=F(\cdot, \lambda): \mathcal{D}_{\lambda} \longrightarrow \mathcal{E}_{\lambda}$ é um operador gradiente, com respeito à $\langle\cdot, \cdot\rangle_{\lambda}$, em $d_{\lambda}$.

vi) O operador $d F(\cdot, \lambda): T_{d_{\lambda}} \mathcal{D}_{\lambda} \longrightarrow \mathcal{E}_{\lambda}$ é de Fredholm de índice zero para todo $\lambda \in[a, b]$.

vii) Para todo $\lambda \in[a, b]$, existe uma base ortonormal, respeito à $\langle\cdot, \cdot\rangle_{\lambda}, e_{1}^{\lambda}, e_{2}^{\lambda}, \ldots$ de $H_{\lambda}$ consistindo de autovalores de $d F(\cdot, \lambda)$.

viii) Os autovalores correspondentes têm multiplicidade finita e para todo $\lambda \in[a, b]$, o número de autovalores negativos de $d F(\cdot, \lambda)$, denotado por $n_{\lambda}$, é finito.

ix) Existe $\lambda_{*} \in(a, b)$ tal que, para todo $\epsilon>0$ suficientemente pequeno, temos

- $d F\left(\cdot, \lambda_{*}-\epsilon\right)$ e $d F\left(\cdot, \lambda_{*}+\epsilon\right)$ são invertíveis.

- $n_{\lambda_{*}-\epsilon} \neq n_{\lambda_{*}+\epsilon}$.

Então $\lambda_{*}$ é um instante de bifurcação para a equação

$$
F(\cdot, \lambda)=\left(e_{\lambda}, \lambda\right),
$$

isto é, existem sequências $d_{n} \in B_{2}$ e $\lambda_{n} \in[a, b]$, com $d_{n} \in \mathcal{D}_{\lambda_{n}}$ para todo $n$, tais que $\lim _{n \longrightarrow \infty} \lambda_{n}=\lambda_{*}$, $\lim _{n \longrightarrow \infty} d_{n}=d_{\lambda_{*}}, d_{n} \neq d_{\lambda_{n}}$ para todo $n, \mathrm{e}$

$$
F\left(d_{n}, \lambda_{n}\right)=\left(e_{\lambda_{n}}, \lambda_{n}\right) \text { para todo } n \text {. }
$$

Nota 3.1. O critério fornecido pelo teorema acima diz que, sob uma condição local de Palais-Smale, se $d_{1}$ e $d_{2}$ são pontos críticos não degenerados de índice de Morse diferente, então qualquer curva de pontos críticos juntando $d_{1}$ e $d_{2}$ tem pelo menos um instante de bifurcação. 


\section{Capítulo 4}

\section{Fundamento variacional do problema de Yamabe}

O propósito deste capítulo é expor, brevemente, uma configuração variacional para o problema de Yamabe sobre variedades com bordo. As principais referências deste capítulo são [Escobar , 1992b], [Aubin , 1998], [Escobar , 2002], [Agmon et al. , 1959], [Lima et al. , 2012] e [Taylor , 2011a].

Escobar em [Escobar, 1992b], como uma generalização do teorema de uniformização para dimensões superiores, formula a seguinte pergunta:

Seja $(M, g)$ uma variedade Riemanniana compacta, com bordo e $m=\operatorname{dim} M \geq 3$. Quando existe uma métrica conforme $\tilde{g}=\varphi^{\frac{4}{m-2}} g$ de curvatura escalar zero, tal que o bordo $\partial M$ é uma hipersuperficie de curvatura média constante?

No mesmo artigo, [Escobar , 1992b], Escobar dá diferentes condições para garantir a existência de uma tal métrica. Contribuções de diferentes autores, por exemplo [Marques , 2005], [Marques , 2007] e [Almaraz , 2010], têm ampliado o tipo de variedades para as quais a resposta à pergunta feita por Escobar é afirmativa.

Das equações (2.19) e (2.20) segue que o problema de Yamabe proposto pelo Escobar é equivalente à encontrar uma solução positiva ao problema não linear com condições de bordo

$$
\begin{cases}\Delta_{g} \varphi+c_{m} \varphi R_{g}=0 & \text { em } M, \\ \frac{\partial \varphi}{\partial \eta^{g}}+d_{m} \varphi H_{g}=d_{m} H_{\tilde{g}} \varphi^{\frac{m}{m-2}} & \text { sobre } \partial M,\end{cases}
$$

onde $c_{m}=\frac{m-2}{4(m-1)}$ e $d_{m}=\frac{m-2}{2}$. Técnicas variacionais são usadas no estudo do problema não linear (4.1). Por exemplo, dado o espaço de Hilbert $\mathrm{H}^{1}(M)=\mathrm{H}_{2}^{1}(M)$ (definido na subseção 2.2.3) com produto interno

$$
\left\langle\varphi_{1}, \varphi_{2}\right\rangle_{\mathrm{H}^{1}}=\int_{M}\left(g\left(\operatorname{grad}_{g} \varphi_{1}, \operatorname{grad}_{g} \varphi_{2}\right)+\varphi_{1} \varphi_{2}\right) v_{g}
$$

Escobar em [Escobar, 1992b] define o funcional $E: \mathrm{H}^{1}(M) \longrightarrow \mathbb{R}$ por

$$
E(\varphi)=\int_{M}\left(g\left(\operatorname{grad}_{g} \varphi, \operatorname{grad}_{g} \varphi\right)+\frac{m-2}{4(m-1)} R_{g} \varphi^{2}\right) v_{g}+\frac{m-2}{2} \int_{\partial M} H_{g} \varphi^{2} \sigma_{g},
$$

e prova que pontos críticos de $E$ sob certas restrições são soluções positivas de (4.1). Mais exatamente,

Proposição 4.1. Seja $E$ o funcional definido acima. Uma função $\varphi \in \mathrm{H}^{1}(M)$ é um ponto crítico de $E$ sob a restrição

$$
\mathcal{C}=\left\{\varphi \in \mathrm{H}^{1}(M) \mid \int_{\partial M} \varphi^{\frac{2(m-1)}{m-2}} \sigma_{g}=1\right\}
$$

se e só se $\varphi \in C^{\infty}(M), \varphi>0$ e a métrica conforme $\tilde{g}=\varphi^{\frac{4}{m-2}} g$ tem curvatura escalar nula e o bordo $\partial M$ tem curvatura média, com respeito a $\tilde{g}$, constante. 
A proposição 4.1 carateriza as soluções positivas de (4.1), e portanto as métricas conforme à $g$ de curvatura escalar zero e curvatura média do bordo constante, como pontos críticos de $E$ sob a restrição (4.3). Mas para nosso objetivo, precisamos reformular dita caracterização.

Do apêndice B temos que

$$
\mathrm{H}^{1}(M)=\mathrm{H}_{0}^{1}(M) \oplus \mathrm{H}_{\Delta}^{1}(M),
$$

onde

$$
\mathrm{H}_{0}^{1}(M)=\left\{\phi \in \mathrm{H}^{1}(M)|\phi|_{\partial M}=0\right\},
$$

e $\mathrm{H}_{\Delta}^{1}(M)$ denota o subespaço de $\mathrm{H}^{1}(M)$ das funções harmônicas, isto é, $\varphi \in \mathrm{H}_{\Delta}^{1}(M)$ se

$$
\int_{M} g\left(\operatorname{grad}_{g} \varphi, \operatorname{grad}_{g} \phi\right) v_{g}=0, \quad \forall \phi \in \mathrm{H}_{0}^{1}(M)
$$

É claro que $\mathrm{H}_{\Delta}^{1}(M)$ é um subespaço de Hilbert de $\mathrm{H}^{1}(M)$. Como a inclusão $\mathrm{H}^{1}(M) \hookrightarrow \mathrm{L}_{p}(\partial M)$, com $p=\frac{2(m-1)}{m-2}$, é contínua, ver teorema 2.7, podemos definir $\mathcal{H}_{1}(M, g)$ como

$$
\mathcal{H}_{1}(M, g)=\left\{\varphi \in \mathrm{H}_{\Delta}^{1}(M) \mid \int_{\partial M} \varphi^{\frac{2(m-1)}{m-2}} \sigma_{g}=1\right\} \text {. }
$$

Lembre que a classe conforme $[g]$ de $g$ é definida como

$$
[g]=\left\{\tilde{g} \mid \tilde{g}=\varphi^{\frac{4}{m-2}} g, \text { para alguma } \varphi \in C^{\infty}(M), \varphi>0\right\},
$$

$\mathrm{Na}$ seguinte proposição caraterizamos as métricas na classe conforme de $g$ com curvatura escalar zero e curvatura média do bordo constante como pontos crítico do funcional $E$, restrito à $\mathcal{H}_{1}(M, g)$. Além disso, apresentamos uma expressão para a segunda variação de $E$.

Proposição 4.2. Seja $(M, g)$ uma variedade Riemanniana compacta, orientada, com bordo e dimensão $m \geq 3$.

i) $\mathcal{H}_{1}(M, g)$ é uma subvariedade de codimensão 1 de $\mathrm{H}_{\Delta}^{1}(M)$. Além disso, para cada $\varphi \in$ $\mathcal{H}_{1}(M, g)$ o espaço tangente $T_{\varphi} \mathcal{H}_{1}(M, g)$ de $\mathcal{H}_{1}(M, g)$ em $\varphi$ é dado por

$$
T_{\varphi} \mathcal{H}_{1}(M, g)=\left\{\phi \in \mathrm{H}_{\Delta}^{1}(M) \mid \int_{\partial M} \varphi^{\frac{m}{m-2}} \phi \sigma_{g}=0\right\} .
$$

ii) Suponha que a métrica $g$ tenha curvatura escalar nula. Então $\varphi \in \mathcal{H}_{1}(M, g)$ é um ponto crítico de $E$ restrito a $\mathcal{H}_{1}(M, g)$ se e só se $\varphi$ é suave, positiva e a métrica conforme $\tilde{g}=\varphi^{\frac{4}{m-2}} g$ tem curvatura escalar zero e o bordo $\partial M$ tem curvatura média, respeito à $\tilde{g}$, constante.

Além disso, se $\int_{\partial M} \sigma_{g}=1, R_{g}=0$ e $H_{g}$ é constante, isto é, se $\varphi_{0}=\mathbf{1}^{1}$ é um ponto crítico de $E$ restrito a $\mathcal{H}_{1}(M, g)$, então

iii) A segunda variação $d^{2} E_{\varphi_{0}}$ de $E$ em $\varphi_{0}$ é dada por

$$
\left(\phi_{1}, \phi_{2}\right) \longrightarrow 2\left\{\int_{M} g\left(\operatorname{grad}_{g} \phi_{1}, \operatorname{grad}_{g} \phi_{2}\right) v_{g}-H_{g} \int_{\partial M} \phi_{1} \phi_{2} \sigma_{g}\right\}
$$

onde $\phi_{i} \in \mathrm{H}_{\Delta}^{1}(M)$ é tal que $\int_{\partial M} \phi_{i} \sigma_{g}=0, i=1,2$.

iv) Existe um operador (não limitado) autoadjunto $J_{g}: \mathrm{L}_{2}(\partial M) \longrightarrow \mathrm{L}_{2}(\partial M)$, tal que

$$
d^{2} E_{\varphi_{0}}\left(\phi_{1}, \phi_{2}\right)=2 \int_{\partial M} J_{g}\left(\left.\phi_{1}\right|_{\partial M}\right) \phi_{2} \sigma_{g}
$$

$\phi_{i} \in \mathrm{H}_{\Delta}^{1}(M)$ tal que $\int_{\partial M} \phi_{i} \sigma_{g}=0, i=1,2$.

\footnotetext{
${ }^{1}$ Aqui 1 denota a função constante 1 sobre $M$
} 
Demonstração. Seja $\mathcal{V}_{g}: \mathrm{H}_{\Delta}^{1}(M) \longrightarrow \mathbb{R}$ definida por

$$
\mathcal{V}_{g}(\varphi)=\int_{\partial M} \varphi^{\frac{2(m-1)}{m-2}} \sigma_{g}
$$

Claramente $\mathcal{V}_{g}$ é suave e sua diferencial em $\varphi \in \mathrm{H}_{\Delta}^{1}(M)$ é dada por

$$
\left(d \mathcal{V}_{g}\right)_{\varphi}(\phi)=\frac{2(m-1)}{m-2} \int_{\partial M} \varphi^{\frac{m}{m-2}} \phi \sigma_{g}, \quad \text { para toda } \phi \in \mathrm{H}_{\Delta}^{1}(M)
$$

Note que $\mathcal{H}_{1}(M, g)=\mathcal{V}_{g}^{-1}(1)$. Logo, para estabelecer (i) é suficiente mostrar que $\mathcal{V}_{g}$ restrita a $\mathcal{H}_{1}(M, g)$ é uma submersão. Com efeito, dada $\varphi \in \mathcal{H}_{1}(M, g)$ para $\phi=\varphi$ obtemos

$$
\left(d \mathcal{V}_{g}\right)_{\varphi}(\phi)=\frac{2(m-1)}{m-2} \int_{\partial M} \varphi^{\frac{2(m-1)}{m-2}} \sigma_{g}=\frac{2(m-1)}{m-2}>0,
$$

portanto $\left(d \mathcal{V}_{g}\right)_{\varphi}$ é sobrejetora para toda $\varphi \in \mathcal{H}_{1}(M, g)$. Obviamente $\operatorname{Ker}\left(d \mathcal{V}_{g}\right)_{\varphi}$ é complementado em $\mathrm{H}_{\Delta}^{1}(M)$ para todo $\varphi \in \mathcal{H}_{1}(M, g)$. Assim, $\mathcal{V}_{g}$ restrita a $\mathcal{H}_{1}(M, g)$ é uma submersão. (4.6) segue do fato que

$$
\operatorname{Ker}\left(d \mathcal{V}_{g}\right)_{\varphi}=\left\{\phi \in \mathrm{H}_{\Delta}^{1}(M) \mid \int_{\partial M} \varphi^{\frac{m}{m-2}} \phi \sigma_{g}=0\right\} .
$$

Por outro lado, supondo $R_{g}=0, \varphi$ é um ponto crítico de $E$ restrito $\mathcal{H}_{1}(M, g)$ se e só se

$$
d^{2} E_{\varphi_{0}}(\phi) \int_{M} g\left(\operatorname{grad}_{g} \varphi, \operatorname{grad}_{g} \phi\right) v_{g}+\frac{m-2}{2} \int_{\partial M} H_{g} \varphi \phi \sigma_{g}=0,
$$

para toda $\phi \in \mathrm{H}_{\Delta}^{1}(M)$ tal que

$$
\int_{\partial M} \varphi^{\frac{m}{m-2}} \phi \sigma_{g}=0
$$

Note que (4.10) e (4.11) são validas para toda $\psi \in \mathrm{H}_{0}^{1}(M)$. Logo de (4.4) e (4.10) temos que $\varphi$ é ponto crítico de $E$ restrito a $\mathcal{H}_{1}(M, g)$ se e só se

$$
d E_{\varphi}(\phi)=\int_{M} g\left(\operatorname{grad}_{g} \varphi, \operatorname{grad}_{g} \phi\right) v_{g}+\frac{m-2}{2} \int_{\partial M} H_{g} \varphi \phi \sigma_{g}=0
$$

para toda $\phi \in \mathrm{H}^{1}(M)$ tal que

$$
\int_{\partial M} \varphi^{\frac{m}{m-2}} \phi \sigma_{g}=0
$$

Portanto (ii) segue da proposição 4.1.

Assuma $\varphi_{0}=\mathbf{1}$ é um ponto crítico de $E$ restrito à $\mathcal{H}_{1}(M, g)$. Pelo método dos multiplicadores de Lagrange existe um escalar $\lambda$ tal que

$$
d E_{\varphi_{0}}(\phi)-\lambda\left(d \mathcal{V}_{g}\right)_{\varphi_{0}}(\phi)=0,
$$

para toda $\phi \in \mathrm{H}^{1}(M)$. Logo de (4.10) e (4.9) temos

$$
\int_{\partial M}\left(\frac{m-2}{2} H_{g}-\frac{(m-1) \lambda}{m-2}\right) \phi \sigma_{g}=0,
$$

para toda $\phi \in \mathrm{H}^{1}(M)$. Assim, pelo lema fundamental do cálculo das variações

$$
\frac{(m-1) \lambda}{m-2}=\frac{m-2}{2} H_{g} .
$$


Dada $\phi \in \mathrm{H}_{\Delta}^{1}(M), \operatorname{com} \int_{\partial M} \phi \sigma_{g}=0$, seja $E(\phi): \mathcal{H}_{1}(M, g) \longrightarrow \mathbb{R}$ definido por

$$
E(\phi)(\varphi)=d E_{\varphi}(\phi)-\lambda\left(d \mathcal{V}_{g}\right)_{\varphi}(\phi)
$$

Então a segunda variação $d^{2} E_{\varphi_{0}}$ de $E$ em $\varphi_{0}$ restrito à $\mathcal{H}_{1}(M, g)$ é dada por

$$
\begin{aligned}
d^{2} E_{\varphi_{0}}\left(\phi_{1}, \phi_{2}\right) & =\left(d E\left(\phi_{1}\right)_{\varphi_{0}}\right)\left(\phi_{2}\right), \\
& =2\left\{\int_{M} g\left(\operatorname{grad}_{g} \phi_{1}, \operatorname{grad}_{g} \phi_{2}\right) v_{g}+\frac{m-2}{2} \int_{\partial M} H_{g} \phi_{1} \phi_{2} \sigma_{g}-\frac{m(m-1) \lambda}{(m-2)^{2}} \int_{\partial M} \phi_{1} \phi_{2} \sigma_{g}\right\}, \\
& =2\left\{\int_{M} g\left(\operatorname{grad}_{g} \phi_{1}, \operatorname{grad}_{g} \phi_{2}\right) v_{g}-H_{g} \int_{\partial M} \phi_{1} \phi_{2} \sigma_{g}\right\},
\end{aligned}
$$

para toda $\phi_{1}, \phi_{2} \in \mathrm{H}_{\Delta}^{1}(M)$ tais que $\int_{\partial M} \phi_{1} \sigma_{g}=0=\int_{\partial M} \phi_{2} \sigma_{g}$. Na terceira igualdade usamos o fato que $\frac{(m-1) \lambda}{m-2}=\frac{m-2}{2} H_{g}$.

Finalmente, da proposição B.2 no apêndice B temos

$$
\int_{\partial M} g\left(\operatorname{grad}_{g} \phi_{1}, \operatorname{grad}_{g} \phi_{2}\right) v_{g}=\int_{\partial M} \mathcal{N}_{g}\left(\left.\phi_{1}\right|_{\partial M}\right) \phi_{2} \sigma_{g} .
$$

onde $\mathcal{N}_{g}$ denota o operador Direchlet-Neumann, ver apêndice B. Logo, de (4.7) temos

$$
\begin{aligned}
d^{2} E_{\varphi_{0}}\left(\phi_{1}, \phi_{2}\right) & =\int_{\partial M} \mathcal{N}_{g}\left(\left.\phi_{1}\right|_{\partial M}\right) \phi_{2} \sigma_{g}-H_{g} \int_{\partial M} \phi_{1} \phi_{2} \sigma_{g} \\
& =\int_{\partial M}\left(\mathcal{N}_{g}\left(\left.\phi_{1}\right|_{\partial M}\right)-H_{g} \phi_{1}\right) \phi_{2} \sigma_{g} \\
& =\int_{\partial M} J_{g}\left(\left.\phi_{1}\right|_{\partial M}\right) \phi_{2} \sigma_{g}
\end{aligned}
$$

onde $\phi_{1}, \phi_{2} \in T_{\varphi_{0}} \mathcal{H}_{1}(M, g)$ e $J_{g}=\mathcal{N}_{g}-H_{g}$.

\subsection{O operador de Jacobi}

Assuma que $\varphi_{0}$ é um ponto crítico de $E$ restrito a $\mathcal{H}_{1}(M, g)$. Então, da proposição 4.2 (iv) temos

$$
d^{2} E_{\varphi_{0}}\left(\phi_{1}, \phi_{2}\right)=\int_{\partial M} J_{g}\left(\left.\phi_{1}\right|_{\partial M}\right) \phi_{2} \sigma_{g}, \quad \phi_{1}, \phi_{2} \in T_{\varphi_{0}} \mathcal{H}_{1}(M, g) .
$$

O operador $J_{g}=\mathcal{N}_{g}-H_{g}$ é chamado operador de Jacobi. O operador (não limitado)

$$
J_{g}: \mathrm{L}_{2}(\partial M) \longrightarrow \mathrm{L}_{2}(\partial M)
$$

é autoadjunto. Além disso, como a inclusão $T_{\varphi_{0}} \mathcal{H}_{1}(M, g) \hookrightarrow \mathrm{L}_{2}(\partial M)$ é compacta e $\mathcal{N}_{g}$ é um operador de Fredholm de índice zero, ver proposição B.3, então $J_{g}$ é um operador de Fredholm de índice zero. Ainda mais, como no apêndice $\mathrm{B}$, o espaço $T_{\varphi_{0}} \mathcal{H}_{1}(M, g)$ pode ser identificado com o subespaço $\mathrm{V}_{0}$ de $\mathrm{L}_{2}(\partial M)$ dado por

$$
\mathrm{V}_{0}=\left\{f \in \mathrm{L}_{2}(\partial M) \mid \exists \phi \in T_{\varphi_{0}} \mathcal{H}_{1}(M, g) \quad \text { tal que }\left.\quad \phi\right|_{\partial M}=f\right\} .
$$

Logo, pode-se mostrar, ver [Taylor , 2011b, pag. 44], que

$$
J_{g}\left(\left.\phi\right|_{\partial M}\right) \in T_{\varphi_{0}} \mathcal{H}_{1}(M, g), \quad \phi \in T_{\varphi_{0}} \mathcal{H}_{1}(M, g) .
$$

Portanto, se $J_{g}: T_{\varphi_{0}} \mathcal{H}_{1}(M, g) \longrightarrow T_{\varphi_{0}} \mathcal{H}_{1}(M, g)$ é injetor segue que $J_{g}$ é um isomorfismo.

Note que o espectro do operador de Jacobi $J_{g}$ coincide com o espectro da função Direchlet- 
Neumann deslocado por $H_{g}$. É conhecido que o espectro de $\mathcal{N}_{g}$ coincide com os autovalores de Steklov, isto é, com as soluções do sistema

$$
\begin{cases}\Delta_{g} \psi=0 & \text { sobre } \quad M \\ \psi=\rho \psi & \text { em } \quad \partial M\end{cases}
$$

Portanto os autovalores de $J_{g}$ são dados por

$$
\rho_{1}-H_{g} \leq \rho_{2}-H_{g} \leq \rho_{3}-H_{g} \leq \ldots,
$$

onde $\rho_{j}$ denota o $j$-ésimo autovalor de Steklov, repetido de acordo a sua multiplicidade.

Nota 4.1. Observe que as autofuncões de $J_{g}$ coincidem com as autofuncões de $\mathcal{N}_{g}$. Além disso, $-H_{g}$ não é um autovalor de $J_{g}$ pois o seu domínio não contem as funcões constantes.

Proposição 4.3. Assuma que $\varphi_{0}$ é um ponto crítico de $E$ restrito a $\mathcal{H}_{1}(M, g)$. Então

i) O índice de Morse de $\varphi_{0}$ é finito. Alem disso, o índice de Morse de $\varphi_{0}$ é dado por

$$
\max \left\{j \in \mathbb{N} \mid \rho_{j}<H_{g}\right\} .
$$

ii) $\operatorname{Ker}\left(d^{2} E_{\varphi_{0}}\right)=\operatorname{Ker}\left(J_{g}\right)$, e a nulidade de $\varphi_{0}$, denotada por $\nu(g)$, é dada por

$$
\nu(g)=\operatorname{dim} \operatorname{Ker}\left(J_{g}\right)
$$

Demonstração. Ver proposição 5 em [Ramírez, 2014].

Nota 4.2. Observe que se $H_{g} \leq 0$, então o kernel de $J_{g}$ é trivial. 


\section{Capítulo 5}

\section{Bifurcação no problema de Yamabe}

O objetivo principal neste capítulo é provar um críterio para garantir a existência de instantes de bifurcação para uma família de métricas, sobre uma variedade compacta com bordo $M$, de curvatura escalar nula e tal que o bordo é uma subvariedade de curvatura média constante. Iniciamos o capítulo expondo a estrutura do conjunto das métricas Riemannianas e continuamos definindo instante de bifurcação no contexto do problema de Yamabe de nosso interesse. Na seção 5.1 mostramos um resultado sobre a convergência num ramo de bifurcação. Finalmente, na seção 5.2 damos um críterio para a existência de instantes de bifurcação. As principais referências neste capítulo são [Clarke , 2010], [Agmon et al. , 1959], [Lions e Magenes , 1972], [Taylor , 2011a,b], [Aubin , 1998], [Lima et al. , 2012] e [Escobar, 2002].

Neste capítulo $M$ denotará uma variedade Riemanniana compacta orientada de dimensão $m \geq 3$ com bordo. Dada uma métrica Riemanniana $g_{0}$ sobre M, tal como vimos na seção 2.1 a métrica $g_{0}$ induz um produto interno e normas em todos os fibrados tensoriais sobre $M$ e a conexão Riemanniana $\nabla^{g_{0}}$ associada a $g_{0}$ induz uma conexão em todos os espaços dos campos tensoriais sobre $M$. Seja $\mathcal{S}^{k}, k \geq 2$, o espaço dos (2,0)-campos tensoriais simétricos de classe $C^{k}$ sobre $M$, este espaço é um espaço de Banach com a norma

$$
\|\tau\|_{C^{k}}=\max _{j=0, \ldots, k}\left[\max _{p \in M}\left\|\nabla^{(j)} \tau(p)\right\|\right]
$$

O conjunto $\operatorname{Met}^{k}(M)$ de todas as métricas Riemannianas de classe $C^{k}$ sobre $M$ é um subconjunto aberto de $\mathcal{S}^{k}$, e portanto uma variedade de Banach. O espaço tangente $T_{g} \operatorname{Met}^{k}(M)$ de $\operatorname{Met}^{k}(M)$ em $g$ é identificado com o espaço $\mathcal{S}^{k}$, para toda $g \in \operatorname{Met}^{k}(M)$.

Denotaremos por $\mathcal{M}$ o subconjunto de $\operatorname{Met}^{k}(M)$ dado por

$$
\mathcal{M}=\left\{g \in \operatorname{Met}^{k}(M) \mid \int_{\partial M} \sigma_{g}=1\right\} .
$$

Proposição 5.1. $\mathcal{M}$ é uma subvariedade (de Banach) de $\operatorname{Met}^{k}(M)$.

Demonstração. Seja $\mathcal{V}: \operatorname{Met}^{k}(M) \longrightarrow \mathbb{R}$ definida por

$$
\mathcal{V}(g)=\int_{\partial M} \sigma_{g}
$$

onde $\sigma_{g}$ denota a forma de volume sobre $\partial M$ induzida por $g$. Dado que $\mathcal{M}=\mathcal{V}^{-1}(1)$ o resultado segue de provar que $\mathcal{V}$ é uma submersão, ver proposição 2.12. Note que $\mathcal{V}$ é suave e sua diferencial é dada por

$$
d \mathcal{V}_{g}(h)=\frac{1}{2} \int_{\partial M} \operatorname{tr}_{g}(h) \sigma_{g}=\frac{1}{2} \int_{\partial M}\langle h, g\rangle_{g} \sigma_{g}, \quad \text { para todo } h \in \mathcal{S}^{k}
$$

Aqui $t r_{g}$ denota o traço de $\left.h\right|_{\partial M}$ com respeito à $\left.g\right|_{\partial M}$ e $\langle\cdot, \cdot\rangle_{g}$ denota o produto interno induzido por 
$\left.g\right|_{\partial M}$ sobre todos os espaços dos campos tensoriais definidos sobre $\partial M$. Para $h=g$, de (5.2) temos

$$
d \mathcal{V}_{g}(h)=\frac{m-1}{2} \int_{\partial M} \sigma_{g}=\frac{(m-1)}{2} \operatorname{Vol}_{g}(\partial M)>0 .
$$

Portanto $d \mathcal{V}_{g}$ é sobrejetora para toda $g \in \operatorname{Met}^{k}(M)$. De (5.2) segue que o Ker $d \mathcal{V}_{g}$ é o subespaço de $\mathcal{S}^{k}$ dos campos tensoriais $h$ tais que $\int_{\partial M} \operatorname{tr}_{g}(h) \sigma_{g}=0$. Dado $h \in \mathcal{S}^{k}$, denotamos por $\lambda_{h}=$ $\frac{\int_{\partial M} \operatorname{tr}_{g}(h) \sigma_{g}}{(m-1) \operatorname{Vol}_{g}(\partial M)}$. Então

$$
\tilde{h}=h-\lambda_{h} g \in \operatorname{Ker} d \mathcal{V}_{g}
$$

Assim,

$$
h=\lambda_{h} g+\tilde{h}, \quad \operatorname{com} \quad \tilde{h} \in \operatorname{Ker} d \mathcal{V}_{g} .
$$

Logo Ker $d \mathcal{V}_{g}$ é complementado em $\mathcal{S}^{k}$, para toda $g \in \operatorname{Met}^{k}(M)$. Portanto $\mathcal{V}$ é uma submersão.

Seja $[a, b] \ni t \longrightarrow g_{t} \in \mathcal{M}$ uma curva suave tal que $g_{t}$ tem curvatura escalar nula e a curvatura média $H_{g_{t}}$ do bordo é constante, para todo $t \in[a, b]$. Vamos definir o que é um instante de bifurcação para a família de métricas $\left(g_{t}\right)_{t \in[a, b]}$.

Definição 5.1. Seja $[a, b] \ni t \longrightarrow g_{t} \in \mathcal{M}$ uma curva suave como acima. Um elemento $t_{*} \in[a, b]$ é chamado um instante de bifurcação para a família $\left(g_{t}\right)_{t \in[a, b]}$ se existem duas sequências $\left(t_{n}\right) \subset[a, b]$ e $\left(\varphi_{n}\right) \subset \mathrm{H}^{1}(M)$ tais que

a) $\varphi_{n}$ é um ponto crítico de $E$ restrito a $\mathcal{H}_{1}\left(M, g_{t_{n}}\right)$ para todo $n$.

b) $\varphi_{n} \neq \mathbf{1}$ para todo $n$.

c) $t_{n} \longrightarrow t_{*}$ quando $n \longrightarrow \infty$.

d) $\varphi_{n} \longrightarrow \mathbf{1}$ quando $n \longrightarrow \infty \mathrm{em} \mathrm{H}^{1}(M)$.

Se $t_{*} \in[a, b]$ não é um instante de bifurcação, então diremos que a família $\left(g_{t}\right)_{t \in[a, b]}$ é localmente rígida em $t_{*}$.

Note que a existência de um instante de bifurcação $t_{*}$ para a família de métricas $\left(g_{t}\right)_{t \in[a, b]}$ garante a existência de um número infinito de classes conformes, numa vizinhança da classe conforme $\left[g_{t_{*}}\right]$, com pelo menos duas métricas não homotéticas de curvatura escalar nula e curvatura média do bordo constante.

\subsection{Convergência de um ramo de bifurcação}

Seja $[a, b] \ni t \longrightarrow g_{t} \in \mathcal{M}$ uma curva suave tal que $g_{t}$ tem curvatura escalar nula e o bordo $\partial M$ tem curvatura média $H_{g_{t}}$ constante, para todo $t \in[a, b]$. Suponha que $t_{*} \in[a, b]$ é um instante de bifurcação para a família $\left(g_{t}\right)_{t \in[a, b]}$. Nesta seção vamos provar que a família de métricas conforme $\left(g_{n}\right)$ dadas por

$$
g_{n}=\varphi_{n}^{\frac{4}{m-2}} g_{t_{n}}
$$

onde $\left(t_{n}\right) \subset[a, b]$ e $\left(\varphi_{n}\right) \subset \mathrm{H}^{1}(M)$ são as sequências dadas na definição 5.1, converge a $g_{t_{*}}$ em $\mathcal{M}$ sempre que $m \geq 4$.

Proposição 5.2. Seja $[a, b] \ni t \longrightarrow g_{t} \in \mathcal{M}$ uma curva suave tal que $g_{t}$ tem curvatura escalar nula e o bordo $\partial M$ tem curvatura média constante $H_{g_{t}}$, para todo $t \in[a, b]$. Suponha que $t_{*} \in[a, b]$ é um instante de bifurcação para a família $\left(g_{t}\right)_{t \in[a, b]}$. Seja $\left(g_{n}\right)$ a família de métricas definidas em (5.3) e denote por $H_{n}$ a curvatura média do bordo $\partial M$ com respeito a $g_{n}$. Então

i) $H_{n} \longrightarrow H_{g_{*}}$ quando $n \longrightarrow \infty$. 
ii) Se $m \geq 4$, então $\varphi_{n} \longrightarrow \mathbf{1}$ em $\mathbf{H}_{p}^{s}(M)$, para todo inteiro positivo $s$ e $p=\frac{2(m-2)}{m}$.

iii) Se $m \geq 4$, então $\varphi_{n} \longrightarrow \mathbf{1}$ em $C^{s}(\bar{M})$, para todo inteiro positivo $s$.

Demonstração. Da proposição 4.2 temos que a métrica $g_{n}=\varphi_{n}^{\frac{4}{m-2}} g_{t_{n}}$ tem curvatura escalar nula e curvatura média $H_{n}$ do bordo constante para todo $n$. Logo as $\varphi_{n}$ satisfazem o sistema

$$
\begin{cases}\Delta_{g_{t_{n}}} \varphi_{n}=0 & \text { em } M, \\ \frac{\partial \varphi_{n}}{\partial \eta^{t_{n}}}+\frac{m-2}{2} H_{g_{t_{n}}} \varphi_{n}=\frac{m-2}{2} H_{n} \varphi_{n}^{\frac{m}{m-2}} & \text { sobre } \partial M,\end{cases}
$$

onde $\eta^{t_{n}}=\eta^{g_{t_{n}}}$. Da integração por partes em (5.4) temos

$$
H_{n}=\frac{2}{m-2} \int_{M} g_{t_{n}}\left(\operatorname{grad}_{g_{t n}} \varphi_{n}, \operatorname{grad}_{g_{t n}} \varphi_{n}\right) v_{g_{t_{n}}}+H_{g_{t_{n}}} \int_{\partial M} \varphi_{n}^{2} \sigma_{g_{t_{n}}} .
$$

Como $g_{t_{n}} \longrightarrow g_{t_{*}}$ na $C^{k}$-topologia, então

$$
H_{g_{t_{n}}} \longrightarrow H_{g_{t_{*}}}
$$

Afirmamos que

$$
\int_{M} g_{t_{n}}\left(\operatorname{grad}_{g_{t n}} \varphi_{n}, \operatorname{grad}_{g_{t n}} \varphi_{n}\right) v_{g_{t_{n}}} \longrightarrow 0 \quad \text { quando } n \longrightarrow \infty
$$

Com efeito, note que

$$
v_{g_{t_{n}}}=\psi_{n} v_{g_{t_{*}}} \quad \text { e } \sigma_{g_{t_{n}}}=\hat{\psi}_{n} \sigma_{g_{t_{*}}}
$$

onde $\psi_{n}$ e $\hat{\psi}_{n}$ são dadas numa carta por

$$
\psi_{n}=\sqrt{\frac{\left|g_{t_{n}}\right|}{\left|g_{t_{*}}\right|}} \text { e } \quad \hat{\psi}_{n}=\sqrt{\frac{\left|\hat{g}_{t_{n}}\right|}{\left|\hat{g}_{t_{*}}\right|}}
$$

Aqui $\hat{g}_{t_{n}}$ e $\hat{g}_{t_{*}}$ denotam a restrição de $g_{t_{n}}$ e $g_{t_{*}}$ ao bordo $\partial M$, respectivamente. Como $g_{t_{n}} \longrightarrow g_{t_{*}}$ na $C^{k}$-topologia, então $\psi_{n} \longrightarrow \mathbf{1}$ e $\hat{\psi}_{n} \longrightarrow \mathbf{1}$ na $C^{k}$-topologia. Logo, numa carta $(U, \Theta)$ de $M$ temos

$$
\int_{U} g_{t_{n}}\left(\operatorname{grad}_{g_{t n}} \varphi_{n}, \operatorname{grad}_{g_{t n}} \varphi_{n}\right) v_{g_{t_{n}}}=\int_{U} \psi_{n} g_{t_{n}}^{i j} \frac{\partial \varphi_{n}}{\partial x^{i}} \frac{\partial \varphi_{n}}{\partial x^{j}} v_{t_{t_{*}}} .
$$

Note que $\psi_{n} g_{t_{n}}^{i j}$ é uniformemente limitada, logo de (5.8) temos

$$
\left|\int_{U} g_{t_{n}}\left(\operatorname{grad}_{g_{t n}} \varphi_{n}, \operatorname{grad}_{g_{t n}} \varphi_{n}\right) v_{g_{t_{n}}}\right| \leq C \sum_{i, j=1}^{m} \int_{U}\left|\frac{\partial \varphi_{n}}{\partial x^{i}} \frac{\partial \varphi_{n}}{\partial x^{j}}\right| v_{g_{t_{*}}},
$$

para alguma constante $C$. Como $\varphi_{n} \longrightarrow \mathbf{1} \mathrm{em} \mathrm{H}^{1}(M)$ então

$$
\int_{U}\left(\frac{\partial \varphi_{n}}{\partial x^{i}}\right)^{2} v_{g_{t_{*}}} \longrightarrow 0 \quad \text { quando } \quad n \longrightarrow \infty
$$

Assim, de (5.9) e (5.10) temos

$$
\int_{U} g_{t_{n}}\left(\operatorname{grad}_{g_{t n}} \varphi_{n}, \operatorname{grad}_{g_{t n}} \varphi_{n}\right) v_{g_{t_{n}}} \longrightarrow 0 \quad \text { quando } n \longrightarrow \infty .
$$

Como $M$ pode ser coberta por um número finito de cartas, então (5.7) segue de (5.11). Por outro 
lado, a continuidade da inclusão $\mathrm{H}^{1}(M) \subset \mathrm{L}_{2}(\partial M)$ implica que

$$
\int_{\partial M} \varphi_{n}^{2} \sigma_{g_{t_{n}}}=\int_{\partial M} \hat{\psi}_{n} \varphi_{n}^{2} \sigma_{g_{t_{*}}} \longrightarrow 1 \quad \text { quando } n \longrightarrow+\infty \text {. }
$$

Portanto de (5.5), (5.6), (5.7) e (5.12) temos

$$
H_{n} \longrightarrow H_{g_{*}}, \quad \text { quando } n \longrightarrow \infty \text {. }
$$

A fim de provar (ii) note que $v_{n}=\varphi_{n}-\mathbf{1}$ satisfaz

$$
\begin{cases}\Delta_{g_{t_{n}}} v_{n}=0 & \text { em } M, \\ \frac{\partial v_{n}}{\partial \eta^{t_{n}}}+\frac{m-2}{2} H_{g_{t_{n}}} \varphi_{n}=\frac{m-2}{2} H_{n} \varphi_{n}^{\frac{m}{m-2}} & \text { sobre } \partial M,\end{cases}
$$

para todo $n$. De estimativas elípticas padrão, ver [Agmon et al. , 1959, Teor.15.2], temos

$$
\begin{aligned}
\left\|\varphi_{n}-\mathbf{1}\right\|_{\mathrm{H}_{p}^{s}(M)} & \leq \\
& C_{n}\left\{\left\|-\frac{m-2}{2} H_{g_{t_{n}}} \varphi_{n}+\frac{m-2}{2} H_{g_{n}} \varphi_{n}^{\frac{m}{m-2}}\right\|_{\mathrm{H}_{p}^{s-1-\frac{1}{p}}(\partial M)}+\left\|\varphi_{n}-\mathbf{1}\right\|_{\mathrm{L}_{p}(M)}\right\},
\end{aligned}
$$

onde $s$ é um inteiro positivo, $1 \leq p=\frac{2(m-1)}{m}<2$ e a constante $C_{n}$ depende só de $s$, a variedade $M$, a $\mathrm{L}_{\infty}$-norma dos coeficientes do operador elíptico $\Delta_{g_{t_{n}}}$, a constante de elipticidade de $\Delta_{g_{t_{n}}}$, e o módulo de continuidade dos coeficientes de $\Delta_{g_{t_{n}}}$. Como $g_{t_{n}}$ converge a $g_{t_{*}}$ na $C^{k}$-topologia então os coeficientes de $\Delta_{g_{t_{n}}}$ convergem uniformemente aos coeficientes de $\Delta_{g_{t_{*}}}$. Assim, em (5.14) pode se escolher uma constante $C n \equiv C$ que não depende de $n$. Do teorema do traço temos que a inclusão de $\mathrm{H}_{p}^{s}(M)$ em $\mathrm{H}_{p}^{s-\frac{1}{p}}(\partial M)$ é contínua, logo de (5.14) segue

$$
\left\|\varphi_{n}-\mathbf{1}\right\|_{\mathrm{H}_{p}^{s}(M)} \leq \bar{C}\left\{\left\|-\frac{m-2}{2} H_{g_{t_{n}}} \varphi_{n}+\frac{m-2}{2} H_{g_{n}} \varphi_{n}^{\frac{m}{m-2}}\right\|_{\mathrm{H}_{p}^{s-1}(M)}+\left\|\varphi_{n}-\mathbf{1}\right\|_{\mathrm{L}_{p}(M)}\right\},
$$

para alguma constante positiva $\bar{C}$. Como a inclusão de $\mathrm{H}^{1}(M)$ em $\mathrm{H}_{p}^{1}(M)$ é contínua, então

$$
\left\|\varphi_{n}-\mathbf{1}\right\|_{\mathrm{L}_{p}(M)} \longrightarrow 0 .
$$

Além disso, devido a que $1 \leq p=\frac{2(m-2)}{m}<2$ a inclusão de $\mathrm{H}^{1}(M)$ em $\mathrm{H}_{p}^{1}(M)$ é contínua. Logo, para $s=2$ temos

$$
\varphi_{n} \longrightarrow \mathbf{1} \text { em } \mathrm{H}_{p}^{s-1}(M) .
$$

Afirmamos que

$$
\varphi_{n}^{\frac{m}{m-2}} \longrightarrow \mathbf{1} \quad \text { em } \quad \mathrm{H}_{p}^{s-1}(M)
$$

Com efeito,

$$
\begin{aligned}
\int_{M}\left(\varphi_{n}^{\frac{m}{m-2}}-1\right)^{\frac{2(m-2)}{m}} v_{g_{*}} & \leq \operatorname{Vol}_{g_{*}}(M)^{\frac{2}{m}}\left(\int_{M}\left(\varphi_{n}^{\frac{m}{m-2}}-1\right)^{2} v_{g_{t_{*}}}\right)^{\frac{m-2}{m}} \\
& =\operatorname{Vol}_{g_{t_{*}}}(M)^{\frac{2}{m}}\left(\int_{M}\left[\left(\varphi_{n}^{\frac{2 m}{m-2}}-1\right)-2\left(\varphi_{n}^{\frac{m}{m-2}}-1\right)\right] v_{g_{t_{*}}}\right)^{\frac{m-2}{2}} .
\end{aligned}
$$

De (5.19) e a continuidade das inclusões de $\mathrm{H}^{1}(M)$ em $\mathrm{L}_{\frac{2 m}{m-2}}(M)$ e $\mathrm{L}_{\frac{m}{m-2}}(M)$, respectivamente, temos

$$
\int_{M}\left(\varphi_{n}^{\frac{m}{m-2}}-1\right)^{\frac{2(m-2)}{m}} v_{g_{*}} \longrightarrow 0 .
$$


Além disso,

$$
\begin{aligned}
\int_{M}\left(\partial_{i} \varphi_{n}^{\frac{m}{m-2}}\right)^{\frac{2(m-2)}{m}} v_{g_{t_{*}}} & =\left(\frac{m}{m-2}\right)^{\frac{m-2}{m}} \int_{M} \varphi_{n}^{\frac{4}{m}}\left(\partial_{i} \varphi_{n}\right)^{\frac{2(m-2)}{m}} v_{g_{t_{*}}} \\
& \leq\left(\frac{m}{m-2}\right)^{\frac{m-2}{m}}\left(\int_{M}\left(\partial_{i} \varphi_{n}\right)^{2} v_{g_{*}}\right)^{\frac{m-2}{m}}\left(\int_{M} \varphi_{n}^{2} v_{g_{t_{*}}}\right)^{\frac{2}{m}}
\end{aligned}
$$

para cada $i, 1 \leq i \leq m$. Como $\varphi_{n} \longrightarrow \mathbf{1} \mathrm{em} \mathrm{H}^{1}(M)$, então o lado direito na desigualdade acima converge a zero. Logo

$$
\int_{M}\left(\partial_{i} \varphi_{n}^{\frac{m}{m-2}}\right)^{\frac{2(m-2)}{m}} v_{g_{*}} \longrightarrow 0
$$

para cada $1 \leq i \leq m$. Nossa afirmação segue de (5.20) e (5.21). Note que para $s=2$, de (5.15), (5.16), (5.17) e (5.18) temos que

$$
\varphi_{n} \longrightarrow \mathbf{1} \quad \text { em } \quad \mathrm{H}_{p}^{s}(M)
$$

$p=\frac{2(m-2)}{m}$. Procedendo indutivamente sobre $s$ na equação (5.15) obtemos

$$
\varphi_{n} \longrightarrow \mathbf{1} \quad \text { em } \mathbf{H}_{p}^{s}(M)
$$

para todo inteiro positivo $s$, isto prova (ii).

Finalmente, (iii) é uma consequência direta da continuidade da inclusão de $\mathrm{H}_{p}^{r+1}(M)$ em $C^{s}(\bar{M})$, a qual é verdade para todo $r>s-1+\frac{m}{p}$, ver teorema 2.7 .

Como consequência da proposição acima temos

Corolario 5.1. Seja $[a, b] \ni t \longrightarrow g_{t} \in \mathcal{M}$ uma curva suave tal que para todo $t \in[a, b], g_{t}$ tem curvatura escalar nula e o bordo $\partial M$ tem curvatura média, com respeito a $g_{t}$, constante. Se $t_{*}$ é um instante de bifurcação para a família $\left(g_{t}\right)_{t \in[a, b]}$, então a família de métricas conforme $\left(g_{n}\right)$, onde $g_{n}$ é definida em (5.3), é tal que $g_{n} \longrightarrow g_{t_{*}}$ em $\mathcal{M}$.

\subsection{Condição suficiente para existência de instantes de bifurcação no problema de Yamabe}

Nesta seção vamos provar uma condição suficiente para garantir a existência de instantes de bifurcação para uma família $\left(g_{t}\right)_{t \in[a, b]}$ de métricas Riemannianas sobre $M$ tal que a curvatura escalar de $g_{t}$ é nula e o bordo $\partial M$ tem curvatura média constante $H_{g_{t}}$, para todo $t \in[a, b]$.

Lembre que $\rho \in \mathbb{R}$ é chamado um autovalor de Steklov do Laplaciano $\Delta_{g}$ se existe $f \in C^{\infty}(\bar{M})$, $f \neq 0$, tal que

$$
\begin{cases}\Delta_{g} f=0 & \text { em } M \\ \frac{\partial f}{\partial \eta^{g}}=\rho f & \text { sobre } \partial M .\end{cases}
$$

Definição 5.2. Seja $g \in \mathcal{M}$ tal que a curvatura escalar $R_{g}=0$ e a curvatura média do bordo $H_{g}$ é constante. Dizemos que $g$ é não degenerada se $H_{g}=0$ ou $H_{g}$ não é um autovalor de Steklov.

Nota 5.1. Do item (iv) na proposição 4.2 e fato que $d^{2} E_{\varphi_{0}}$ é Fredholm segue que $E$ satisfaz a condição local de Palais-Smale em uma vizinhança de $\varphi_{0}$, ver [Ramírez , 2014, nota 3].

Teorema 5.1. Seja $M$ uma variedade compacta orientada com bordo e $m=\operatorname{dim} M \geq 3$. Seja $[a, b] \ni t \longrightarrow g_{t} \in \mathcal{M}$ uma curva suave tal que para todo $t \in[a, b], g_{t}$ tem curvatura escalar nula e a curvatura média do bordo $\partial M, H_{g_{t}}$, é constante. Dado $t \in[a, b]$ denotaremos por $\mathfrak{n}_{t}$ o número de autovalores de Steklov (contando multiplicidade) que são menores que $H_{g_{t}}$. Suponha que

i) as métricas $g_{a}$ e $g_{b}$ são não degeneradas, 
ii) $\mathfrak{n}_{a} \neq \mathfrak{n}_{b}$.

Então existe um instante de bifurcação $\left.t_{*} \in\right] a, b\left[\right.$ para a família $\left(g_{t}\right)_{t \in[a, b]}$.

Demonstração. A prova é uma aplicação do teorema de bifurcação sobre domínio variável, ver teorema 3.3. 


\section{Capítulo 6}

\section{Bifurcação das soluções do problema de Yamabe sobre variedades produto}

Neste capítulo vamos apresentar o resultado principal desta tese. Iniciamos o capítulo relembrado alguns fatos sobre as variedades produto. Logo provamos a proposição 6.1 que desempenhará um papel importante na prova de nosso resultado principal, o teorema 6.1.

Seja $\left(M_{1}, g^{(1)}\right)$ uma variedade Riemanniana compacta orientada sem bordo e curvatura escalar nula, e seja $\left(M_{2}, g^{(2)}\right)$ uma variedade Riemanniana compacta com bordo orientada de curvatura escalar nula e curvatura média do bordo constante. Considere a variedade produto $M=M_{1} \times$ $M_{2}$, claramente $M$ é uma variedade compacta orientada com bordo dado por $\partial M=M_{1} \times \partial M_{2}$. Denotamos por $m_{i}$ a dimensão de $M_{i}, i=1,2$. Assuma que $m=\operatorname{dim} M=m_{1}+m_{2} \geq 3$. Para cada $t \in] 0, \infty\left[\right.$, denotamos por $g_{t}$ a métrica Riemanniana sobre a variedade produto $M$ definida por

$$
g_{t}=g^{(1)} \oplus t g^{(2)} .
$$

Obviamente a métrica $g_{t}$ tem curvatura escalar nula e o bordo $\partial M$ é uma subvariedade de curvatura média constante de $\left(M, g_{t}\right)$, para cada $\left.t \in\right] 0, \infty[$. Ainda mais, a curvatura média do bordo, com respeito à $g_{t}$, é dada por

$$
H_{g_{t}}=\frac{m_{2}-1}{(m-1) \sqrt{t}} H_{g^{(2)}}=\frac{\hat{H}_{g^{(2)}}}{\sqrt{t}}, \quad \text { para todo } t>0,
$$

onde $\hat{H}_{g^{(2)}}=\frac{m_{2}-1}{(m-1)} H_{g^{(2)}}$.

Temos interesse em determinar os instantes de bifurcação para a família de métricas $\left(g_{t}\right)_{t>0}$. Para isso, precisamos determinar os $t>0$ para os quais a curvatura média do bordo $H_{g_{t}}$ é um autovalor de Steklov para o Laplaciano $\Delta_{g_{t}}$. Com esta finalidade vamos caracterizar os autovalores de Steklov para o operador de Laplace $\Delta_{g_{t}}$. Sejam

$$
0=\rho^{(0)}<\rho^{(1)}<\rho^{(2)}<\rho^{(3)}<\ldots
$$

a sequência de todos os autovalores distintos de $\Delta_{g^{(1)}}, \mu^{(i)}$ a multiplicidade geométrica de $\rho^{(i)}$ e $\rho_{j}^{(i)}(t)$ o $j$-ésimo autovalor do problema

$$
\begin{cases}\Delta_{g^{(2)}} \varphi+t \rho^{(i)} \varphi=0 & \text { em } M_{2} \\ \frac{\partial \varphi}{\partial \eta^{2}}=\rho \varphi & \text { sobre } \partial M_{2}\end{cases}
$$

com $t>0$. Denotaremos por $\mu_{j}^{(i)}$ a multiplicidade geométrica de $\rho_{j}^{(i)}(t)$. Note que os $\rho_{j}^{(i)}(t)$ 's não são necessariamente distintos. Sejam $\psi^{(i)}$ e $\psi_{j}^{(i)}$ autofunções associadas aos autovalores $\rho^{(i)}$ e $\rho_{j}^{(i)}(t)$, 
respectivamente. Então

$$
\begin{aligned}
\Delta_{g_{t}} \psi^{(i)} \otimes \psi_{j}^{(i)} & =\left(\Delta_{g^{(1)}} \otimes I+\frac{1}{t} I \otimes \Delta_{g^{(2)}}\right) \psi^{(i)} \otimes \psi_{j}^{(i)} \\
& =\Delta_{g^{(1)}} \psi^{(i)} \otimes I \psi_{j}^{(i)}+\frac{1}{t} I \psi^{(i)} \otimes \Delta_{g^{(2)}} \psi_{j}^{(i)} \\
& =\rho^{(i)}\left(\psi^{(i)} \otimes \psi_{j}^{(i)}-\psi^{(i)} \otimes \psi_{j}^{(i)}\right) \\
& =0
\end{aligned}
$$

Além disso

$$
\begin{aligned}
\frac{\partial \psi^{(i)} \otimes \psi_{j}^{(i)}}{\partial \eta^{g t}} & =\left(0+\frac{1}{\sqrt{t}} \eta^{g^{(2)}}\right) \psi^{(i)} \otimes \psi_{j}^{(i)} \\
& =\psi^{(i)} \otimes \frac{1}{\sqrt{t}} \eta^{g^{(2)}} \psi_{j}^{(i)} \\
& =\psi^{(i)} \otimes \frac{1}{\sqrt{t}} \rho_{j}^{(i)}(t) \psi_{j}^{(i)} \\
& =\frac{1}{\sqrt{t}} \rho_{j}^{(i)}(t) \psi^{(i)} \otimes \psi_{j}^{(i)}
\end{aligned}
$$

Logo de (6.2) e (6.3) temos que $\frac{\rho_{j}^{(i)}(t)}{\sqrt{t}}$ é um autovalor de Steklov para o Laplaciano $\Delta_{g_{t}}$ para todo $i, j \in \mathbb{N}^{*}$. Um argumento de densidade mostra que todos os autovalores de Steklov para o operador $\Delta_{g_{t}}$ são da forma $\frac{\rho_{j}^{(i)}(t)}{\sqrt{t}}$, para algum $i, j \in \mathbb{N}^{*}$.

Nota 6.1. $\quad$ i) Note que a curvatura média do bordo $H_{g_{t}}$ é um autovalor de Steklov para $\Delta_{g_{t}}$ se e só se $\rho_{j}^{(i)}(t)=\hat{H}_{g^{(2)}}$ para algum $i, j \in \mathbb{N}^{*}$.

ii) Para $i=0$ e $j \in \mathbb{N}^{*}, \rho_{j}^{(i)}(t)$ é um autovalor de Steklov para o operador $\Delta_{g^{(2)}}$. Logo, se $\hat{H}_{g^{(2)}}$ é um autovalor de Steklov para o operador $\Delta_{g^{(2)}}$, então $H_{g_{t}}$ é um autovalor de Steklov de $\Delta_{g_{t}}$ para todo $t>0$. Assim, a fim de usar o teorema 5.1 precisamos impor a condição de que $\hat{H}_{g^{(2)}}$ não seja um autovalor de Steklov para $\Delta_{g^{(2)}}$.

Proposição 6.1. Assuma que $\hat{H}_{g^{(2)}}$ não é um autovalor de Steklov para $\Delta_{g^{(2)}}$. Para $i \in \mathbb{N}$, seja $\rho_{0}^{(i)}(t)$ o primeiro autovalor do problema (6.1). Então

i) A função $(0, \infty) \ni t \longrightarrow \rho_{0}^{(i)}(t) \in \mathbb{R}$ é contínua;

ii) $\rho_{0}^{(i)}(t) \longrightarrow 0$, quando $t \longrightarrow 0$;

iii) $\rho_{0}^{(i)}(t) \longrightarrow \infty$, quando $t \longrightarrow \infty$;

iv) Se $H_{g^{(2)}}>0$, exite só um $t_{i}>0$ tal que $\rho_{0}^{(i)}\left(t_{i}\right)=\hat{H}_{g^{(2)}}$.

Demonstração. Como a função $(0, \infty) \ni t \longrightarrow \rho_{0}^{(i)}(t) \in \mathbb{R}$ é estritamente crescente, ver proposição A.2 no apêndice A, a fim de provar (i) mostraremos que dado $t_{0}>0$ e quaisquer duas sequências $\left(t_{n}^{(1)}\right)$ e $\left(t_{n}^{(2)}\right)$ tais que $t_{n}^{(1)} \searrow t_{0}$ e $t_{n}^{(2)} \nearrow t_{0}$ temos que

$$
\inf _{n} \rho_{0}^{(i)}\left(t_{n}^{(1)}\right)=\rho_{0}^{(i)}\left(t_{0}\right)=\sup _{n} \rho_{0}^{(i)}\left(t_{n}^{(2)}\right) .
$$

Com efeito, dada a sequência $\left(t_{n}^{(1)}\right)$ tal que $t_{n}^{(1)} \searrow t_{0}$, claramente

$$
\rho_{0}^{(i)}\left(t_{0}\right)<\rho_{0}^{(i)}\left(t_{n}^{(1)}\right) \quad \text { para todo } n \in \mathbb{N} .
$$


Logo

$$
\rho_{0}^{(i)}\left(t_{0}\right) \leq \inf _{n} \rho_{0}^{(i)}\left(t_{n}^{(1)}\right) .
$$

Por outro lado, da caracterização variacional de $\rho_{0}^{(i)}\left(t_{0}\right)$, ver proposição A.1 no apêndice A, existe $\varphi \in C^{\infty}\left(\overline{M_{2}}\right)$ tal que

$$
\rho_{0}^{(i)}\left(t_{0}\right)=E_{t_{0}}(\varphi):=\int_{M_{2}}\left(g\left(\operatorname{grad}_{g^{(2)}} \varphi, \operatorname{grad}_{g^{(2)}} \varphi\right)+t_{0} \rho^{(i)} \varphi^{2}\right) v_{g^{(2)}}
$$

Logo da caracterização variacional de $\rho_{0}^{(i)}\left(t_{n}^{(1)}\right)$ segue que

$$
\rho_{0}^{(i)}\left(t_{n}^{(1)}\right) \leq E_{t_{n}^{(1)}}(\varphi)=\int_{M_{2}}\left(g\left(\operatorname{grad}_{g^{(2)}} \varphi, \operatorname{grad}_{g^{(2)}} \varphi\right)+t_{n}^{(1)} \rho^{(i)} \varphi^{2}\right) v_{g^{(2)}}, \quad \text { para todo } n \in \mathbb{N} .
$$

Portanto

$$
\inf _{n} \rho_{0}^{(i)}\left(t_{n}^{(1)}\right) \leq \inf _{n} E_{t_{n}^{(1)}}(\varphi)=E_{t_{0}}(\varphi)=\rho_{0}^{(i)}\left(t_{0}\right) .
$$

A igualdade da esquerda em (6.4) segue de (6.5) e (6.6).

Agora vamos provar a igualdade da direita em (6.4). Dada a sequência $\left(t_{n}^{(2)}\right)_{n}$ tal que $t_{n}^{(2)} \nearrow t_{0}$, é obvio que

$$
\sup _{n} \rho_{0}^{(i)}\left(t_{n}^{(2)}\right) \leq \rho_{0}^{(i)}\left(t_{0}\right)
$$

Dado $n \in \mathbb{N}$, seja $\varphi_{n} \in \mathcal{C}^{\infty}\left(\overline{M_{2}}\right)$ tal que

$$
\rho_{0}^{(i)}\left(t_{n}^{(2)}\right)=E_{t_{n}^{(2)}}\left(\varphi_{n}\right), \int_{\partial M_{2}} \varphi_{n}^{2} \sigma_{g^{(2)}}=1 \text {, and } \int_{\partial M_{2}} \varphi_{n} \sigma_{g^{(2)}}=0 .
$$

Tal $\varphi_{n}$ existe em virtude da caracterização variacional de $\rho_{0}^{(i)}\left(t_{n}^{(2)}\right)$. Note que $\left\{\varphi_{n}\right\}$ é um subconjunto limitado de $\mathrm{H}^{1}\left(M_{2}\right)$. Como subconjuntos limitados de um espaço de Hilbert são fracamente compactos, existe uma subsequência de $\left\{\varphi_{n}\right\}$, também denotada por $\left\{\varphi_{n}\right\}$, que converge fracamente a uma função $\psi \in \mathrm{H}^{1}\left(M_{2}\right)$. Portanto

$$
\int_{\partial M_{2}} \psi \sigma_{g^{(2)}}=0
$$

Como as inclusões de $\mathrm{H}^{1}\left(M_{2}\right)$ em $\mathrm{L}_{2}\left(\partial M_{2}\right)$ e $\mathrm{L}_{2}\left(M_{2}\right)$ são compactas, então a subsequência $\left\{\varphi_{n}\right\}$ satisfaz

$$
\int_{\partial M_{2}} \varphi_{n}^{2} \sigma_{g^{(2)}} \longrightarrow \int_{\partial M_{2}} \psi^{2} \sigma_{g^{(2)}} \text { e } \int_{M_{2}} \varphi_{n}^{2} \sigma_{g^{(2)}} \longrightarrow \int_{M_{2}} \psi^{2} \sigma_{g^{(2)}}
$$

Assim $\int_{\partial M_{2}} \psi^{2} \sigma_{g^{(2)}}=1$. Logo da caracterização variacional de $\rho_{0}^{(i)}\left(t_{0}\right)$ obtemos

$$
\rho_{0}^{(i)}\left(t_{0}\right) \leq E_{t_{0}}(\psi)
$$

Mas

$$
\int_{M_{2}} g\left(\operatorname{grad}_{g^{(2)}} \psi, \operatorname{grad}_{g^{(2)}} \psi\right) \sigma_{g^{(2)}} \leq \limsup \int_{M_{2}} g\left(\operatorname{grad}_{g^{(2)}} \varphi_{n}, \operatorname{grad}_{g^{(2)}} \varphi_{n}\right) \sigma_{g^{(2)}}
$$

então

$$
E_{t_{0}}(\psi) \leq \limsup _{n} E_{t_{n}^{(2)}}\left(\varphi_{n}\right)=\limsup _{n} \rho_{0}^{(i)}\left(t_{n}^{(2)}\right)=\sup _{n} \rho_{0}^{(i)}\left(t_{n}^{(2)}\right) .
$$

Combinando (6.7), (6.8) e (6.9) obtemos a igualdade do lado direito em (6.4), o qual completa a prova de (i). 
Para provar (ii), primeiro note que $0 \leq \rho_{0}^{(i)}(t)$ para todo $t>0$. Logo

$$
0 \leq \inf _{t>0} \rho_{0}^{(i)}(t)
$$

Por outro lado, do lema A.1 existe $C>0$ tal que

$$
\int_{M_{2}} \varphi^{2} v_{g^{(2)}} \leq C\left\{\int_{M_{2}} g\left(\operatorname{grad}_{g^{(2)}} \varphi, \operatorname{grad}_{g^{(2)}} \varphi\right) v_{g^{(2)}}+\int_{\partial M_{2}} \varphi^{2} \sigma_{g^{(2)}}\right\}
$$

para toda $\varphi \in \mathrm{H}^{1}\left(M_{2}\right)$. Assim,

$E_{t}(\varphi) \leq \int_{M_{2}} g\left(\operatorname{grad}_{g^{(2)}} \varphi, \operatorname{grad}_{g^{(2)}} \varphi\right) v_{g^{(2)}}+t \rho^{(i)} C\left\{\int_{M_{2}} g\left(\operatorname{grad}_{g^{(2)}} \varphi, \operatorname{grad}_{g^{(2)}} \varphi\right) v_{g^{(2)}}+\int_{\partial M_{2}} \varphi^{2} \sigma_{g^{(2)}}\right\}$.

Logo

$$
\frac{E_{t}(\varphi)}{\int_{\partial M_{2}} \varphi^{2} \sigma_{g^{(2)}}} \leq\left(1+t \rho^{i} C\right) \frac{\int_{M_{2}} g\left(\operatorname{grad}_{g^{(2)}} \varphi, \operatorname{grad}_{g^{(2)}} \varphi\right) v_{g^{(2)}}}{\int_{\partial M_{2}} \varphi^{2} \sigma_{g^{(2)}}}+t \rho^{i} C,
$$

para toda $\varphi \in \mathbb{H}^{1}\left(M_{2}\right) \backslash\{0\}$. Usando a caracterização variacional de $\rho_{0}^{(i)}(t)$ segue das equações (6.10) e (6.11) que

$$
0 \leq \rho_{0}^{(i)}(t) \leq t \rho^{(i)} C, \quad \text { para todo } t>0
$$

Portanto

$$
\rho_{0}^{(i)}(t) \longrightarrow 0 \text { quando } \quad t \longrightarrow 0 \text {. }
$$

A fim de provar (iii) suponha que a tese é falsa. Seja $\rho \in \mathbb{R}$ tal que $\lim _{t \longrightarrow \infty} \rho_{0}^{(i)}(t)=\rho$. Claramente

$$
\rho_{0}^{(i)}(t) \leq \rho \quad \text { para todo } t>0
$$

Seja $\varphi_{t} \in \mathcal{C}^{\infty}\left(\overline{M_{2}}\right)$ tal que

$$
\rho_{0}^{(i)}(t)=E_{t}\left(\varphi_{t}\right) \quad \text { e } \quad \int_{\partial M} \varphi_{t}^{2} \sigma_{g^{(2)}}=1
$$

Note que $\left\{\varphi_{t}\right\}_{t>0}$ é um subconjunto limitado de $\mathrm{H}^{1}(M)$. Afirmamos que

$$
\inf _{t>0} \int_{M_{2}} \varphi_{t}^{2} v_{g^{(2)}}>0
$$

Com efeito, caso contrário existe uma sequência $\left(\varphi_{t_{n}}\right)_{n} \subset\left\{\varphi_{t}\right\}_{t>0}$ tal que

$$
\int_{M_{2}} \varphi_{t_{n}}^{2} v_{g^{(2)}} \longrightarrow 0 \quad \text { quando } n \longrightarrow \infty
$$

Como $\left(\varphi_{t_{n}}\right)_{n}$ é limitada em $\mathrm{H}^{1}(M)$ existe uma subsequência de $\left(\varphi_{t_{n}}\right)_{n}$, que denotaremos por $\left(\varphi_{t_{n}}\right)_{n}$, tal que

$$
\int_{\partial M_{2}} \varphi_{t_{n}}^{2} \sigma_{g^{(2)}} \longrightarrow 0 \quad \text { quando } n \longrightarrow \infty
$$

Mas isto é impossível, pois

$$
\int_{\partial M_{2}} \varphi_{t_{n}}^{2} v_{g^{(2)}}=1, \quad \text { para todo } n \in \mathbb{N} .
$$

Como $\inf _{t>0} \int_{M_{2}} \varphi_{t}^{2} v_{g^{(2)}}>0$, existe $t>0$ tal que

$$
\rho<t \inf _{t>0} \int_{M_{2}} \varphi_{t}^{2} v_{g^{(2)}} \leq \rho_{0}^{(i)}(t),
$$


o que contradiz (6.13). Portanto

$$
\lim _{t \longrightarrow \infty} \rho_{0}^{(i)}(t)=\infty
$$

Finalmente, (iv) é consequência direita de (i)-(iii). Como $\left\{\varphi_{t}\right\}_{t>0}$ é um subconjunto limitado de $\mathrm{H}^{1}\left(M_{2}\right)$

Definição 6.1. Um instante $t>0$ é um instante de degeneração da família $\left(g_{t}\right)_{t>0}$ se $H_{g_{t}}$ é um autovalor de Steklov do Laplaciano $\Delta_{g_{t}}$.

Corolario 6.1. Seja $\left(M_{1}, g^{(1)}\right)$ uma variedade Riemanniana compacta orientada sem bordo e curvatura escalar nula. Seja $\left(M_{2}, g^{(2)}\right)$ uma variedade Riemanniana orientada, compacta, com bordo, curvatura escalar nula, e curvatura média do bordo constante. Denotamos por $M$ a variedade produto $M_{1} \times M_{2}$. Considere a família de métricas Riemannianas $g_{t}=g^{(2)} \oplus t g^{(2)}, t>0$, sobre $M$ e suponha que $m=\operatorname{dim} M \geq 3$. Assuma que $H_{g^{(2)}}>0$ e $\hat{H}_{g^{(2)}}$ não é um autovalor de Steklov para $\Delta_{g^{(2)}}$. Então, existe uma sequência estritamente decrescente convergindo a zero constituída por instantes de degeneração da família $\left(g_{t}\right)_{t>0}$.

Demonstração. Para cada $i \in \mathbb{N}$, seja $t_{i}$ o único número real positivo tal que $\rho_{0}^{(i)}\left(t_{i}\right)=\hat{H}_{g^{(2)}}$. Logo, para $0<i_{1}<i_{2}$ obtemos

$$
H_{g^{(2)}}=\rho_{0}^{\left(i_{1}\right)}\left(t_{i_{1}}\right)<\rho_{0}^{\left(i_{2}\right)}\left(t_{i_{1}}\right) \leq \rho_{0}^{\left(i_{2}\right)}(t), \quad t \geq t_{i_{1}} .
$$

Portanto

$$
t_{i+1}<t_{i} \quad \text { para todo } i \in \mathbb{N} \text {. }
$$

Assim, a sequência $\left(t_{i}\right)_{i \in \mathbb{N}}$ é estritamente decrescente. Claramente cada $t_{i}$ é um instante de degeneração para a família $\left(g_{t}\right)_{t>0}$. Dado $i \in \mathbb{N}$, seja $\varphi_{i} \in \mathcal{C}^{\infty}\left(\overline{M_{2}}\right)$ tal que

$$
\rho_{0}^{(i)}\left(t_{i}\right)=E_{t_{i}}\left(\varphi_{i}\right) \quad \text { e } \int_{\partial M_{2}} \varphi_{i}^{2} \sigma_{g^{(2)}}=1
$$

Como $\rho_{0}^{(i)}\left(t_{i}\right)=\hat{H}_{g^{(2)}}$ para todo $i \in \mathbb{N}$, procedendo como na prova da desigualdade (6.14), pode se provar que

$$
\inf _{i} \int_{M_{2}} \varphi_{i}^{2} v_{g^{(2)}}>0
$$

Portanto

$$
0<t_{i}=\frac{\hat{H}_{g^{(2)}}-\int_{M_{2}} g\left(\nabla \varphi_{i}, \nabla \varphi_{i}\right) v_{g^{(2)}}}{\rho^{i} \int_{M_{2}} \varphi_{i}^{2} v_{g^{(2)}}} \leq \frac{\hat{H}_{g_{(2)}}}{\rho^{i} \inf _{i} \int_{M_{2}} \varphi_{i}^{2} v_{g^{(2)}}} \longrightarrow 0,
$$

quando $i \longrightarrow \infty$. Isto prova a tese.

Estamos pronto para nosso resultado principal.

Teorema 6.1. Sejam $\left(M_{1}, g^{(1)}\right)$ uma variedade Riemanniana compacta orientada sem bordo com curvatura escalar nula e $\left(M_{2}, g^{(2)}\right)$ uma variedade Riemanniana compacta orientada com bordo, curvatura escalar zero e curvatura média do bordo constante positiva. Assuma que $\hat{H}_{g^{(2)}}$ não é um autovalor de Steklov para o Laplaciano $\Delta_{g^{(2)}}$ e $m=\operatorname{dim} M \geq 3$. Dado $\left.t \in\right] 0, \infty$ [, considere a métrica Riemanniana $g_{t}=g^{(1)} \oplus t g^{(2)}$ sobre a variedade produto $M=M_{1} \times M_{2}$. Então o conjunto de instantes de degeneração da família $\left(g_{t}\right)_{t>0}$ está constituído por uma sequência $\left(t_{i}\right)_{i \in \mathbb{N}}$ que acumula em zero. Todo instante de degeneração é um instante de bifurcação para a família $\left(g_{t}\right)_{t>0}$.

Demonstração. O corolário 6.1 estabelece a existência de uma sequência estritamente decrescente $\left(\hat{t}_{i}\right)$ convergindo a zero tal que $\hat{t}_{i}$ é um instante de degeneração da família $\left(g_{t}\right)_{t>0}$, para todo $i$. Note 
que

$$
\hat{H}_{g^{(2)}}=\rho_{0}^{(1)}\left(\hat{t}_{1}\right)<\rho_{0}^{(i)}(t) \leq \rho_{j}^{(i)}(t), \quad i, j \in \mathbb{N}, \text { e } t>\hat{t}_{1} .
$$

Afirmamos que existe no máximo um número finito de instantes de degeneração para a família $\left(g_{t}\right)_{t>0}$ no intervalo $] \hat{t}_{i+1}, \hat{t}_{i}[$. Com efeito, note que

$$
\hat{H}_{g^{(2)}}=\rho_{0}^{(i+1)}\left(\hat{t}_{i+1}\right)<\rho_{0}^{(i+1)}(t) \leq \rho_{j}^{(r)}(t), \quad r \geq i+1, t>\hat{t}_{i+1}, j \in \mathbb{N}^{*} .
$$

Assim, se $\left.\rho_{j}^{(r)}(t)=\hat{H}_{g^{(2)}}, t \in\right] \hat{t}_{i+1}, \hat{t}_{i}[$, então $1 \leq r \leq i$. Além disso, para todo $r$ existe $J(r) \in \mathbb{N}$ tal que

$$
\hat{H}_{g^{(2)}}<\rho_{j}^{(r)}\left(\hat{t}_{i+1}\right)<\rho_{j}^{(r)}(t), \quad j>J(r), t>\hat{t}_{i+1} .
$$

Seja

$$
J_{0}=\max _{1 \leq r \leq i} \min J(r) .
$$

Logo, se $\left.\rho_{j}^{(r)}(t)=\hat{H}_{g^{(2)}} \operatorname{com} t \in\right] \hat{t}_{i+1}, \hat{t}_{i}[$ então

$$
1 \leq r \leq i \quad \text { e } \quad 1 \leq j \leq J_{0} .
$$

Finalmente, para $i_{1}<i_{2}$ temos que

$$
\rho_{j}^{\left(i_{1}\right)}(t)<\rho_{j}^{\left(i_{2}\right)}(t) \quad \text { para todo } t \text { e } j .
$$

Portanto, para cada par $((r, j))$ existe no máximo um $t=t(r, j)$ tal que

$$
\rho_{j}^{(r)}(t)=\hat{H}_{g^{(2)}},
$$

o qual prova nossa afirmação. Logo, o conjunto de instantes de degeneração para a família $\left(g_{t}\right)_{t>0}$ consiste de uma sequência estritamente decrescente $\left(t_{i}\right)_{i \in \mathbb{N}}$ convergindo a zero.

Agora, para cada $i \in \mathbb{N}$ pode-se escolher $\epsilon=\epsilon(i)>0$ tal que

$$
\left[t_{i}-\epsilon, t_{i}+\epsilon\right] \cap\left(t_{i}\right)_{i \in \mathbb{N}}=\left\{t_{i}\right\} .
$$

Além disso, para todo $i$ e $j$ a função

$$
t \longrightarrow \rho_{j}^{(i)}(t)
$$

é estritamente crescente. Portanto

$$
\mathfrak{n}_{t_{i}-\epsilon} \neq \mathfrak{n}_{t_{i}+\epsilon} .
$$

O teorema 5.1 garante que todos os instantes de degeneração para a família $\left(g_{t}\right)_{t>0}$ são instantes de bifurcação. 


\section{Apêndice A}

\section{Um problema de autovalor}

Dada uma variedade compacta $M$ com bordo $\partial M \neq \emptyset$ e um número real não negativo $\lambda$, temos interesse no estudo do problema de autovalores

$$
\begin{cases}\Delta_{g} \varphi+\lambda \varphi=0, & \text { em } M, \\ \frac{\partial \varphi}{\partial \eta}=\rho \varphi, & \text { sobre } \partial M .\end{cases}
$$

Onde $g$ é uma métrica Riemanniana sobre $M, \Delta_{g}$ denota o operador Laplaciano induzido pela métrica $g$, isto é,

$$
\Delta_{g} \varphi=-\operatorname{tr}_{g}\left(\operatorname{Hess}_{\varphi}\right), \text { para toda } \varphi \in \mathcal{C}^{2}(M),
$$

e $\eta$ denota o campo normal unitário exterior ao bordo $\partial M$ com respeito à métrica $g$. No caso $\lambda=0$ o problema (A.1) é conhecido como problema de Steklov. Neste caso, Escobar em [Escobar , 2002] prova que existe uma sequência não decrescente e não limitada $\left\{\rho_{j}\right\}$ de autovalores para o problema (A.1) tal que o espaço gerado pela famlia de autofunções $\left\{\varphi_{j}\right\}$ é denso em $\mathrm{L}_{2}(M)$. Portanto, nesta seção, vamos considerar $\rho>0$ e usando as técnicas empregadas por Escobar em [Escobar , 2002] vamos caracterizar os autovalores de (A.1).

O seguinte lema será uma ferramenta muito importante nas provas desta seção.

Lema A.1. Existe uma constante positiva $C$ tal que

$$
\int_{M} \varphi^{2} v_{g} \leq C\left\{\int_{M} g\left(\operatorname{grad}_{g} \varphi, \operatorname{grad}_{g} \varphi\right) v_{g}+\int_{\partial M} \varphi^{2} \sigma_{g}\right\}
$$

para toda $\varphi \in \mathrm{H}^{1}(M)$.

Demonstração. Denotamos por

$$
\mathcal{B}(\varphi)=\int_{M} g\left(\operatorname{grad}_{g} \varphi, \operatorname{grad}_{g} \varphi\right) v_{g}+\int_{\partial M} \varphi^{2} \sigma_{g}, \quad \varphi \in \mathrm{H}^{1}(M),
$$

e seja $\beta=\inf _{\varphi \in \mathcal{C}} \mathcal{B}(\varphi)$, onde

$$
\mathcal{C}=\left\{\varphi \in \mathrm{H}^{1}(M) \mid \int_{M} \varphi^{2} v_{g}=1\right\} .
$$

Evidentemente, $0 \leq \beta$. Seja $\left\{\varphi_{j}\right\} \subset \mathcal{C}$ tal que $\mathcal{B}\left(\varphi_{j}\right) \longrightarrow \lambda$. Então $\left\{\mathcal{B}\left(\varphi_{j}\right)\right\}$ é limitado, além disso

$$
\left\|\varphi_{j}\right\|_{\mathrm{H}^{1}}^{2} \leq \mathcal{B}\left(\varphi_{j}\right)+\int_{M} \varphi^{2} v_{g}=\mathcal{B}\left(\varphi_{j}\right)+1
$$

Portanto $\left\{\varphi_{j}\right\}$ é limitada em $\mathrm{H}^{1}(M)$. Logo existe uma subsequência de $\left\{\varphi_{j}\right\}$, que denotaremos por $\left\{\varphi_{j}\right\}$, a qual converge fracamente à uma função $\varphi \in \mathrm{H}^{1}(M)$. Como a inclusão de $\mathrm{H}^{1}(M)$ em $\mathrm{L}_{2}(M)$ 
é compacta temos que

$$
\int_{M} \varphi_{j}^{2} v_{g} \longrightarrow \int_{M} \varphi^{2} v_{g}
$$

daí segue que $\varphi \in \mathcal{C}$, e por conseguinte

$$
\beta \geq \mathcal{B}(\varphi)
$$

Agora, pela convergência fraca de $\left\{\varphi_{j}\right\}$ à $\varphi$ e a desigualdade de Hölder temos

$$
\begin{aligned}
\int_{M} g\left(\operatorname{grad}_{g} \varphi, \operatorname{grad}_{g} \varphi\right) v_{g} & =\liminf \int_{M} g\left(\operatorname{grad}_{g} \varphi_{j}, \operatorname{grad}_{g} \varphi\right) v_{g} \\
& \leq \liminf \left(\int_{M} g\left(\operatorname{grad}_{g} \varphi_{j}, \operatorname{grad}_{g} \varphi_{j}\right) v_{g}\right)^{\frac{1}{2}}\left(\int_{M} g\left(\operatorname{grad}_{g} \varphi, \operatorname{grad}_{g} \varphi\right) v_{g}\right)^{\frac{1}{2}}
\end{aligned}
$$

assim,

$$
\left(\int_{M} g\left(\operatorname{grad}_{g} \varphi, \operatorname{grad}_{g} \varphi\right) v_{g}\right)^{\frac{1}{2}} \leq \liminf \left(\int_{M} g\left(\operatorname{grad}_{g} \varphi_{j}, \operatorname{grad}_{g} \varphi_{j}\right) v_{g}\right)^{\frac{1}{2}}
$$

portanto,

$$
\int_{M} g\left(\operatorname{grad}_{g} \varphi, \operatorname{grad}_{g} \varphi\right) v_{g} \leq \liminf \int_{M} g\left(\operatorname{grad}_{g} \varphi_{j}, \operatorname{grad}_{g} \varphi_{j}\right) v_{g} .
$$

Logo, da desigualdade acima e a compacidade da inclusão de $\mathrm{H}^{1}(M)$ em $\mathrm{L}_{2}(\partial M)$ segue que

$$
\mathcal{B}(\varphi) \leq \liminf \mathcal{B}\left(\varphi_{j}\right)=\beta
$$

portanto,

$$
\mathcal{B}(\varphi)=\beta
$$

Usando técnicas da regularidade elíptica se prova que $\varphi$ é suave em $M$. Agora, se $\beta=0$ então $\varphi \equiv$ cte em $M$ e $\varphi=0$ em $\partial M$. Logo $\varphi=0$ em $M$ o qual contradiz o fato que $\varphi \in \mathcal{C}$, portanto $\beta>0$. Por homogeneidade temos

$$
\int_{M} \varphi^{2} v_{g} \leq C\left\{\int_{M}\left|\operatorname{grad}_{g} \varphi\right|^{2} v_{g}+\int_{\partial M} \varphi^{2} \sigma_{g}\right\}, \quad \varphi \in \mathrm{H}^{1}(M),
$$

onde $C=\beta^{-1}$.

Observe que se $\varphi \in \mathcal{C}^{\infty}(M)$ é uma autofunção associada ao autovalor $\rho$ de (A.1) então

$$
\int_{M}\left(\left|\operatorname{grad}_{g} \varphi\right|^{2}+\lambda \varphi^{2}\right) v_{g}=\rho \int_{\partial M} \varphi^{2} \sigma_{g}
$$

portanto associamos a (A.1) o funcional

$$
E_{\lambda}(\varphi)=\int_{M}\left(\left|\operatorname{grad}_{g} \varphi\right|^{2}+\lambda \varphi^{2}\right) v_{g}, \quad \varphi \in \mathrm{H}^{1}(M)
$$

e a forma bilinear simétrica

$$
B_{\lambda}(\varphi, \phi)=\int_{M}\left(g\left(\operatorname{grad}_{g} \varphi, \operatorname{grad}_{g} \phi\right)+\lambda \varphi \phi\right) v_{g}, \quad \varphi, \phi \in \mathrm{H}^{1}(M) .
$$

Proposição A.1. Existe um minimizador do problema variacional

$$
\rho_{1}=\inf _{\varphi \in \mathrm{H}^{1}(M) \backslash\{0\}} \frac{E_{\lambda}(\varphi)}{\int_{\partial M} \varphi^{2} \sigma_{g}} .
$$

O minimizador $\varphi_{1} \in \mathcal{C}^{\infty}(\bar{M})$ satisfaz a equação (A.1) com $\rho=\rho_{1}$.

Demonstração. Primeiro observe que $0 \leq \rho_{1}$. Seja $\left\{\varphi_{j}\right\} \subset \mathrm{H}^{1}(M) \backslash\{0\}$, tal que $E_{\lambda}\left(\varphi_{j}\right) \longrightarrow \rho_{1}$. 
Podemos supor que $\int_{\partial M} \varphi_{j}^{2} \sigma_{g}=1$. Então

$$
\begin{aligned}
\left\|\varphi_{j}\right\|_{\mathrm{H}^{1}} & =\int_{M} g\left(\operatorname{grad}_{g} \varphi_{j}, \operatorname{grad}_{g} \varphi_{j}\right) v_{g}+\int_{M} \varphi_{j}^{2} v_{g} \\
& \leq E_{\lambda}\left(\varphi_{j}\right)+\int_{M} \varphi_{j}^{2} v_{g} \\
& \leq E_{\lambda}\left(\varphi_{j}\right)+C\left\{\int_{M} g\left(\operatorname{grad}_{g} \varphi_{j}, \operatorname{grad}_{g} \varphi_{j}\right) v_{g}+\int_{\partial M} \varphi_{j}^{2} \sigma_{g}\right\} \\
& \leq \tilde{C}\left(E_{\lambda}\left(\varphi_{j}\right)+1\right)
\end{aligned}
$$

onde na terceira linha usamos o lema A.1 e $\tilde{C}$ é uma constante positiva. Portanto $\left\{\varphi_{j}\right\}$ é limitada em $\mathrm{H}^{1}$, assim existe uma subsequência de $\left\{\varphi_{j}\right\}$, que denotaremos por $\left\{\varphi_{j}\right\}$, tal que $\left\{\varphi_{j}\right\}$ converge fracamente a uma função $\varphi_{1} \in \mathrm{H}^{1}$. Como a inclusão de $\mathrm{H}^{1}(M)$ em $\mathrm{L}_{2}(\partial M)$ é compacta, segue que

$$
\int_{\partial M} \varphi_{j}^{2} \sigma_{g} \longrightarrow \int_{\partial M} \varphi_{1}^{2} \sigma_{g}
$$

Logo

$$
\int_{\partial M} \varphi_{1}^{2} \sigma_{g}=1
$$

Por conseguinte

$$
\rho_{1} \leq E_{\lambda}\left(\varphi_{1}\right) .
$$

Agora, pela convergência fracae a desigualdade de Hölder, temos

$$
\begin{aligned}
\int_{M} g\left(\operatorname{grad}_{g} \varphi_{1}, \operatorname{grad}_{g} \varphi_{1}\right) v_{g} & =\liminf \int_{M} g\left(\operatorname{grad}_{g} \varphi_{j}, \operatorname{grad}_{g} \varphi_{1}\right) v_{g} \\
& \leq \liminf \left(\int_{M} g\left(\operatorname{grad}_{g} \varphi_{j}, \operatorname{grad}_{g} \varphi_{j}\right) v_{g}\right)^{\frac{1}{2}}\left(\int_{M} g\left(\operatorname{grad}_{g} \varphi_{1}, \operatorname{grad}_{g} \varphi_{1}\right) v_{g}\right)^{\frac{1}{2}} \\
& \leq\left(\int_{M} g\left(\operatorname{grad}_{g} \varphi_{1}, \operatorname{grad}_{g} \varphi_{1}\right) v_{g}\right)^{\frac{1}{2}}\left(\liminf \int_{M} g\left(\operatorname{grad}_{g} \varphi_{j}, \operatorname{grad}_{g} \varphi_{j}\right) v_{g}\right)^{\frac{1}{2}},
\end{aligned}
$$

na última desigualdade usamos o fato que a sequência $\left\{\varphi_{j}\right\}$ é limitada. Das desigualdades acima é claro que

$$
\int_{M} g\left(\operatorname{grad}_{g} \varphi_{1}, \operatorname{grad}_{g} \varphi_{1}\right) v_{g} \leq \liminf \int_{M} g\left(\operatorname{grad}_{g} \varphi_{j}, \operatorname{grad}_{g} \varphi_{j}\right) v_{g}
$$

Dado que a inclusão de $\mathrm{H}^{1}(M)$ em $\mathrm{L}_{2}(M)$ é compacta então

$$
\int_{M} \varphi_{j}^{2} v_{g} \longrightarrow \int_{M} \varphi_{1}^{2} v_{g}
$$

logo das equações (A.3) e (A.4) segue que

$$
E_{\lambda}\left(\varphi_{1}\right) \leq \liminf E_{\lambda}\left(\varphi_{j}\right)=\rho_{1} .
$$

Portanto

$$
E_{\lambda}\left(\varphi_{1}\right)=\rho_{1} .
$$

Observe que se $\rho_{1}=0$ então, $\varphi_{1} \equiv 0$ em $M$ o qual é absurdo pois, $\int_{\partial M} \varphi_{1}^{2} \sigma_{g}=1$, assim $\rho_{1}>0$.

Vamos provar agora que $\varphi_{1}$ é uma solução fraca de (A.1) com $\rho=\rho_{1}$. Seja $\varphi \in \mathrm{H}^{1}(M)$. Então, para a função $\varphi_{t}=\left(\varphi_{1}+t \varphi\right), t \in \mathbb{R}$, temos

$$
\rho_{1} \int_{\partial M} \varphi_{t}^{2} \sigma_{g} \leq E_{\lambda}\left(\varphi_{t}\right),
$$


daí, como $E\left(\varphi_{1}\right)=\rho_{1}$, obtemos

$$
\rho_{1} t \int_{\partial M} \varphi_{1} \varphi \sigma_{g}+\rho_{1} t^{2} \int_{\partial M} \varphi^{2} \sigma_{g} \leq 2 t B_{\lambda}\left(\varphi_{1}, \varphi\right)+t^{2} E(\varphi) .
$$

Logo,

$$
0 \leq t^{2}\left(E(\varphi)-\rho_{1} \int_{\partial M} \varphi^{2} \sigma_{g}\right)+2 t\left(B_{\lambda}\left(\varphi_{1}, \varphi\right)-\rho_{1} \int_{\partial M} \varphi_{1} \varphi \sigma_{g}\right),
$$

para todo $t \in \mathbb{R}$. Como

$$
E(\varphi) \geq \rho_{1} \int_{\partial M} \varphi^{2} \sigma_{g}
$$

então da desigualdade acima, segue se

$$
\int_{M}\left(g\left(\operatorname{grad}_{g} \varphi_{1}, \operatorname{grad}_{g} \varphi\right)+\lambda \varphi_{1} \varphi\right) v_{g}-\rho_{1} \int_{\partial M} \varphi_{1} \varphi \sigma_{g}=0,
$$

pois, caso contrário pode-se escolher $t$ apropriado tal que o sentido da desigualdade muda. Portanto,

$$
\int_{M}\left(g\left(\operatorname{grad}_{g} \varphi_{1}, \operatorname{grad}_{g} \varphi\right)+\lambda \varphi_{1} \varphi\right) v_{g}=\rho_{1} \int_{\partial M} \varphi_{1} \varphi \sigma_{g}
$$

para toda $\varphi \in \mathrm{H}^{1}(M)$.

Por último, a teoria da regularidade elíptica garante que $\varphi_{1} \in \mathcal{C}^{\infty}(\bar{M})$, (ver [Taylor , 2011a, exercício 3, pag. 350 ] ). Portanto $\varphi_{1}$ satisfaz (A.1), $\operatorname{com} \rho=\rho_{1}$.

Teorema A.1. Seja $(M, g)$ uma variedade Riemanniana compacta com bordo $\partial M \neq \emptyset$. Então existe uma sequência positiva $\left\{\rho_{j}\right\}$ não decrescente e não limitada de autovalores de (A.1) tal que a família $\left\{\varphi_{j}\right\}$ de autofunções é suave e o espaço linear gerado por esta família é denso em $\mathrm{L}_{2}(M)$.

Demonstração. Primeiro provamos que o espaço próprio associado á um autovalor de (A.1) é finito dimensional. De fato, dado um autovalor $\rho$ de (A.1) denotamos por $E^{\rho}$ o espaço próprio associado a $\rho$. Seja

$$
\tilde{E}^{\rho}=\left\{\psi \in \mathbb{L}^{2}(\partial M)|\psi=\varphi|_{\partial M}, \text { para alguma } \varphi \in E^{\rho}\right\} .
$$

Afirmamos que $\tilde{E}^{\rho}$ é dimensão finita. A fim de provar nossa afirmação provamos que a esfera unitária em $\tilde{E}^{\rho}$ é compacta. Com efeito, dada a sequência $\left\{\psi_{j}\right\} \subset \tilde{E}^{\rho}$ tal que $\int_{\partial M} \psi^{2} \sigma_{g}=1$, existe uma sequência $\left\{\varphi_{j}\right\} \subset E^{\rho}$ tal que $\psi_{j}=\left.\varphi_{j}\right|_{\partial M}$, para todo $j$. Observe que $\left\{\varphi_{j}\right\}$ é limitado em $\mathrm{H}^{1}(M)$, logo existe uma subsequência de $\left\{\varphi_{j}\right\}$, que denotamos por $\left\{\varphi_{j}\right\}$, a qual converge fracamente à uma função $\varphi$ em $\mathrm{H}^{1}(M)$. Logo, para toda $\phi \in \mathrm{H}^{1}(M)$

$$
\begin{aligned}
B_{\lambda}(\varphi, \phi) & =\lim _{j \longrightarrow \infty} \int_{M}\left(g\left(\operatorname{grad}_{g} \varphi_{j}, \operatorname{grad}_{g} \phi\right)+\lambda \varphi_{j} \phi\right) v_{g} \\
& =\lim _{j \longrightarrow \infty} \rho \int_{\partial M} \varphi_{j} \phi \sigma_{g} \\
& =\rho \int_{\partial M} \varphi \phi \sigma_{g}
\end{aligned}
$$

Na segunda igualdade usamos que $\varphi_{j} \in E^{\rho}$, para todo $j$. A igualdade acima garante que $\varphi \in E^{\rho}$, portanto $\psi=\left.\varphi\right|_{\partial M} \in \tilde{E}_{\lambda}$. Agora, como a inclusão de $\mathrm{H}^{1}(M)$ em $\mathrm{L}_{2}(\partial M)$ é compacta segue que

$$
\int_{\partial M} \psi_{j}^{2} \sigma_{g}=\int_{\partial M} \varphi_{j}^{2} \sigma_{g} \longrightarrow \int_{\partial M} \varphi^{2} \sigma_{g}
$$

$\operatorname{assim} \int_{\partial M} \psi^{2} \sigma_{g}=1$. Portanto, a esfera unitária em $\tilde{E}_{\lambda}$ é compacta e por conseguinte $\tilde{E}^{\rho}$ é de dimensão finita. Sejam $\varphi_{1}, \ldots, \varphi_{n} \in E^{\rho}$ tal que $\left\{\left.\varphi_{1}\right|_{\partial M}, \ldots,\left.\varphi_{n}\right|_{\partial M}\right\}$ é uma base para $\tilde{E}^{\rho}$. Então, dada $\varphi \in E^{\rho}$ existem escalares $v_{1}, \ldots, v_{n}$ tal que $\left.\varphi\right|_{\partial M}=\left.\sum_{i=1}^{m} v_{i} \varphi_{i}\right|_{\partial M}$. Observe que $\hat{\varphi}=\varphi-\sum_{i=1}^{m} v_{i} \varphi_{i}$ 
satisfaz a equação

$$
\begin{aligned}
\Delta_{g} \hat{\varphi}+\rho \hat{\varphi} & =0 \text { em } M \\
\frac{\partial \hat{\varphi}}{\partial \eta} & =0 \text { em } \partial M
\end{aligned}
$$

Logo como $\rho>0$, segue se que $\hat{\varphi}=0$, portanto $\varphi=\sum_{i=1}^{m} v_{i} \varphi_{i}$. Assim, $E^{\rho}$ é de dimensão finita. Vamos criar agora a sequência de autovalores $\left\{\rho_{j}\right\}$ de (A.1). Seja $\rho_{1}$ como na proposição A.1. Definimos $\rho_{j}, j>1$, como

$$
\rho_{j}=\inf _{\varphi \in \mathcal{G}_{j}} \frac{E_{\lambda}(\varphi)}{\int_{\partial M} \varphi^{2} \sigma_{g}}
$$

onde,

$$
\mathcal{G}_{j}=\left\{\varphi \in \mathrm{H}^{1}(M) \mid \int_{\partial M} \varphi \varphi_{r} \sigma_{g}=0, r=1, \ldots, j-1\right\}
$$

com $\varphi_{r}$ uma autofunção associada ao autovalor $\rho_{r}$, com

$$
\int_{\partial M} \varphi_{r}^{2} \sigma_{g}=1, \quad r=1, \ldots, j-1 .
$$

Observe que

$$
\rho_{j-1} \leq \rho_{j}<\infty
$$

Seja $\left\{f_{i}\right\} \subset \mathcal{G}_{j}$ tal que $E_{\lambda}\left(f_{i}\right) \longrightarrow \rho_{j}$. Podemos supor que $\int_{\partial M} f_{i}^{2} \sigma_{g}=1$. Então, como $\left\{E_{\lambda}\left(f_{i}\right)\right\}$ é limitado usando o lema A.1 pode se provar que $\left\{f_{i}\right\}$ é limitado em $\mathrm{H}^{1}(M)$, assim existe uma subsequência de $\left\{f_{i}\right\}$, que denotamos por $\left\{f_{i}\right\}$, a qual converge fracamente à uma função $\varphi_{j}$ em $\mathrm{H}^{1}(M)$. Então,

$$
\int_{\partial M} \varphi_{j} \varphi_{r} \sigma_{g}=\lim \int_{\partial M} f_{i} \varphi_{r} \sigma_{g}=0, \quad r=1, \ldots, j-1 .
$$

Assim, $\varphi_{j} \in \mathcal{G}_{j}$ portanto

$$
\rho_{j} \int_{\partial M} \varphi_{j}^{2} \sigma_{g} \leq E_{\lambda}\left(\varphi_{j}\right)
$$

Por outro lado, como a inclusão de $\mathrm{H}^{1}(M)$ em $\mathrm{L}_{2}(M)$ e $\mathrm{L}_{2}(\partial M)$ é compacta, temos

$$
\int_{M} f_{i}^{2} v_{g} \longrightarrow \int_{\partial M} \varphi_{j}^{2} v_{g}, \quad \text { e } \quad \int_{\partial M} f_{i}^{2} \sigma_{g} \longrightarrow \int_{\partial M} \varphi_{j}^{2} \sigma_{g} .
$$

Mas, $\int_{\partial M} f_{i}^{2} \sigma_{g}=1$, logo

$$
\int_{\partial M} \varphi_{j}^{2} \sigma_{g}=1
$$

Agora, como

$$
\int_{M}\left|\varphi_{j}\right|^{2} v_{g} \leq \liminf _{i} \int_{M}\left|f_{i}\right|^{2} v_{g}
$$

então

$$
\frac{E_{\lambda}\left(\varphi_{j}\right)}{\int_{\partial M} \varphi_{j}^{2} \sigma_{g}} \leq \liminf _{i} E_{\lambda}\left(f_{i}\right)=\rho_{j}
$$

portanto

$$
\rho_{j}=E_{\lambda}\left(\varphi_{j}\right) .
$$

Vamos mostrar agora que $\rho_{j}$ é um autovalor de (A.1) e $\varphi_{j}$ é uma autofunção associada à $\rho_{j}$. Seja $\varphi \in \mathcal{G}_{j}$ e considere a função

$$
\varphi_{t}=\left(\varphi_{j}+t \varphi\right), \quad t \in \mathbb{R}
$$


Observe que $\varphi_{t} \in \mathcal{G}_{j}, t \in \mathbb{R}$. Então

$$
\rho_{j} \int_{\partial M} \varphi_{t}^{2} \sigma_{g} \leq E_{\lambda}\left(\varphi_{t}\right)
$$

Daí, como

$$
E_{\lambda}\left(\varphi_{j}\right)=\rho_{j} \int_{\partial M} \varphi_{j}^{2} \sigma_{g}
$$

segue

$$
\left.2 t \rho_{j} \int_{\partial M} \varphi_{j} \varphi \sigma_{g}+t^{2} \rho_{j} \int_{\partial M} \varphi^{2} \sigma_{g}\right) \leq 2 t B_{\lambda}\left(\varphi_{j}, \varphi\right)+t^{2} E_{\lambda}(\varphi)
$$

portanto

$$
0 \leq t^{2}\left(E(f)-\rho_{j} \int_{\partial M} \varphi^{2} \sigma_{g}\right)+2 t\left(B_{\lambda}\left(\varphi_{j}, \varphi\right)-\rho_{j} \int_{\partial M} \varphi_{j} \varphi \sigma_{g}\right),
$$

para todo $t \in \mathbb{R}$. Como $\varphi \in \mathcal{G}_{j}$, então, $E(\varphi) \geq \rho_{j} \int_{\partial M} \varphi^{2} \sigma_{g}$. Além disso, $\rho_{j}>0$, assim da equação acima segue

$$
\int_{M}\left(g\left(\operatorname{grad}_{g} \varphi_{j}, \operatorname{grad}_{g} \varphi\right)+\lambda \varphi_{j} \varphi\right) v_{g}-\rho_{j} \int_{\partial M} \varphi_{j} \varphi \sigma_{g}=0
$$

isto é

$$
\int_{M}\left(g\left(\operatorname{grad}_{g} \varphi_{j}, \operatorname{grad}_{g} \varphi\right)+\lambda \varphi_{j} \varphi\right) v_{g}=\rho_{j} \int_{\partial M} \varphi_{j} \varphi \sigma_{g}
$$

para toda $\varphi \in \mathcal{G}_{j}$. Dada $\varphi \in \mathrm{H}^{1}(M)$ seja $\hat{\varphi}=\left(\varphi-\sum_{r=1}^{j-1} a_{r} \varphi_{r}\right)$, onde

$$
a_{r}=\int_{\partial M} \varphi_{j} \varphi_{r} \sigma_{g}, \quad r=1, \ldots, j-1 .
$$

Pela escolha das funções $\varphi_{r}, r=1, \ldots, j-1$, temos que

$$
\int_{\partial M} \varphi_{s} \varphi_{r} \sigma_{g}=0 \quad \text { para } \quad r, s=1, \ldots, j-1, r \neq s .
$$

$\operatorname{Logo} \hat{\varphi} \in \mathcal{G}_{j}$, assim da equação (A.8) segue

$$
\int_{M}\left(g\left(\operatorname{grad}_{g} \varphi_{j}, \operatorname{grad}_{g} \hat{\varphi}\right)+\rho \varphi_{j} \hat{\varphi}\right) v_{g}=\rho_{j} \int_{\partial M} \varphi_{j} \hat{\varphi} \sigma_{g}
$$

Como

$$
\int_{M}\left(g\left(\operatorname{grad}_{g} \varphi_{j}, \operatorname{grad}_{g} \varphi_{r}\right)+\lambda \varphi_{j} \varphi_{r}\right) v_{g}=0, r=1, \ldots, j-1,
$$

então

$$
\int_{M}\left(g\left(\operatorname{grad}_{g} \varphi_{j}, \operatorname{grad}_{g} \varphi\right)+\lambda \varphi_{j} \varphi\right) v_{g}=\rho_{j} \int_{\partial M} \varphi_{j} \varphi \sigma_{g},
$$

para toda $\varphi \in \mathrm{H}^{1}(M)$. De (A.9) usando a teoria da regularidade elíptica concluímos que $\varphi_{j} \in$ $\mathcal{C}^{\infty}(\bar{M})$ e consequentemente que $\varphi_{j}$ satisfaz (A.1) com $\rho=\rho_{j}$.

Provaremos agora que

$$
\rho_{j} \longrightarrow \infty \text { quando } j \longrightarrow \infty \text {. }
$$

Suponha que $\left\{\rho_{j}\right\}$ é limitada. Então como $\left\{\rho_{j}\right\}$ é não decrescente,

$$
\rho_{j} \longrightarrow \rho \text { quando } j \longrightarrow \infty
$$

para algum $\rho \in \mathbb{R}$. Seja $\varphi_{j}$ uma autofunção associada ao autovalor $\rho_{j}$, sem perda de generalidade podemos supor que $\int_{\partial M} \varphi_{j}^{2} \sigma_{g}=1$. Sejam $E$ o espaço linear gerado pela família de autofunções $\left\{\varphi_{j}\right\}$ 
e

$$
\tilde{E}=\left\{\psi \in \mathcal{C}^{\infty}(\partial M)|\psi=\varphi|_{\partial M}, \varphi \in E\right\} .
$$

Considere $\tilde{E}$ munido da estrutura de espaço de Hilbert com o produto interno induzido de $\mathrm{L}_{2}(\partial M)$. Afirmamos que $\tilde{E}$ é de dimensão finita. Para provar nossa afirmação mostramos que a esfera unitária em $\tilde{E}$ é compacta. Com efeito, seja $\left\{\phi_{j}\right\} \subset E$ tal que $\int_{\partial M} \phi_{j}^{2} \sigma_{g}=1$, para todo $j$. Para cada $j$ existem escalares $v_{j_{1}}, \ldots, v_{j_{r}}, r=r(j)$ e autofunções $\varphi_{j_{i}} \in\left\{\varphi_{j}\right\}, i=1, \ldots, r$ tais que

$$
\phi_{j}=\sum_{i=1}^{r} v_{j_{i}} \varphi_{j_{i}} .
$$

Como

$$
\int_{\partial M} \varphi_{j} \varphi_{k}=0, \quad j \neq k
$$

Para cada $j$ temos que

$$
\begin{cases}\Delta_{g} \phi_{j}+\lambda \phi_{j}=0, & \text { em } M, \\ \frac{\partial \phi_{j}}{\partial \eta}=\sum_{i=1}^{r} \rho_{j_{i}} v_{j_{i}} \varphi_{j_{i}} & \text { sobre } \partial M\end{cases}
$$

Logo

$$
\int_{M}\left(g\left(\operatorname{grad}_{g} \phi_{j}, \operatorname{grad}_{g} \phi_{j}\right)+\lambda \phi_{j}^{2}\right) v_{g}=\sum_{i=1}^{r} \rho_{j_{i}} v_{j_{i}}^{2} \leq \rho
$$

Portanto, $\left\{\phi_{j}\right\}$ é limitada em $\mathrm{H}^{1}(M)$. Assim existe uma subsequência de $\left\{\phi_{j}\right\}$, que denotaremos da mesma forma $\left\{\phi_{j}\right\}$, a qual converge fracamente à uma função $\phi \mathrm{em} \mathrm{H}^{1}(M)$. Como a inclusão de $\mathrm{H}^{1}(M)$ em $\mathrm{L}_{2}(\partial M)$ é compacta temos que $\phi_{j} \longrightarrow \phi$ em $\mathrm{L}_{2}(\partial M)$, logo

$$
\int_{\partial M} \phi_{j}^{2} \sigma_{g} \longrightarrow \int_{\partial M} \phi^{2} \sigma_{g}
$$

portanto

$$
\int_{\partial M} \phi^{2} \sigma_{g}=1
$$

Não é difícil provar que $\phi$ é uma autofunção associada a o autovalor $\rho$ de (A.1). Logo

$$
\left.\phi\right|_{\partial M} \in \tilde{E} .
$$

O qual prova que a esfera unitária de $\tilde{E}$ é compacta. Um argumento análogo ao usado para provar que o espaço próprio associado à um autovalor de (A.1) é de dimensão finita prova que $E$ é de dimensão finita o qual não pode ser pois, $\left\{\varphi_{j}\right\} \subset E$ e $\left\{\varphi_{j}\right\}$ é linearmente independente, portanto

$$
\lambda_{j} \longrightarrow \infty \text { quando } j \longrightarrow \infty .
$$

Finalmente, vejamos que $E=\oplus E^{j}$, onde $E^{j}$ denota o espaço próprio associado ao autovalor $\rho_{j}$ de (A.1), é denso em $\mathrm{L}_{2}(M)$. Como $E \subset \mathcal{C}^{\infty}(M)$, então $\bar{E} \subset \overline{\mathcal{C}^{\infty}(M)}=\mathrm{L}_{2}(M)$. Logo só resta provar que $\mathcal{C}^{\infty}(M) \subset \bar{E}$. Suponha que o resultado seja falso e denote por $\mathcal{B}=\bar{E} \cap \mathcal{C}^{\infty}(M)$. Defina

$$
\rho=\inf _{\varphi \in \mathcal{G}-0} \frac{E_{\lambda}(\varphi)}{\int_{\partial M} \varphi^{2} \sigma_{g}}
$$

onde

$$
\mathcal{G}=\left\{\varphi \in \mathcal{C}^{\infty}(M) \mid \int_{\partial M} \varphi \phi \sigma_{g}=0, \text { para toda } \phi \in \mathcal{B}\right\} .
$$

Pode se mostrar que $\rho$ é um autovalor de (A.1). Como $\left\{\rho_{j}\right\}$ é não limitada, existe um inteiro $j_{0}$ tal que $\rho<\rho_{j_{0}}$. No entanto, da definição de $\mathcal{G}$ segue que $\mathcal{G} \subset \mathcal{G}_{j}$, para todo $j$. Assim da caracterização 
variacional de $\rho_{j}$ temos que $\rho_{j} \leq \rho$, para todo $j$. Em particular, temos que $\rho_{j_{0}} \leq \rho$ o que é um absurdo, portanto $\mathcal{C}^{\infty}(M) \subset \bar{E}$ e assim $\mathrm{L}_{2}(M)=\mathcal{C}^{\infty}(M)$.

A seguinte propocição mostra a propriedade de minimax dos autovalores de (A.1)

Teorema A.2. O j-ésimo autovalor de (A.1) pode se caraterizar por

$$
\rho_{j}=\max _{\left\{\phi_{1}, \ldots, \phi_{j-1}\right\} \subset \mathcal{C}^{\infty}(\bar{M})}\left(\min _{\varphi \in \mathcal{G}\left(\phi_{1}, \ldots, \phi_{j-1}\right)} \frac{E_{\lambda}(\varphi)}{\int_{\partial M} \varphi^{2} \sigma_{g}}\right),
$$

onde

$$
\mathcal{G}\left(\phi_{1}, \ldots, \phi_{j-1}\right)=\left\{\varphi \in \mathcal{C}^{\infty}(\bar{M}) \backslash\{0\} \mid \int_{\partial M} \varphi \phi_{i} \sigma_{g}=0, i=1, \ldots, j-1\right\} .
$$

Demonstração. Dado $\left\{\phi_{1}, \ldots, \phi_{j-1}\right\} \subset \mathcal{C}^{\infty}(M)$, defina

$$
d\left(\phi_{1}, \ldots, \phi_{j-1}\right)=\min _{\varphi \in \mathcal{G}\left(\phi_{1}, \ldots, \phi_{j-1}\right)} \frac{E_{\lambda}(\varphi)}{\int_{\partial M} \varphi^{2} \sigma_{g}} .
$$

Seja $\varphi_{i}$ uma autofunção associada ao autovalor $\rho_{i}$ de (A.1), tal que

$$
\int_{\partial M} \varphi_{i}^{2} \sigma_{g}=1, \quad \text { para } i=1, \ldots, j-1 .
$$

Então, da caraterização variacional de $\rho_{j}$ temos que $\rho_{j}=d\left(\varphi_{1}, \ldots, \varphi_{j-1}\right)$, portanto

$$
\rho_{j} \leq \max _{\left\{\phi_{1}, \ldots, \phi_{j-1}\right\} \subset \mathcal{C}^{\infty}(M)}\left(\min _{\varphi \in \mathcal{G}\left(\phi_{1}, \ldots, \phi_{j-1}\right)} \frac{E_{\lambda}(\varphi)}{\int_{\partial M} \varphi^{2} \sigma_{g}}\right) .
$$

Afirmamos que

$$
\max _{\left\{\phi_{1}, \ldots, \phi_{j-1}\right\} \subset \mathcal{C}^{\infty}(M)} d\left(\phi_{1}, \ldots, \phi_{j-1}\right) \leq \rho_{j} .
$$

Com efeito, dado $\left\{\phi_{1}, \ldots, \phi_{j-1}\right\} \subset \mathcal{C}^{\infty}(\bar{M})$, sejam

$$
c_{i}, \quad i=1, \ldots, j,
$$

constantes tais que a função $\varphi=\sum_{i=1}^{j} c_{i} \varphi_{i}$ satisfaz

$$
\int_{\partial M} \varphi \phi_{i} \sigma_{g}=0, \quad i=1, \ldots, j-1,
$$

onde $\varphi_{i}$ é uma autofunção associada ao autovalor $\rho_{i}$ de $(\mathrm{A} .1), i=1, \ldots, j$. A existência de tais constantes segue do fato que (A.10) define um sistema linear de $j$-1-equações com $j$-variáveis. Podemos supor que

$$
\int_{\partial M} \varphi_{i}^{2} \sigma_{g}=1 \quad \text { para } i=1, \ldots, j .
$$

Ainda mais, podemos supor que

$$
\int_{\partial M} \varphi^{2} \sigma_{g}=1
$$

Como

$$
\int_{\partial M} \varphi_{i} \varphi_{r}=\delta_{i r}, \quad i, r=1, \ldots, j,
$$

então

$$
\sum_{i=1}^{j} c_{i}^{2}=1 .
$$


Dado que

$$
B_{\lambda}\left(\varphi_{i}, \varphi_{r}\right)=\rho_{i} \delta_{i r}, \quad i, r=1, \ldots, j
$$

então

$$
\frac{E_{\lambda}(\varphi)}{\int_{\partial M} \varphi^{2} \sigma_{g}}=\int_{M}\left(g\left(\operatorname{grad}_{g} \varphi, \operatorname{grad}_{g} \varphi\right)+\lambda \varphi^{2}\right) v_{g}=\sum_{i=1}^{j} c_{i}^{2} \rho_{i} \leq \rho_{j} .
$$

Assim,

$$
d\left(\phi_{1}, \ldots, \phi_{j-1}\right) \leq \rho_{j},
$$

para qualquer $\left\{\phi_{1}, \ldots, \phi_{j-1}\right\} \subset \mathcal{C}^{\infty}(\bar{M})$. O que prova a nossa afirmação. Por conseguinte

$$
\rho_{j}=\max _{\left\{\phi_{1}, \ldots, \phi_{j-1}\right\} \subset \mathcal{C}^{\infty}(M)}\left(\min _{\varphi \in \mathcal{G}\left(\phi_{1}, \ldots, \phi_{j-1}\right)} \frac{E_{\lambda}(\varphi)}{\int_{\partial M} \varphi^{2} \sigma_{g}}\right) .
$$

Finalizamos esta seção com a seguinte proposição

Proposição A.2. Sejam $0<\lambda_{1}<\lambda_{2}$. Denote por $\rho_{j}^{i}$ o $j$-ésimo autovalor de (A.1), com $\lambda=\lambda_{i}$, $i=1,2$. Então

$$
\rho_{j}^{1}<\rho_{j}^{2}, \quad j \in \mathbb{N} .
$$

Demonstração. É uma consequência direta do teorema A.2 e do fato que $E_{\lambda_{1}}(\varphi)<E_{\lambda_{2}}(\varphi)$, para toda $\varphi \in \mathrm{H}^{1}(M) \backslash\{0\}$. 
APÊNDICE A 


\section{Apêndice B}

\section{Operador Direchlet-Neumann}

Nosso proposito nesta seção é definir o operador Direchelt-Neumann e apresentar algumas de suas propriedades básicas. Com esta finalidade, iniciamos lembrando alguns fatos sobre formas bilineares em espaços de Hilbert. O desenvolvimento deste capítulo segue as ideias expostas em [Arendt e Mazzeo , 2007]. Para uma revisão dos conceitos empregados aqui referimos ao leitor a [Taylor, 2011a].

Sejam $\mathrm{H}$ e $\mathrm{V}$ espaços de Hilbert tais que $\mathrm{V}$ é incluído continua e densamente em $\mathrm{H}$. Uma forma bilinear $B: \mathrm{V} \times \mathrm{V} \longrightarrow \mathbb{R}$ simétrica e continua é dita elíptica se

$$
B(u)+w\|u\|_{\mathrm{H}}^{2} \geq \alpha\|u\|_{\mathrm{V}}^{2}, \quad u \in \mathrm{V},
$$

para alguns $w \in \mathbb{R}$ e $\alpha \geq 0$, onde $B(u)=B(u, u)$. A desigualdade acima é equivalente a dizer que a forma bilinear $B$ é limitada inferiormente e fechada em $\mathrm{H}$. Denotamos por $T_{B}$ o operador (não limitado) sobre $\mathrm{H}$ associado com $B$. Isto é, para todo $x, y \in \mathrm{H}$ temos

$$
x \in \operatorname{Dom}\left(T_{B}\right), \quad T_{B}(x)=y \Longleftrightarrow x \in \mathrm{V}, \quad B(x, u)=\langle y, u\rangle_{\mathrm{H}}, \quad \forall u \in \mathrm{V} .
$$

Segue que $T_{B}$ é um operador autoadjunto. O operador $T_{B}$ é dito monótono se

$$
\left\langle T_{B} u, u\right\rangle_{\mathrm{H}} \geq 0, \quad u \in \operatorname{Dom}\left(T_{B}\right) .
$$

Note que $T_{B}$ é monótono se e só se $B(u) \geq 0$ para todo $u \in \mathrm{V} . T_{B}$ tem resolvente compacta se e só se a inclusão $\mathrm{V} \hookrightarrow \mathrm{H}$ é compacta. Assumindo que $\mathrm{H}$ é separável e de dimensão infinita. Se $T_{B}$ tem resolvente compacta, então $\mathrm{H}$ admite uma base ortonormal $\left\{e_{n} \mid n \in \mathbb{N}\right\}$ tal que

$$
T_{B} e_{n}=\lambda_{n} e_{n}, \quad \forall n \in \mathbb{N},
$$

onde $\lambda_{1} \leq \lambda_{2} \leq \ldots$ e $\lim _{n \longrightarrow \infty} \lambda_{n}=\infty$. A sequência

$$
\lambda_{1} \leq \lambda_{2} \leq \ldots,
$$

é chamada sequência de autovalores de $T_{B}$ contando multiplicidade.

Seja $(M, g)$ uma variedade Riemanniana compacta, com bordo e dimensão $m \geq 3$. Uma função $\varphi \in \mathrm{H}^{1}(M)$ é dita harmônica se

$$
\int_{M} g\left(\operatorname{grad}_{g} \varphi, \operatorname{grad}_{g} \phi\right) v_{g}=0, \quad \forall \phi \in \mathrm{H}_{0}^{1}(M)
$$

onde

$$
\mathrm{H}_{0}^{1}(M)=\left\{\phi \in \mathrm{H}^{1}(M)|\phi|_{\partial M}=0\right\},
$$

isto é, $\mathrm{H}_{0}^{1}(M)$ denota o kernel do operador de traço $\gamma: \mathrm{H}^{1}(M) \longrightarrow \mathrm{L}_{2}(\partial M)$. Denotaremos por $\mathrm{H}_{\Delta}^{1}(M)$ o subespaço de $\mathrm{H}^{1}(M)$ das funções harmônicas. Uma função $\varphi \in \mathrm{H}_{\Delta}^{1}(M)$ tem derivada 
normal exterior em $\mathrm{L}_{2}(\partial M)$ se existe $f \in \mathrm{L}_{2}(\partial M)$ tal que

$$
\int_{M} g\left(\operatorname{grad}_{g} \varphi, \operatorname{grad}_{g} \phi\right) v_{g}=\int_{\partial M} f \phi \sigma_{g}, \quad \forall \phi \in \mathrm{H}^{1}(M) .
$$

Pode se provar que se $\varphi \in \mathrm{H}_{\Delta}^{1}(M)$ tem uma derivada normal $f$ em $\mathrm{L}_{2}(\partial M)$, esta é única, neste caso denotamos por $\frac{\partial \varphi}{\partial \eta^{g}}=f$. Seja

O operador Direchelt-Neumann $\mathcal{N}_{g}$ é definido como um operador não limitado sobre $\mathrm{L}_{2}(\partial M)$.

$$
\operatorname{Dom}\left(\mathcal{N}_{g}\right)=\left\{f \in \mathrm{L}_{2}(\partial M) \mid \exists \varphi \in \mathrm{H}_{\Delta}^{1}(M) \quad \operatorname{com} \quad \frac{\partial \varphi}{\partial \eta^{g}} \in \mathrm{L}_{2}(\partial M) \quad \text { tal que }\left.\quad \varphi\right|_{\partial M}=f\right\} .
$$

Definimos $\mathcal{N}_{g}: \mathrm{L}_{2}(\partial M) \longrightarrow \mathrm{L}_{2}(\partial M)$ por

$$
\mathcal{N}_{g}(f)=\frac{\partial \varphi}{\partial \eta^{g}}, \quad f \in \operatorname{Dom}\left(\mathcal{N}_{g}\right)
$$

onde $\varphi \in \mathrm{H}_{\Delta}^{1}(M)$ é tal que $\left.\varphi\right|_{\partial M}=f$. A boa definição de $\mathcal{N}_{g}$ é garantida pela seguinte descomposição do espaço $\mathrm{H}^{1}(M)$

$$
\mathrm{H}^{1}(M)=\mathrm{H}_{0}^{1}(M) \oplus \mathrm{H}_{\Delta}^{1}(M) .
$$

Com efeito, se $\varphi_{1}, \varphi_{2} \in \mathrm{H}_{\Delta}^{1}(M)$ são tais que $\varphi_{1}=f=\varphi_{2}$, então $\varphi_{1}-\varphi_{2} \in \mathrm{H}_{0}^{1}(M) \cap \mathrm{H}_{\Delta}^{1}(M)$, $\operatorname{logo}$ $\varphi_{1}=\varphi_{2}$. Agora, a descoposição (B.1) é consequência do seguinte fato:

Proposição B.1. Para toda $\psi \in \mathrm{H}^{1}(M)$, existe $\varphi \in \mathrm{H}_{\Delta}^{1}(M)$ tal que $\left.\psi\right|_{\partial M}=\left.\varphi\right|_{\partial M}$.

Demonstração. Ver [Taylor, 2011a, Proposição 1.7, pag. 360]

Observação B.1. 1. Da definição de $\mathcal{N}_{g}$ temos que

$$
\int_{M} g\left(\operatorname{grad}_{g} \varphi, \operatorname{grad}_{g} \phi\right) v_{g}=\int_{\partial M} \mathcal{N}_{g}(f) \phi \sigma_{g}, \quad \phi \in \mathrm{H}^{1}(M),
$$

onde $\varphi \in \mathrm{H}_{\Delta}^{1}(M) \operatorname{com} \gamma(\varphi)=f$. Em particular, para $\phi=\mathbf{1}$, temos

$$
\int_{\partial M} \mathcal{N}_{g}(f) \sigma_{g}=0, \quad f \in \operatorname{Dom}\left(\mathcal{N}_{g}\right) .
$$

2. Se $\mathcal{N}_{g}(f)=0$, então para $\varphi \in \mathrm{H}_{\Delta}^{1}(M) \operatorname{com} \gamma(\varphi)=f$ temos

$$
\int_{M} g\left(\operatorname{grad}_{g} \varphi, \operatorname{grad}_{g} \phi\right) v_{g}=0, \quad \phi \in \mathrm{H}^{1}(M) .
$$

A equação acima garante que $\varphi$ é constante, portanto $f$ é constante. O reciproco também é certo. Assim,

$$
\operatorname{Ker}\left(\mathcal{N}_{g}\right)=\left\{f \in \mathrm{L}_{2}(\partial M) \mid f=\text { constante }\right\} .
$$

Nosso objetivo agora é provar que $\mathcal{N}_{g}$ é autoadjunto, limitado inferiormente e tem resolvente compacta. Com esta finalidade, mostraremos que $\mathcal{N}_{g}$ é o operador associado a uma forma bilinear, simétrica, continua e elíptica.

Seja $V$ o espaço traço, isto é,

$$
\mathrm{V}=\left\{\left.\varphi\right|_{\partial M} \mid \varphi \in \mathrm{H}^{1}(M)\right\} .
$$

Claramente $V \subset \mathrm{L}_{2}(\partial M)$. De (B.1) segue que o operador traço restrito a $\mathrm{H}_{\Delta}^{1}(M)$, isto é, a função 
linear $\left.\gamma\right|_{\mathrm{H}_{\Delta}^{1}(M)}: \mathrm{H}_{\Delta}^{1}(M) \longrightarrow \mathrm{L}_{2}(\partial M)$ definida por

$$
\left.\gamma\right|_{H_{\Delta}^{1}(M)}(\varphi)=\left.\varphi\right|_{\partial M}
$$

es bijectiva. Para $f, g \in \mathrm{V}$, definimos

$$
\langle f, h\rangle_{\mathrm{V}}=\langle\varphi, \phi\rangle_{\mathrm{H}^{1}(M)},
$$

onde $\left.\gamma\right|_{H_{\Delta}^{1}(M)}(\varphi)=f$ e $\left.\gamma\right|_{H_{\Delta}^{1}(M)}(\phi)=h$. Note que (B.2) define um produto interno em $\mathrm{V}$ com o qual $\vee$ é um espaço de Hilbert. Como o operador traço $\gamma: \mathrm{H}^{1}(M) \longrightarrow \mathrm{L}_{2}(\partial M)$ é compacto, então a inclusão $\mathrm{V} \hookrightarrow \mathrm{L}_{2}(\partial M)$ é compacta. Finalmente o teorema de Stone-Weierstrass implica que $\mathrm{V}$ é denso em $\mathrm{L}_{2}(\partial M)$.

Seja $B: \mathrm{V} \times \mathrm{V} \longrightarrow \mathbb{R}$ definida por

$$
B(f, h)=\int_{\partial M} g\left(\operatorname{grad}_{g} \varphi, \operatorname{grad}_{g} \phi\right) v_{g}
$$

onde $\left.\gamma\right|_{H_{\Delta}^{1}(M)}(\varphi)=f$ e $\left.\gamma\right|_{H_{\Delta}^{1}(M)}(\phi)=h$. É claro que $B$ é uma forma bilinear simétrica. Além disso, a desigualdade de Hölder implica que

$$
|B(f, h)| \leq C\|\varphi\|_{\mathrm{H}^{1}(M)}\|\phi\|_{\mathrm{H}^{1}(M)}=C\|f\|_{\mathrm{V}}\|h\|_{\mathrm{V}},
$$

para alguma constante positiva $C$. Portanto $B$ é continua.

Proposição B.2. $B$ é uma forma bilinear simétrica, continua e elípica. Além disso, operador Direchlet-Neumann $\mathcal{N}_{g}$ é o operador (não limitado) sobre $\mathrm{L}_{2}(\partial M)$ associado com $B$.

Demonstração. Sejam $X_{1}=\mathrm{V}, X_{2}=\mathrm{L}_{2}(\partial M), X_{3}=\mathrm{L}_{2}(M), T: X_{1} \longrightarrow X_{3}$ e $S: X_{1} \longrightarrow X_{2}$ definidos por

$$
T(f)=\varphi \quad \text { e } \quad S(f)=f, \quad f \in X_{1},
$$

onde $\varphi \in \mathrm{H}_{\Delta}^{1}(M)$ atende $\left.\varphi\right|_{\partial M}=f$. Temos que $S$ é injetivo e $T$ é compacto, pois a inclusão $\mathrm{H}^{1}(M) \hookrightarrow \mathrm{L}_{2}(M)$ é compacta. Logo do lema B.1 temos que existe $w>0$ tal que

$$
\int_{M} \varphi^{2} v_{g} \leq \frac{1}{2}\|\varphi\|_{\mathrm{H}^{1}(M)}^{2}+w \int_{\partial M} f^{2} \sigma_{g}
$$

Isto é,

$$
w \int_{\partial M} f^{2} \sigma_{g} \geq-\frac{1}{2} \int_{M} g\left(\operatorname{grad}_{g} \varphi, \operatorname{grad}_{g} \varphi\right) v_{g}+\frac{1}{2} \int_{M} \varphi^{2} v_{g}
$$

Assim,

$$
\begin{aligned}
B(f, f)+w\|f\|_{\mathrm{L}_{2}(\partial M)}^{2} & =\int_{M} g\left(\operatorname{grad}_{g} \varphi, \operatorname{grad}_{g} \varphi\right) v_{g}+w \int_{\partial M} f^{2} \sigma_{g} \\
& \geq \frac{1}{2}\left(\int_{M} g\left(\operatorname{grad}_{g} \varphi, \operatorname{grad}_{g} \varphi\right)+v_{g}+\int_{M} \varphi^{2} v_{g}\right) \\
& =\frac{1}{2}\|f\|_{\mathbf{V}}^{2} .
\end{aligned}
$$

A desigualdade acima prova que $B$ é elíptica. Denotemos por $T_{B}$ o operador (não limitado) sobre $\mathrm{L}_{2}(\partial M)$ associado com $B$. Dada $f \in \mathrm{L}_{2}(\partial M)$ temos que $f \in \operatorname{Dom}\left(T_{B}\right)$ se e só se $f \in \mathrm{V}$ e

$$
\int_{M} g\left(\operatorname{grad}_{g} \varphi, \operatorname{grad}_{g} \phi\right) v_{g}=\int_{\partial M} T_{B}(f) \phi \sigma_{g}, \quad \forall \phi \in \mathrm{H}_{\Delta}^{1}(M),
$$


onde $\varphi \in \mathrm{H}_{\Delta}^{1}(M)$ atende $f=\left.\gamma\right|_{\mathrm{H}_{\Delta}^{1}(M)}(\varphi)$. Como

$$
\int_{M} g\left(\operatorname{grad}_{g} \varphi, \operatorname{grad}_{g} \psi\right) v_{g}=0, \quad \forall \psi \in \mathrm{H}_{0}^{1}(M)
$$

então de (B.1), (B.3) e (B.4) temos que

$$
\int_{M} g\left(\operatorname{grad}_{g} \varphi, \operatorname{grad}_{g} \phi\right) v_{g}=\int_{\partial M} T_{B}(f) \phi \sigma_{g}
$$

para toda $\phi \in \mathrm{H}^{1}(M)$. Logo, $\varphi \in \mathrm{H}_{\Delta}^{1}(M)$ tem derivada normal em $\mathrm{L}_{2}(\partial M)$ e portanto $f \in \operatorname{Dom}\left(\mathcal{N}_{g}\right)$. Além disso,

$$
\mathcal{N}_{g}(f)=\frac{\partial \varphi}{\partial \eta^{g}}=T_{B}(f) .
$$

Por outro lado, dada $f \in \operatorname{Dom}\left(\mathcal{N}_{g}\right)$, exite $\varphi \in \mathrm{H}_{\Delta}^{1}(M)$ com $\frac{\partial \varphi}{\partial \eta^{g}} \in \mathrm{L}_{2}(\partial M)$ tal que $f=$ $\left.\gamma\right|_{\mathrm{H}_{\Delta}^{1}(M)}(\varphi)$. Logo $f \in \mathrm{V}$, além disso

$$
\int_{M} g\left(\operatorname{grad}_{g} \varphi, \operatorname{grad}_{g} \phi\right) v_{g}=\int_{\partial M} \frac{\partial \varphi}{\partial \eta^{g}} \phi \sigma_{g},
$$

para toda $\phi \in \mathrm{H}^{1}(M)$. A igualdade acima implica que

$$
B(f, h)=\int_{\partial M} \frac{\partial \varphi}{\partial \eta^{g}} h \sigma_{g}
$$

para toda $h \in \mathrm{V}$. Portanto $f \in \operatorname{Dom}\left(T_{B}\right)$ e

$$
T_{B}(f)=\frac{\partial \varphi}{\partial \eta^{g}}=\mathcal{N}_{g}(f) .
$$

Corolario B.1. O operador Direchlet-Neumann $\mathcal{N}_{g}$ é autoadjunto, limitado inferiormente e tem resolvente compacta.

Lema B.1. Sejam $\mathrm{X}_{1}, \mathrm{X}_{2}, \mathrm{X}_{3}$ espaços de Banach, $\mathrm{X}_{1}$ reflexivo. Sejam $T \in \mathcal{L}\left(\mathrm{X}_{1}, \mathrm{X}_{3}\right)$ compacto e $S \in \mathcal{L}\left(\mathrm{X}_{1}, \mathrm{X}_{2}\right)$ injetivo. Dado $\epsilon>0$, existe $w>0$ tal que para todo $x \in \mathrm{X}_{1}$,

$$
\|T x\|_{\mathrm{X}_{3}}^{2} \leq \epsilon\|x\|_{\mathrm{X}_{1}}^{2}+w \mid S x \|_{\mathrm{X}_{2}}^{2}
$$

Demonstração. Caso contrario, para cada $n \in \mathbb{N}$, existe $x_{n} \in \mathrm{X}_{1}$ tal que $\left\|x_{n}\right\|=1 \mathrm{e}$

$$
\left\|T x_{n}\right\|_{\mathrm{X}_{3}}^{2} \geq \epsilon+n\left\|S x_{n}\right\|_{\mathrm{X}_{2}}^{2}
$$

Dado que $\mathrm{X}_{1}$ é reflexivo podemos supor que $x_{n} \rightarrow x$ em $\mathrm{X}_{1}$. Logo, pela compacidade de $T$, passando a subsequência se é necessário, temos

$$
T x_{n} \longrightarrow T x, \quad \text { em } \quad \mathrm{X}_{3} .
$$

Portanto

$$
\|T x\|_{\mathrm{X}_{3}}^{2} \geq \epsilon .
$$

Por outro lado,

$$
\left\|S x_{n}\right\|_{\mathrm{X}_{2}}^{2} \leq \frac{1}{n}\left\|T x_{n}\right\|_{\mathrm{X}_{3}} \longrightarrow 0 \quad \text { quando } \quad n \longrightarrow \infty .
$$

Como $x_{n} \rightarrow x$, então $S x=0$. Como $S$ é injetivo, segue que $x=0$. Portanto $T x=0$, o qual é absurdo. 
Culminamos este capítulo com a seguinte propriedade do operador $\mathcal{N}_{g}$. Proposição B.3. $\mathcal{N}_{g}$ é um operador de Fredhlom de índice zero.

Demonstração. Ver [Taylor, 2011b, pag. 44] 
APÊNDICE B 


\section{Referências Bibliográficas}

Abramovich e Aliprantis (2002) Y. A. Abramovich e C. D. Aliprantis. An invitation to operator theory. American mathematical society. Citado na pág. 5

Agmon et al. (1959) A. Agmon, A. Douglis e L. Nirenberg. Estimates near the boundary for solutions of elliptic partial differential equations satisfying general boundary conditions. I. Communications on pure and applied mathematics, XII:623-727. Citado na pág. 29, 35, 38

Almaraz (2010) S. Almaraz. An existence theorem of conformal scalar-flat metrics on manifolds with boundary. Pacific. J. Math., 248 (1):1-22. Citado na pág. 3, 29

Ambrosetti e Prodi (1993) A. Ambrosetti e G. Prodi. A primer of nonlinear analysis. Cambridge University Press. Citado na pág. 5, 23

Arendt e Mazzeo (2007) W. Arendt e R. Mazzeo. Spectral properties of the direchlet-to-neumann operator on lipschitz domains. Ulmer Seminare, Heft 12:28-38. Citado na pág. 57

Aubin (1976) T. Aubin. équations différentielles non linéaires et problème de Yamabe concernant la courbure scalaire. J. Math. Pures Appl., 55 (9):269-296. Citado na pág. 1

Aubin (1998) T. Aubin. Some nonlinear problems in Riemannian geometry. Springer. Citado na pág. 29, 35

Besse (2008) A. L. Besse. Einstein Manifolds. Springer. Citado na pág. 5

Brendle (2008) S. Brendle. Blow-up phenomena for the Yamabe equation. J. Amer. Math. Soc., 21:951-979. Citado na pág. 2

Brezis (2011) H. Brezis. Fuctional analysis, Sobolev spaces and partial differential equations. Springer, first ed. Citado na pág. 5

Carmo (2005) M. Do Carmo. Geometria Riemanniana. Projeto Euclides, terceira ed. Citado na pág. 5

Cherrier (1984) P. Cherrier. Problèmes de Neumann non linéaires sur les variétés riemanniennes. J. Funct. Anal, 57:154-206. Citado na pág. 2

Clarke (2010) B. Clarke. The metric geometry of the manifold of Riemannian metrics over a closed manifold. Calculus of variations, 39:533-545. Citado na pág. 35

Druet et al. (2004) O. Druet, E. Hebey e F. Robert. Blow-up Theory for Elliptic PDEs in Riemannian Geometry (MN-45). Princeton university press. Citado na pág. 5

Escobar (1992a) J. F. Escobar. The Yamabe problem on manifolds with boundary. J. Diff. Geom., 35:21-84. Citado na pág. 2

Escobar (1992b) J. F. Escobar. Conformal deformation of a riemannian metric to a scalar flat metric with constant mean curvature on the boundary. Annals of Math., 136:1-50. Citado na pág. $2,3,29$ 
Escobar (1996) J. F. Escobar. Conformal deformation of a riemannian metric to a constant scalar curvature metric with constant mean curvature on the boundary. Indiana Univ. Math. J., 45: 917-943. Citado na pág. 2

Escobar (2003) J. F. Escobar. Uniqueness and non-uniqueness of metrics with prescribed scalar and mean curvature on compact manifolds with boundary. Journal of functional analysis, 202: 424-442. Citado na pág. 3

Escobar (2002) J. F. Escobar. Topics in PDF's and differential geometry. Goiânia:Ed. da UFG. Citado na pág. 29, 35, 47

García e Munoz (2012) G. García e J. Munoz. On the non-uniqueness of conformal metrics with prescribed scalar and mean curvatures on compact manifolds with boundary. Revista de la unión matemática Argentina, 53(1):29-42. Citado na pág. 3

Han e Li (1999) Z. Han e Y. Li. The Yamabe problem on manifolds with boundary: existence and compactness results. Duke Math. J., 99(3):489-542. Citado na pág. 2

Hebey (2000) E. Hebey. Nonlinear analysis on manifolds: Sobolev spaces and inequalities. AMS. Citado na pág. 5

Hebey e Vaugon (1992) E. Hebey e M. Vaugon. Meilleures constantes dans le theoreme dinclusion de Sobolev et multiplicite pour les problemes de Nirenberg et Yamabe,, volume 41. Indiana Univ. Math. J. Citado na pág. 2

Hilbert (1915) D. Hilbert. Die grundlagen der physik. Nach. Ges. wiss., páginas 461-472. Citado na pág. 1

Kesavan (2003) S. Kesavan. Topics in functional analysis and applications. New age international (P) Ltd. Citado na pág. 5

Khuri et al. (2009) M Khuri, F. Marques e R. Schoen. A compactness theorem for the Yamabe problem. J. Differential Geom., 81(1):143-196. Citado na pág. 2

Kielhöfer (2004) H. Kielhöfer. Bifurcation theory. An introduction with applications to PDEs. Springer. Citado na pág. 23

Lee (1997) J. M. Lee. Riemannian Manifolds. An introduction to curvature. Springer. Citado na pág. 5

Leoni (2009) G. Leoni. A first course in Sobolev spaces. American mathematical society. Citado na pág. 5

Lima et al. (2012) L. L. De Lima, P. Piccione e M. Zedda. On bifurcation of solutions of the Yamabe problem in product manifolds. Annales de L'Institut Henri Poincare (C) Non Lin. Anal., 29:261-277. Citado na pág. 2, 3, 21, 23, 26, 29, 35

Lions e Magenes (1972) J.L. Lions e E. Magenes. Non-Homogeneous boundary value problems and applications, volume I. Springer-Verlag. Citado na pág. 5, 19, 35

Marques (2005) F. Marques. Existence results for the Yamabe problem on manifolds with boundary. Indiana Univ. Math. J., 54 (6):1599-1620. Citado na pág. 2, 3, 29

Marques (2007) F. Marques. Conformal deformation to scalar flat metrics with constant mean curvature on the boundary. Comm. Anal. Geom., 15 (2):381-405. Citado na pág. 2, 3, 29

Obata (1971) M. Obata. The conjetures on conformal transformations of Riemannian manifolds. J. Diff. Geom., 6:247-258. Citado na pág. 2 
Petean (2010) J. Petean. Metrics of constant scalar curvature conformal to riemannian products. Proc. Amer. Math. Soc., 138:2897-2905. Citado na pág. 2

Petersen (1998) P. Petersen. Riemannian Geometry. Springer, first ed. Citado na pág. 5

Pollack (1993) D. Pollack. Nonuniquenes and high energy solutions for a conformally invariant scalar equation. Comm. Anal. Geom., 1:347-414. Citado na pág. 2

Ramírez (2014) H.F. Ramírez. Multiplicity of constant scalar curvature metrics in $\mathbb{T}^{k} \times M$. Nonlinear Analysis: TMA, 109:103-112. Citado na pág. 33, 39

Schoen (1989) R. Schoen. Variational theory for the total scalar curvature functional for riemannian metrics and related topics. Lectures notes in math., 1365:120-154. Citado na pág. 2

Schoen (1984) R. Schoen. Conformal deformation of a riemannian metric to constant scalar curvature. J. Diff. Geom., 20:479-495. Citado na pág. 1

Smoller e Wasserman (1990) J. Smoller e A.G. Wasserman. Bifurcation and symmetry breaking. Invent. Math, 100:63-95. Citado na pág. 23, 26

Taylor (2011a) M. Taylor. Partial Differential Equations I: Basic Theory. Springer, second ed. Citado na pág. 5, 19, 29, 35, 50, 57, 58

Taylor (2011b) M. Taylor. Partial Differential Equations II:Qualitative estudies of linear equations. Springer, second ed. Citado na pág. 32, 35, 61

Trudinger (1968) N. Trudinger. Remarks concerning the conformal deformation of riemannian structures on compact manifolds. Annali Scuola Norm. Sup. Pisa, (3) 22:265-274. Citado na pág. 1

Yamabe (1960) H. Yamabe. On a deformation of riemannian structures on compact manifolds. Osaka Math J., 12:21-37. Citado na pág. 1 